University of Tennessee Health Science Center UTHSC Digital Commons

\title{
Understanding Human Astrovirus from Pathogenesis to Treatment
}

Virginia Hargest

University of Tennessee Health Science Center

Follow this and additional works at: https://dc.uthsc.edu/dissertations

Part of the Diseases Commons, Medical Sciences Commons, and the Viruses Commons

\section{Recommended Citation}

Hargest, Virginia (0000-0003-3883-1232), "Understanding Human Astrovirus from Pathogenesis to Treatment" (2020). Theses and Dissertations (ETD). Paper 523. http://dx.doi.org/10.21007/ etd.cghs.2020.0507.

This Dissertation is brought to you for free and open access by the College of Graduate Health Sciences at UTHSC Digital Commons. It has been accepted for inclusion in Theses and Dissertations (ETD) by an authorized administrator of UTHSC Digital Commons. For more information, please contact jwelch30@uthsc.edu. 


\title{
Understanding Human Astrovirus from Pathogenesis to Treatment
}

\begin{abstract}
While human astroviruses (HAstV) were discovered nearly 45 years ago, these small positive-sense RNA viruses remain critically understudied. These studies provide fundamental new research on astrovirus pathogenesis and disruption of the gut epithelium by induction of epithelial-mesenchymal transition (EMT) following astrovirus infection. Here we characterize HAstV-induced EMT as an upregulation of SNAI1 and VIM with a down regulation of CDH1 and OCLN, loss of cell-cell junctions most notably at 18 hours post-infection (hpi), and loss of cellular polarity by $24 \mathrm{hpi}$. While active transforming growth factor-i] (TGF-[i]) increases during HAstV infection, inhibition of TGF-[i] signaling does not hinder EMT induction. However, HAstV-induced EMT does require active viral replication. These are among the first studies describing the induction of EMT by a non-oncogenic virus and provides an exciting opportunity to understand EMT induction independent of cancer. Our findings likely extend beyond astrovirus to other viruses and may shed light on novel ways pathogens can circumvent the barriers meant to protect against them. Crossing these barriers can lead to systemic and even fatal infections. Astroviruses can be especially problematic in immunocompromised individuals and infants where the virus has been associated with necrotizing enterocolitis, severe and persistent diarrhea, and even encephalitis and meningitis. Using our novel tools and models, we demonstrate that the FDA-approved broad-spectrum anti-infective drug nitazoxanide (NTZ) blocks astrovirus replication in vitro with a $50 \%$ effective concentration (EC50) of approximately $1.47 \mu \mathrm{M}$. It can be administered up to 8 hours post-infection and is effective against multiple human astrovirus serotypes including clinical isolates. Most importantly, NTZ reduces viral shed in vivo, exhibiting its potential as a future clinical therapeutic. Overall, these studies will further our understanding of astrovirus pathogenesis leading to the development of therapeutic options for vulnerable populations.
\end{abstract}

Document Type

Dissertation

Degree Name

Doctor of Philosophy (PhD)

Program

Biomedical Sciences

Research Advisor

Stacey Schultz-Cherry, PhD

Keywords

Cellular biology, virology, microbiology

Subject Categories

Diseases | Medical Sciences | Medicine and Health Sciences | Viruses 
DOCTOR OF PHILOSOPHY DISSERTATION

\section{Understanding Human Astrovirus from Pathogenesis to Treatment}

Author:

Virginia Hargest
Advisor:

Stacey Schultz-Cherry, PhD

A Dissertation Presented for The Graduate Studies Council of

The University of Tennessee Health Science Center

in Partial Fulfillment of the Requirements for the

Doctor of Philosophy degree from

The University of Tennessee

in

Biomedical Sciences: Microbiology, Immunology, and Biochemistry

College of Graduate Health Sciences

June 2020 
Chapters 3 and 5 (C) 2020 by American Society for Microbiology. All other material (c) 2020 by Virginia Hargest.

All rights reserved. 


\section{DEDICATION}

To my amazing fiancé Winter, my Mom and Dad, and Jessie, for their constant support and encouragement. I love you and I could not have gotten here without you. 


\section{ACKNOWLEDGEMENTS}

First and foremost, I want to thank my mentor Dr. Stacey Schultz-Cherry. Stacey has allowed me to grow as a scientist and encouraged me along the way. She has taught me how to think critically, how to perform the right tests to answer the questions my project has raised, and has made me a more confident scientist. She gave me enough freedom to study what I was curious about, but reigned me back in when I would get stuck down a rabbit hole. Stacey has been there for the lows of multiple hypotheses being proven wrong and the highs of my first first-author publication and all the times in between. She is my role model for what a strong woman in science should be and I owe her everything for the scientist I have become.

I also would like to thank my committee members Dr. Mike Whitt, Dr. RK Rao, Dr. Jason Rosch, and especially Dr. Carolyn Coyne, for serving as an external member. I appreciate their time, commitment, and for their helpful guidance through my graduate career.

Finally, as every graduate student can attest, a majority of what you learn during your graduate studies comes from those you interact with on a daily basis. With that said, I want to thank the many members, past and present, of the Schultz-Cherry Lab. I want to especially thank Victoria Meliopoulos, Pam Freiden, Val Cortez, Bekah Honce, and Cydney Johnson. Whether it be teaching me a new technique, answering questions, helping with figure design, editing manuscripts, or just keeping me (mostly) sane, each of them has contributed to my success and I thank them for it. 


\begin{abstract}
While human astroviruses (HAstV) were discovered nearly 45 years ago, these small positive-sense RNA viruses remain critically understudied. These studies provide fundamental new research on astrovirus pathogenesis and disruption of the gut epithelium by induction of epithelial-mesenchymal transition (EMT) following astrovirus infection. Here we characterize HAstV-induced EMT as an upregulation of SNAII and VIM with a down regulation of $C D H 1$ and $O C L N$, loss of cell-cell junctions most notably at 18 hours post-infection (hpi), and loss of cellular polarity by $24 \mathrm{hpi}$. While active transforming growth factor- $\beta$ (TGF- $\beta$ ) increases during HAstV infection, inhibition of TGF- $\beta$ signaling does not hinder EMT induction. However, HAstV-induced EMT does require active viral replication. These are among the first studies describing the induction of EMT by a non-oncogenic virus and provides an exciting opportunity to understand EMT induction independent of cancer. Our findings likely extend beyond astrovirus to other viruses and may shed light on novel ways pathogens can circumvent the barriers meant to protect against them. Crossing these barriers can lead to systemic and even fatal infections. Astroviruses can be especially problematic in immunocompromised individuals and infants where the virus has been associated with necrotizing enterocolitis, severe and persistent diarrhea, and even encephalitis and meningitis. Using our novel tools and models, we demonstrate that the FDA-approved broad-spectrum anti-infective drug nitazoxanide (NTZ) blocks astrovirus replication in vitro with a 50\% effective concentration $\left(\mathrm{EC}_{50}\right)$ of approximately $1.47 \mu \mathrm{M}$. It can be administered up to 8 hours postinfection and is effective against multiple human astrovirus serotypes including clinical isolates. Most importantly, NTZ reduces viral shed in vivo, exhibiting its potential as a future clinical therapeutic. Overall, these studies will further our understanding of astrovirus pathogenesis leading to the development of therapeutic options for vulnerable populations.
\end{abstract}




\section{TABLE OF CONTENTS}

\section{CHAPTER 1. ASTROVIRUS VIROLOGY, PATHOGENESIS, AND DISEASE .....1}

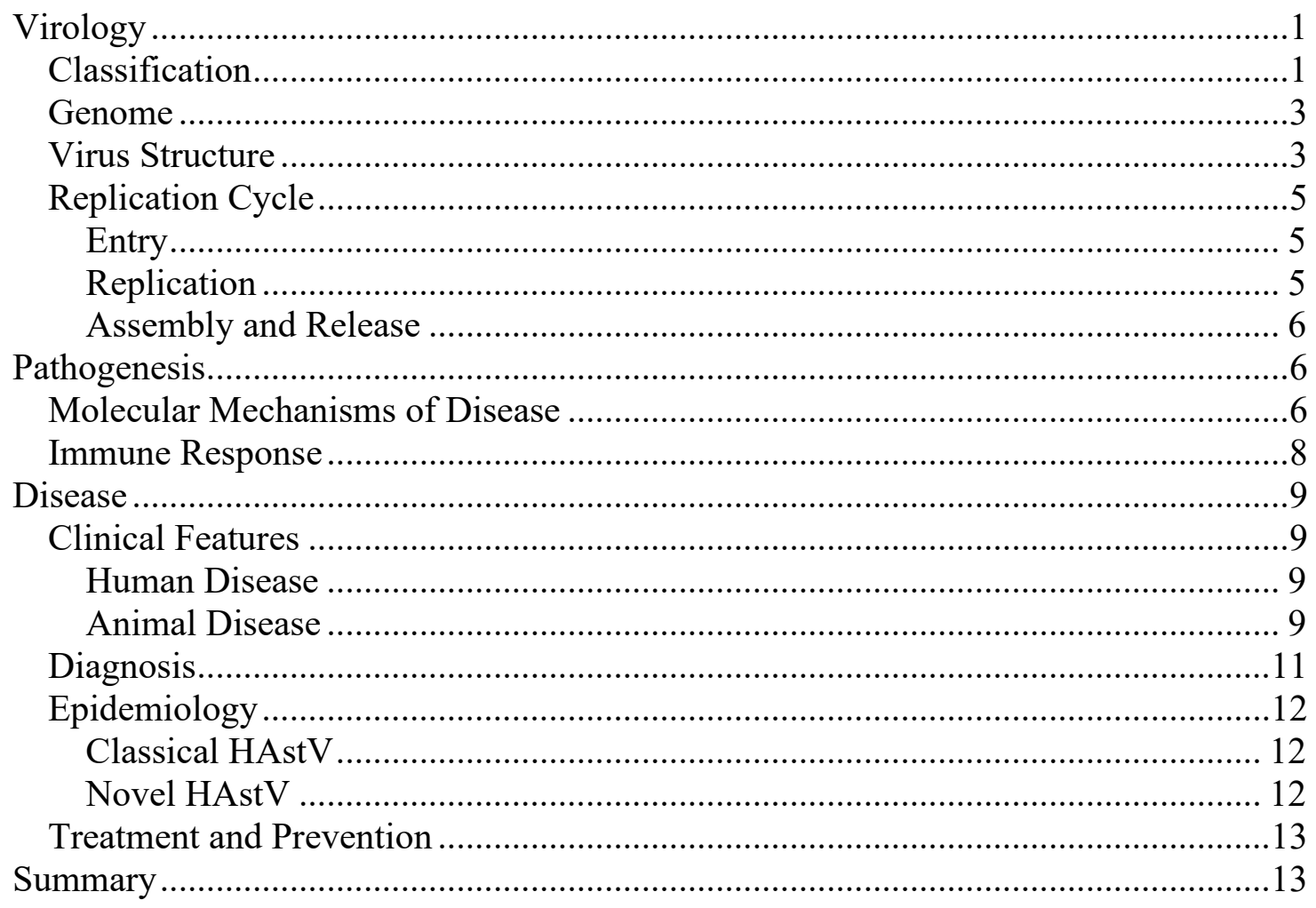

CHAPTER 2. EPITHELIAL-MESENCHYMAL TRANSITION................................15

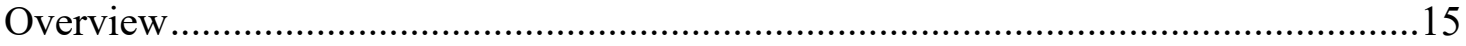

Phenotypic and Genetic Changes ........................................................................15

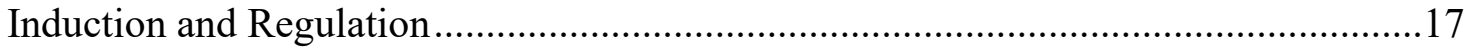

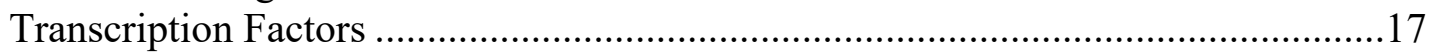

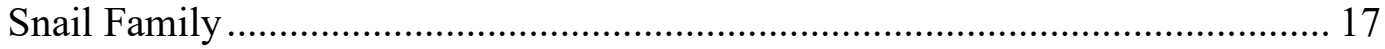

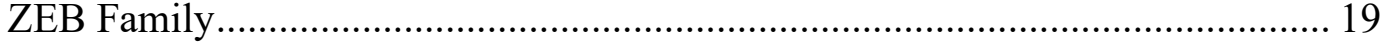

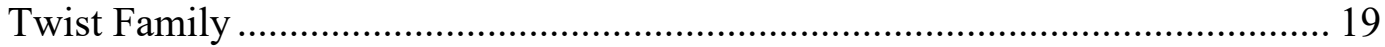

Signaling Pathways ………………………………..................................19

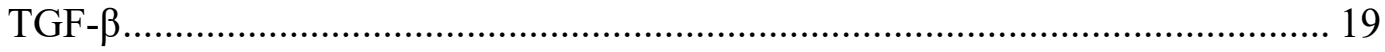

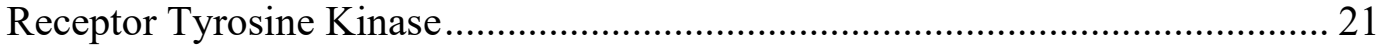

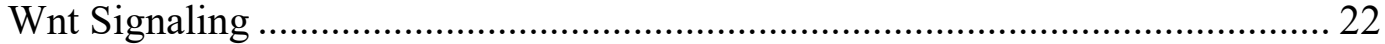

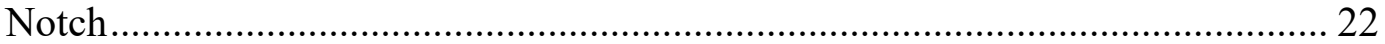

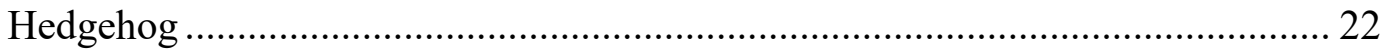

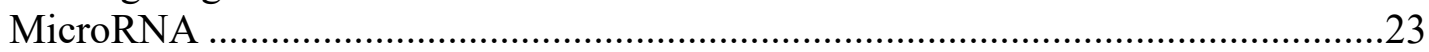

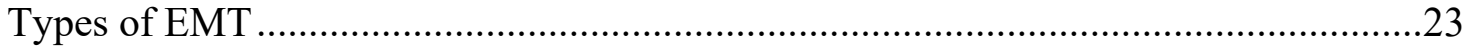

Type 1: Embryogenesis................................................................................23

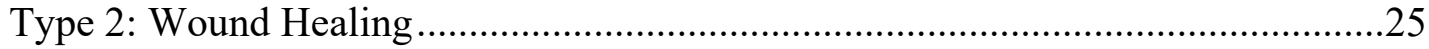

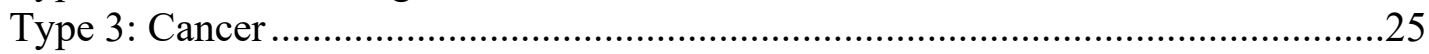

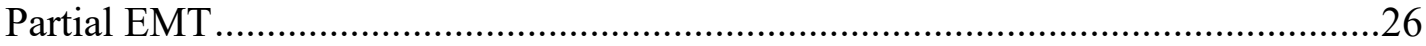




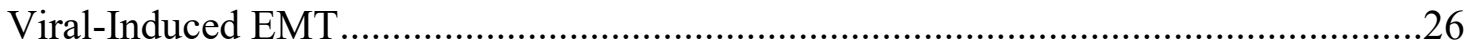

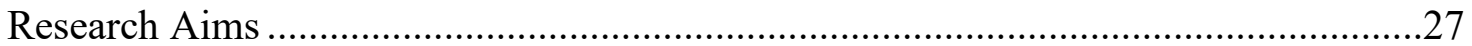

Aim 1: Define the Extent of Astrovirus-Induced EMT ...........................................27

Aim 2: Examine the Mechanism(s) for Astrovirus-Induced EMT ............................27

CHAPTER 3. MATERIALS AND METHODOLOGY .................................................28

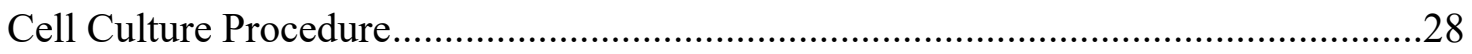

Reagents and Methods Related to HAstV-Induced EMT ..............................................28

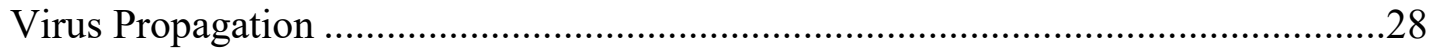

Immunofluorescent Staining and Confocal Imaging .............................................28

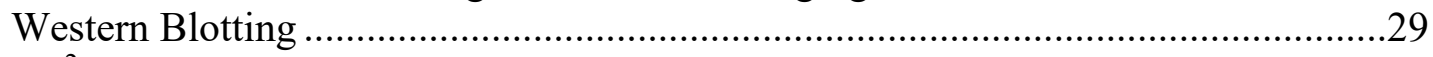

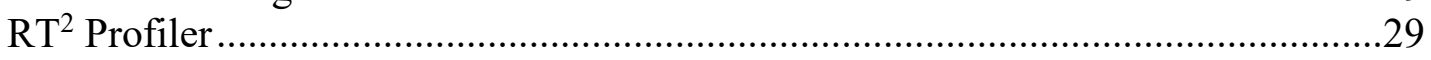

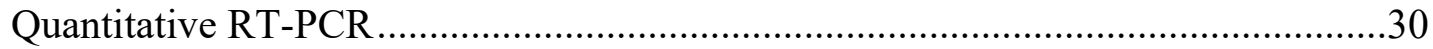

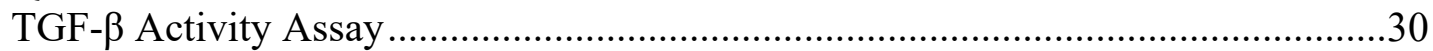

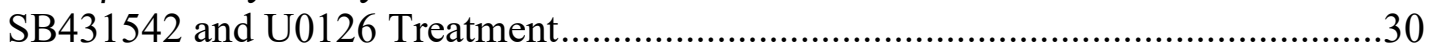

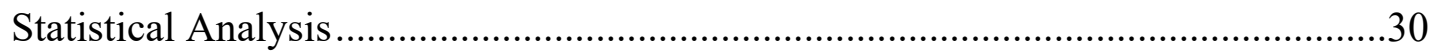

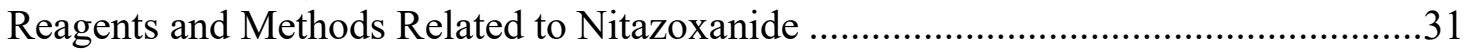

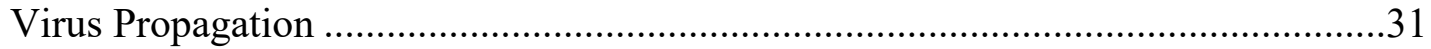

In Vitro HAstV Infection and NTZ Administration..................................................31

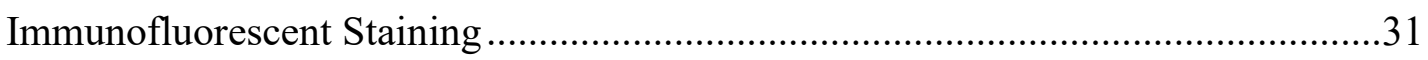

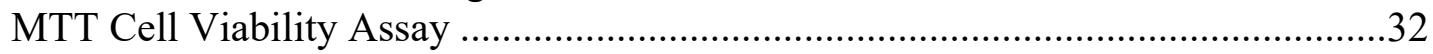

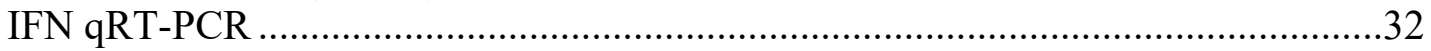

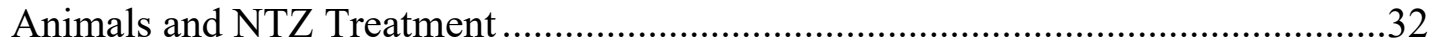

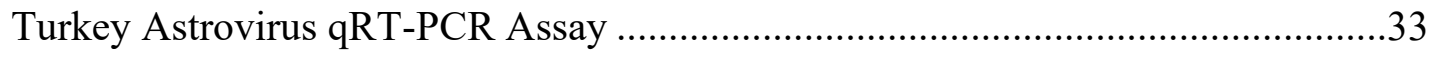

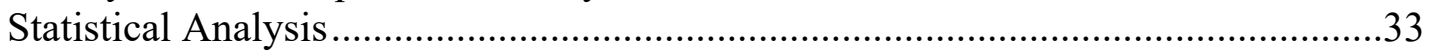

Transepithelial Electrical Resistance (TER) ………..................................................33

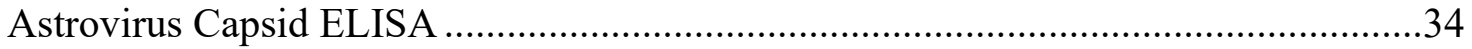

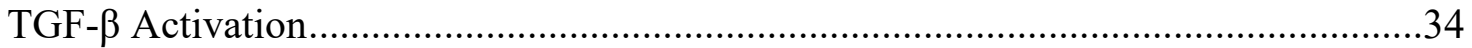

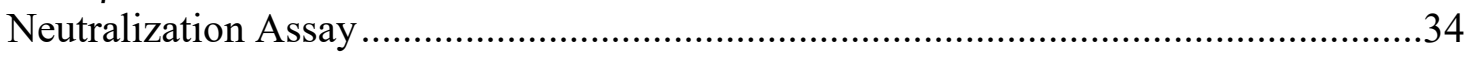

\section{CHAPTER 4. ASTROVIRUS INDUCES REPLICATION-DEPENDENT}

EPITHELIAL-MESENCHYMAL TRANSITION IN CACO-2 INTESTINAL

EPITHELIAL CELLS................................................................................................35

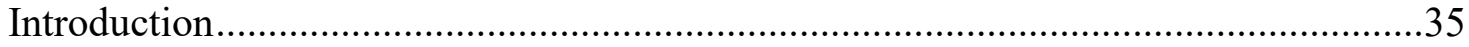

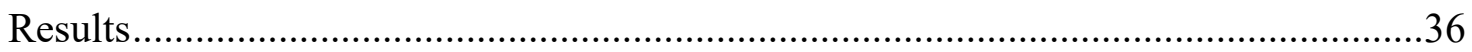

HAstV Infection Leads to Time-Dependent Reorganization and Decrease of Junctional Protein Expression..................................................................................36

HAstV Infection Leads to EMT-Associated Gene Modulation...................................36

HAstV-Induced EMT Disrupts Cellular Polarity ........................................................39

TGF- $\beta$ Activity Is Increased during HAstV Infection .................................................39

Inhibition of TGF- $\beta$ Activity Does Not Prevent HAstV-Induced EMT ......................44

HAstV-Induced EMT Is Replication Dependent .......................................................44

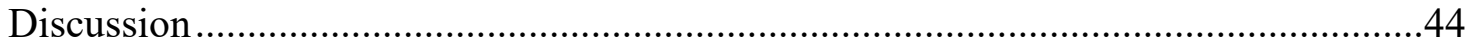

\section{CHAPTER 5. ASTROVIRUS REPLICATION IS INHIBITED BY} NITAZOXANIDE IN VITRO AND IN VIVO.......................................................52 


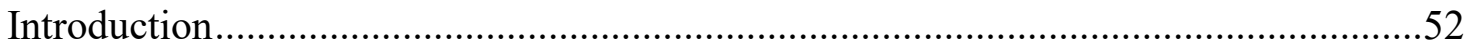

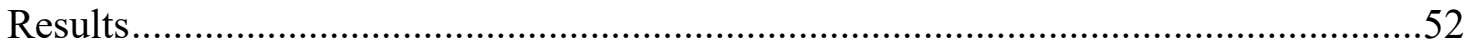

Nitazoxanide (NTZ) Blocks Astrovirus Replication In Vitro ....................................52

NTZ Inhibits dsRNA Production ........................................................................53

NTZ Is Effective Across HAstV Serotypes ..............................................................53

NTZ Reduces Viral Replication and Clinical Disease In Vivo ...................................60

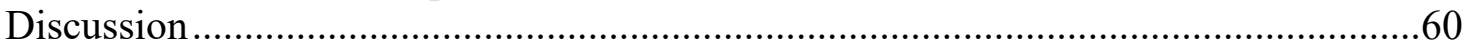

CHAPTER 6. CONCLUSIONS AND FUTURE DIRECTIONS ..................................63

LIST OF REFERENCES ..................................................................................68

APPENDIX A. PRO-INFLAMMATORY CYTOKINES ARE NOT

RESPONSIBLE FOR HASTV-INDUCED EMT ...........................................................107

APPENDIX B. HASTV DIRECTLY ACTIVATES LATENT TGF-B ......................108

APPENDIX C. CLINICAL ISOLATE WITHOUT SEQUENCE HOMOLOGY

TO MIR-487A SHOWS REDUCED EMT CHARACTERISTICS...........................109

APPENDIX D. THE ROLE OF OCCLUDIN IN HASTV INFECTION ...................110

APPENDIX E. HASTV-VA1 ANTIBODIES ARE MORE ABUNDANT THAN

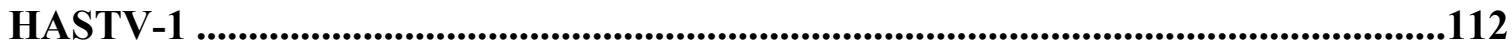

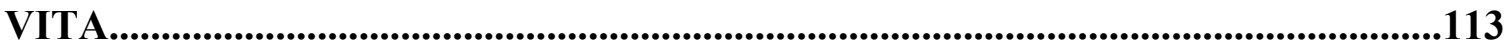




\section{LIST OF TABLES}

Table 1-1. Reports of HAstV Detected in Cases of Meningitis or Encephalitis............10 


\section{LIST OF FIGURES}

Figure 1-1. Phylogenetic Tree of Astroviruses..........................................................2

Figure 1-2. Astrovirus Genome and Capsid Structure...............................................4

Figure 2-1. Organization of Cellular Junction Complex. ..........................................16

Figure 2-2. Epithelial-Mesenchymal Transition (EMT) Characteristics.......................18

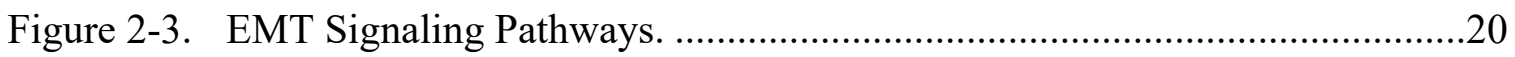

Figure 2-4. MicroRNA Processing and Function...................................................24

Figure 4-1. HAstV-1 Infection Leads to a Time-Dependent Reorganization and Decrease in Junctional Protein Levels. .........................................................37

Figure 4-2. EMT-Associated Genes Modulated by HAstV Infection. ...........................38

Figure 4-3. HAstV-1 Infection Leads to a Decrease in Epithelial Markers while Increasing Mesenchymal Markers..........................................................40

Figure 4-4. HAstV-1 Infection Leads to a Disruption of Cellular Polarity....................42

Figure 4-5. TGF- $\beta$ Activity Increases during the Course of HAstV-1 Infection............43

Figure 4-6. Inhibition of TGF- $\beta$ Signaling with SB431542 Does Not Inhibit HAstV-

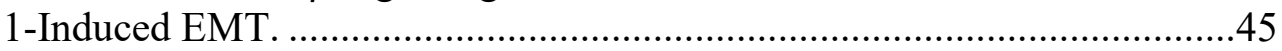

Figure 4-7. TGF- $\beta$-Induced EMT in Caco-2 Cells. .................................................46

Figure 4-8. Replication Is Required for HAstV-1-Induced EMT..............................47

Figure 5-1. Nitazoxanide (NTZ) Inhibits HAstV-1 Replication in Caco-2 Cells...........54

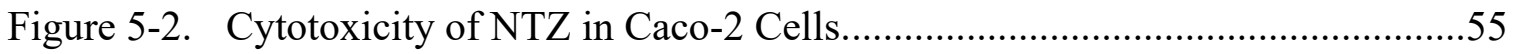

Figure 5-3. Nitazoxanide Inhibits HAstV-1 Replication In Vitro When Added Up to

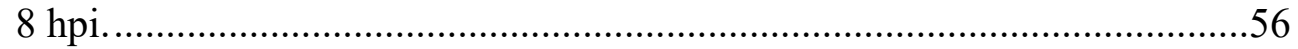

Figure 5-4. NTZ Does Not Induce Significant IFN Levels in Caco-2 Cells. .................58

Figure 5-5. Nitazoxanide Inhibits the Replication of Multiple Serotypes and Clinical Isolates of Human Astrovirus..................................................................59

Figure 5-6. Nitazoxanide Reduces Clinical Symptoms and Viral Titers in Turkey Poults. 
Figure A-1. Pro-Inflammatory Cytokines Are Not Responsible for HAstV-Induced

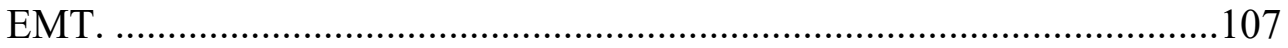

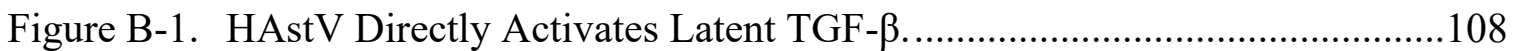

Figure C-1. Clinical Isolate Without Sequence Homology to miR-487a Shows Reduced EMT Characteristics...................................................................109

Figure D-1. Occludin Knockdown Caco-2 Cells............................................................110

Figure D-2. Occludin Knockdown Cells Exhibit Increased Infection.............................111

Figure E-1. HAstV-VA1 Antibodies Are More Abundant than HAstV-1....................112 


\section{LIST OF ABBREVIATIONS}

\begin{tabular}{|c|c|}
\hline AstV & Astrovirus \\
\hline BCA & Bicinchoninic acid \\
\hline $\mathrm{bH} H \mathrm{H}$ & Basic helix-loop-helix \\
\hline BMP & Bone morphogenetic proteins \\
\hline BSA & Bovine serum albumin \\
\hline CAstV & Chicken astrovirus \\
\hline CNS & Central nervous system \\
\hline $\mathrm{CtBP}$ & C-terminal binding protein \\
\hline DAPI & 4',6'-diamidino-2-phenylindole \\
\hline ECM & Extracellular matrix \\
\hline EGF & Epidermal growth factor \\
\hline EMT & Epithelial-mesenchymal transition \\
\hline ERK & Extracellular signal-regulated kinases \\
\hline FBS & Fetal bovine serum \\
\hline FGF & Fibroblast growth factor \\
\hline FFU & Focus forming-units \\
\hline FITC & Fluorescein isothiocyanate \\
\hline HAstV & Human astrovirus \\
\hline $\mathrm{HH}$ & Hedgehog \\
\hline HIF-1 $\alpha$ & Hypoxia-inducible factor $1 \alpha$ \\
\hline $\mathrm{HMO}$ & Human, mink, ovine-like \\
\hline HPI & Hours post-infection \\
\hline
\end{tabular}




$\begin{array}{ll}\text { IFN } & \text { Interferon } \\ \text { IgG } & \text { Immunoglobulin G } \\ \text { iNOS } & \text { Inducible nitric oxide synthase } \\ \text { LEF-1 } & \text { Lymphoid enhancer binding factor-1 } \\ \text { LOX } & \text { Lysyl oxidase } \\ \text { MEM } & \text { Minimal Eagle medium } \\ \text { MET } & \text { Mesenchymal-epithelial transition } \\ \text { MLB } & \text { Melbourne } \\ \text { MMP } & \text { Matrix metalloproteinase } \\ \text { MTT } & \text { 3-(4,5-dimethylthiazol-2-yl)-2,5-diphenyltetrazolium bromide } \\ \text { MuAstV } & \text { Murine astrovirus } \\ \text { NGS } & \text { Normal goat serum } \\ \text { NHE3 } & \text { Sodium-hydrogen antiporter 3 } \\ \text { NICD } & \text { Notch intracellular domain } \\ \text { NO } & \text { Nitric oxide } \\ \text { NSP } & \text { Nonstructural protein } \\ \text { NTZ } & \text { Nitazoxanide } \\ \text { ORF } & \text { Open reading frame } \\ \text { PAGE } & \text { Polyacrylamide gel electrophoresis } \\ \text { PAstV } & \text { Porcine astrovirus } \\ \text { PBS } & \text { Phosphate buffered saline } \\ \text { PES } & \text { Poult enteritis mortality syndrome } \\ \text { RNA dependent RNA polymerase } \\ \text { NR }\end{array}$


RNA Ribonucleic acid

RSS Runting-stunting syndrome

RTK Receptor tyrosine kinase

RT-PCR Reverse transcriptase polymerase chain reaction

SDS Sodium dodecyl sulfate

SGLT1 Sodium/glucose cotransporter 1

sgRNA Subgenomic RNA

SHH Sonic hedgehog

TAstV Turkey astrovirus

TCF T cell factor

TER Transepithelial electrical resistance

TGF- $\beta \quad$ Transforming growth factor $\beta$

UV Ultraviolet

VA Virginia

VPg Viral genome linked protein

VLP Virus like particle

ZEB Zinc finger E-box-binding homeobox

ZO Zonula occludens 


\section{CHAPTER 1. ASTROVIRUS VIROLOGY, PATHOGENESIS, AND DISEASE}

\section{Virology}

Astroviruses are small, non-enveloped, single stranded, positive sense RNA viruses that infect a wide variety of host species. Human astroviruses (HAstV) consistently rank among the leading causes of diarrhea especially in the young, elderly and the immunocompromised. Yet disease can range from asymptomatic to severe central nervous system (CNS) symptoms including encephalitis and meningitis. Despite the dire need for further research, much astrovirus virology and pathogenesis remains understudied. This chapter summarizes the body of literature published on astrovirus while highlighting the areas where further research is needed to better understand this interesting virus.

\section{Classification}

In 1975, astrovirus was first discovered in the stool of infants with gastroenteritis. ${ }^{1}$ It was named for the star-like morphology of the virions' capsid under electron microscopy noted by Madeley and Cosgrove. ${ }^{2,3}$ With the availability of the complete viral genome sequence in 1993, astrovirus was classified into a new family, Astroviridae. ${ }^{4}$ Now, Astroviridae is divided into two genera: Avastrovirus, viruses infecting avian species, and Mamastrovirus, viruses infecting mammalian species ${ }^{5}$ (Figure 1-1). Avastrovirus and Mamastrovirus are further split into two genogroups based on the genetic relatedness within the hypervariable capsid protein.

Following the discovery of HAstV, eight closely related but genetically distinct serotypes were identified. These eight serotypes are known as human astrovirus type 1 through 8 (HAstV-1-8) and are commonly referred to in the field as the classical HAstV. In 2008, using new unbiased full-genome sequencing and pathogen discovery techniques, two additional HAstV clades were identified and named for the locations where they were discovered: Melbourne (HAstV-MLB) and Virginia (HAstV-VA/HMO). ${ }^{6-10}$ HAstV-VA-like viruses were also discovered in Nigeria, Pakistan, and Nepal ${ }^{11}$ and termed HMO because of their genetic similarity to human, mink and ovine astroviruses. To date, the HAstV-MLB clade contains at least three strains, MLB1, ${ }^{8,9,12}$ MLB2, ${ }^{6,10}$ and MLB3,${ }^{6,10}$ and the HAstV-VA/HMO clade contains five strains, VA1, ${ }^{7,11} \mathrm{VA} 2,{ }^{11} \mathrm{VA} 3,{ }^{11}$ VA4, ${ }^{6}$ and VA5. ${ }^{13}$ The HAstV-MLB and HAstV-VA/HMO viruses have been designated as non-canonical or novel human genotypes, due to their genetic relatedness to nonhuman, mammalian astroviruses rather than the classical. 


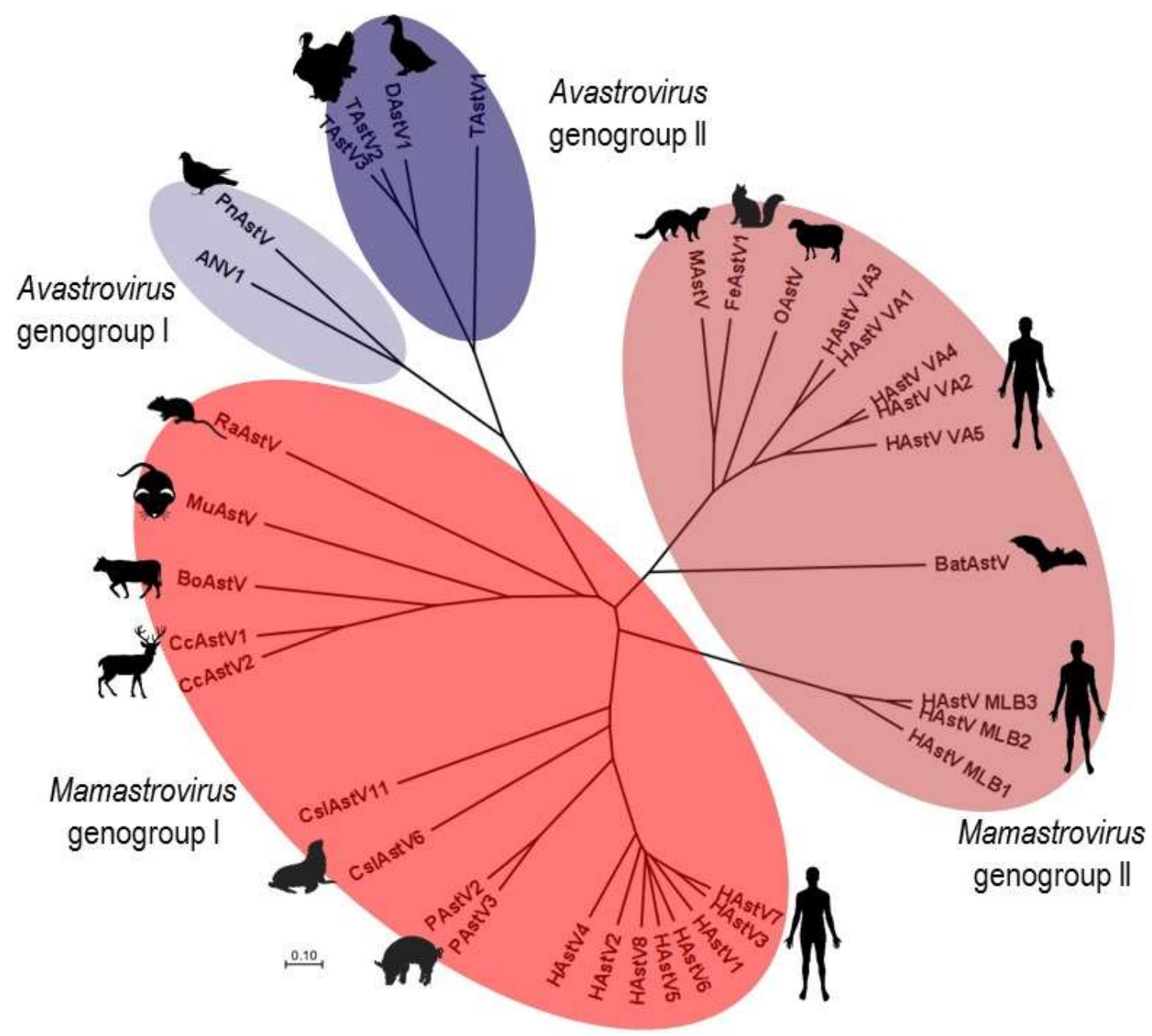

Figure 1-1. Phylogenetic Tree of Astroviruses.

General phylogeny tree of Astroviridae constructed using capsid protein amino acid sequences. Astroviridae is divided into four genogroups based on whether the species a strain infects is mammalian or avian and the genetic relatedness within the hypervariable capsid protein. 


\section{Genome}

Astroviruses belong to group IV of the Baltimore classification of viruses as they have positive sense, single stranded RNA genomes approximately 6.2 to $7.7 \mathrm{~kb}$. The genome has three open reading frames (ORFs) with a 5' viral genome-linked protein (VPg) and a 3' poly(A) tail ${ }^{4,14,15}$ (Figure 1-2A). ORF1a and ORF1b encode the nonstructural proteins. Astrovirus is believed to have as many as five nonstructural proteins, ${ }^{16-22}$ however, only three, a serine protease, a viral genome linked protein (VPg), and an RNA-dependent RNA polymerase (RdRp), ${ }^{22}$ have been fully described. There is a ribosomal frameshift signal between ORF1a and ORF1b which allows for the translation of the RdRp. ${ }^{14,23,24}$ ORF2 encodes the astrovirus capsid protein and is expressed in excess via a highly conserved subgenomic RNA (sgRNA) promoter. This allows for astrovirus capsid protein to be translated at a higher level for assembly of new virions during the viral replication cycle.

\section{Virus Structure}

The star-like appearance of astrovirus noted on about 10 percent of virions by Madeley and Cosgrove is due to spikes studded on the virion surface. ${ }^{3,25}$ The crystal structures for the HAstV-8 spike, ${ }^{26} \mathrm{HAstV}-1$ full capsid, ${ }^{27,28} \mathrm{HAstV}-2$ spike, ${ }^{29}$ and turkey astrovirus type 2 (TAstV-2) spike ${ }^{30}$ have been described using electron and cryo-electron microscopy, X-ray crystallography, and density ultracentrifugation. Infectious astrovirus particles are between 41 and $43 \mathrm{~nm}$ in diameter with $\mathrm{T}=3$ icosahedral symmetry (Figure 1-2B) with significant similarities to the human hepatitis E virus capsid. ${ }^{28,31}$

The capsid protein is translated as an inactive $90 \mathrm{kDa}$ precursor, known as VP90, which is sequentially cleaved before it can be assembled into an infectious viral particle. All capsid protein cleavage products are named according to their molecular weights. Intracellular caspases are the first to cleave VP90, generating VP70. ${ }^{32,33}$ Multiple reports have shown this caspase cleavage is essential for the release of newly formed virions. ${ }^{19,33}$ Once outside the cell, host proteases further cleave VP70 of the immature virions into VP34, VP27, and VP25. ${ }^{22,34}$ VP34 makes up the continuous inner core, measuring about $35 \mathrm{~nm}$ in diameter, while VP27 and VP25 form the spike domains. ${ }^{35}$ While the immature virion has about 90 spikes, the proteolytic cleavage process reduces that to just 30 spikes on a mature, infectious particle. ${ }^{31}$ These spikes are thought to hold multiple putative receptor-binding sites. ${ }^{29,34-36}$

The astrovirus capsid appears to also play a key role in the pathogenesis of the virus. Studies have shown the capsid binds complement $1 \mathrm{q}^{37}$ and mannose-binding lectin $^{38}$ to inhibit both the classical and lectin pathways of complement. This may be why there is not significant immune infiltration or histological changes during astrovirus infection. ${ }^{39,40}$ In addition, the capsid has been shown to act as a novel enterotoxin in vitro ${ }^{41}$ and in vivo, ${ }^{42}$ increasing barrier permeability independent of viral replication. 


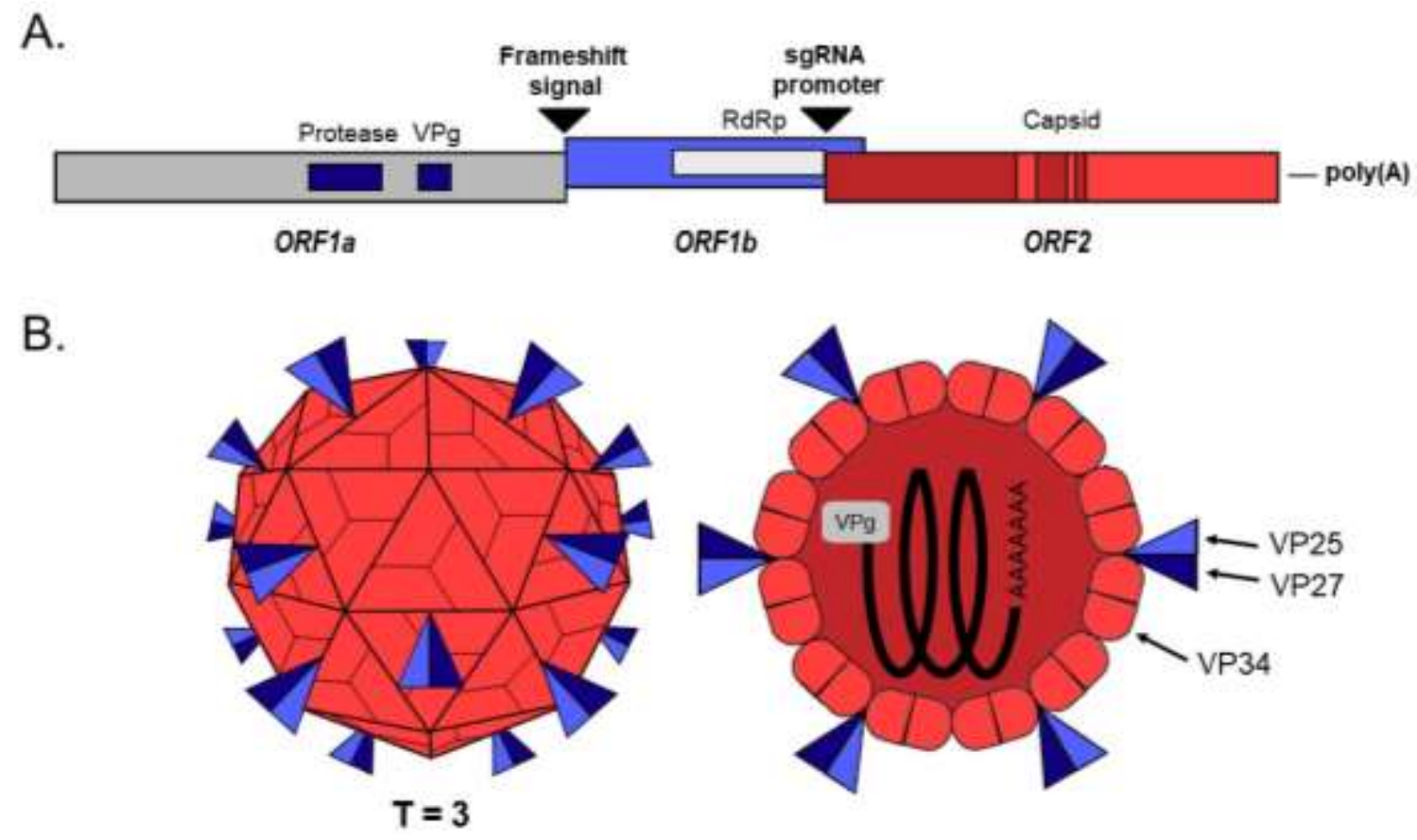

Figure 1-2. Astrovirus Genome and Capsid Structure.

(A) The astrovirus genome is comprised of three open reading frames (ORF) as well as a 5' viral genome linked protein (VPg) and a 3' poly(A) tail. The known encoded proteins are shown in the inset boxes. The lighter portions of ORF2 represent the hypervariable regions. (B) Astrovirus virions have $\mathrm{T}=3$ icosahedral symmetry. The capsid composed by an inner core of VP34 and spike domains composed of VP25 and VP27. 


\section{Replication Cycle}

Many aspects of the astrovirus replication cycle remain undescribed. However, with studies using a few of the classical HAstV genotypes, along with knowledge of other closely related group IV viral families including Togaviridae and Caliciviridae, we know the basic steps of the astrovirus replication cycle.

\section{Entry}

Despite dedicated research, the cellular receptor for astroviruses is still unknown. Studies have shown different cell types do not share the same susceptibilities for HAstV serotypes. For instance, human colorectal adenocarcinoma (Caco-2) cells are the gold standard for HAstV infection and can support the replication of all classical HAstV serotypes; however, baby hamster kidney (BHK-21) cells are only infected by HAstV-2, and human colorectal carcinoma epithelial (HTC-15) cells are only infected by HAstV$1 .^{43}$ This supports the hypothesis that multiple receptors or binding factors may be needed for cell entry. Crystallization of both the avian and human capsid and spike proteins revealed conserved polysaccharide binding motifs ${ }^{30}$ and pretreatment of Caco- 2 cells with chondroitinase, but not heparinase, reduced infectivity. ${ }^{26,29} \mathrm{We}$ also know astrovirus disrupts cellular junction organization, ${ }^{41}$ which may further allow for a binding cascade leading to a previously hidden cellular receptor. Nevertheless, additional research is needed to describe the viral binding step.

Once the virus is bound to a host cell, studies using HEK293 and Caco-2 cells have confirmed entry proceeds via clathrin-mediated endocytosis. ${ }^{44,45}$ Using electron microscopy, astrovirus particles were detected in coated pits and vesicles. ${ }^{44}$ In addition, inhibiting clathrin assembly with chlorpromazine and dansylcadaverine reduced HAstV-8 replication. ${ }^{45}$ After endocytosis, vesicles containing astrovirus particles are trafficked to progressively more acidic endosomes and must reach late endosomes to uncoat the viral genome. Studies blocking the acidification of endosomes with bafilomycin A1 and silencing Rab7 significantly reduced HAstV-8 replication. ${ }^{45}$ Investigation of recombinant viral-like particles (VLPs) identified divalent cations that stabilize the capsid structure. ${ }^{46}$ Therefore, an acidic environment and a low cation would both promote destabilization of the capsid and uncoating of the genome.

\section{Replication}

HAstV genome replication occurs in intracellular membrane bound vesicles. ${ }^{47}$ Given that HAstV nonstructural polyprotein contains an endoplasmic reticulum (ER) signal sequence and both the nonstructural proteins and genome colocalize with the ER, ${ }^{48}$ it is assumed these vesicles are derived from ER membrane. Since the viral genome is positive-sense RNA, following release, it can be directly translated into protein. The first proteins made after viral uncoating are the nonstructural proteins, nspla and nsplab, encoded by ORF1a and ORF1b. The nsplab polyprotein is processed by the viral protease, and possibly by yet unidentified host proteases, to form the nsp $1 \mathrm{~b}$ protein, the viral RdRp. ${ }^{14}$ The nspla polyprotein is cleaved into several smaller proteins, some of 
whom have an undetermined function. ${ }^{16,18,20,22,49}$ One of the nspla proteins, nsp $1 \mathrm{a} / 4$, a putative VPg, has been shown to colocalize with viral genome and is thought to facilitate viral replication. ${ }^{19,48,50}$ Although direct interaction between nsp1a/4 and the genome has not been established, the production of infectious virus was significantly reduced when the viral RNA was pretreated with proteinase $\mathrm{K}$ before transfection into permissive cells, ${ }^{51}$ indicating some protein interaction is required for efficient replication. The production of the capsid protein from ORF2 involves translation of both genomic and sgRNA. Around 12 hours post-infection there is an excess of sgRNA produced, which coincides with higher levels of capsid protein translation. ${ }^{52}$ This is characteristic utilized by other positive-sense single-stranded RNA viruses meant to evade translational repression by the host. ${ }^{53}$

We know multiple host proteins are necessary for astrovirus replication, however, their exact role has yet to be fully elucidated. One study, using density gradient centrifugation, free-flow zonal electrophoresis, and tandem mass spectrometry, identified host proteins that associated with the same membrane fraction as viral proteins and RNA. ${ }^{54}$ Silencing host proteins involved in fatty acid and cholesterol synthesis, phosphatidylinositol and inositol metabolism, and RNA helicase activity, all decreased the production of viral RNA, protein and infectious progeny of HAstV $-8 .{ }^{54}$ Our lab has demonstrated ERK1/2 is phosphorylated within 15 min of viral binding and this phosphorylation event is required for efficient viral protein production and genome replication. ${ }^{55}$ It has recently been determined the ubiquitin proteasome system is also needed for replication of the viral genome and $\operatorname{sgRNA} .^{56}$

\section{Assembly and Release}

In the same membraned sites where replication takes place, viral capsids can selfassemble into full virions or virus like particles (VLP) without the presence of a viral genome. ${ }^{46}$ Virions are processed and release occurs via an unknown, non-lytic mechanism without causing significant cell death. ${ }^{33,57}$ Researchers have hypothesized that astrovirus release may occur through a similar mechanism used by rotavirus or poliovirus, or through a form of cell membrane destabilization. ${ }^{57}$

\section{Pathogenesis}

\section{Molecular Mechanisms of Disease}

Many astrovirus pathogenesis studies have been concentrated on elucidating the mechanism by which the virus induces diarrhea. It is common for gastrointestinal pathogens to breach the intestinal epithelium by inducing inflammation and cell damage or death, and in doing so cause diarrhea. However, this is not the case with HAstV. Astrovirus-induced diarrhea occurs without triggering cell death or inflammation, ${ }^{39,42}$ although one report demonstrated that $\mathrm{HAstV}-8$ induced apoptosis in Caco- 2 cells. ${ }^{33}$ Rather, HAstV is thought to cause diarrhea by increasing barrier permeability via the 
disruption of intestinal cellular junctions. ${ }^{41,42,58,59}$ Cellular junctions are highly regulated, multiprotein complexes that join neighboring cells, maintain cellular polarity, and regulate the passage of nutrients, solutes and microorganisms across the epithelium. ${ }^{60,61}$ These junctions are comprised of the tight junction complex, which includes transmembrane proteins, such as occludin and claudin, and cytosolic adapters such as zonula occluden 1 (ZO-1), and the adherens junctional complex including cadherins and $\beta$-catenin. ${ }^{61}$

Astrovirus infection alters intestinal barrier permeability via redistribution of junctional proteins. In vitro $\mathrm{HAstV}$ infection of Caco- 2 cells induces a drop in transepithelial electrical resistance (TER) and allows for greater flux of fluorescein isothiocyanate (FITC)-labeled dextran across the cell monolayer. ${ }^{41}$ The decrease in TER and increase in flux is first observed between 16 and 20 hours post-infection (hpi), with the greatest permeability seen between 36 and $48 \mathrm{hpi}$, and could be attributed to a reduction in occludin from the junctional complex and a reduction in F-actin fibers. ${ }^{41}$ This phenomenon has also been described in vivo. One study of murine astrovirus (MuAstV) reports an increase intestinal permeability, as measured by orally administered FITC-labeled dextran crossing the intestinal barrier and reaching the bloodstream. ${ }^{58}$ Investigations using the turkey poult model present similar results. Infected poults show increased lumenal-to-serosal flux and disruption of F-actin fibers, ${ }^{59}$ as well as the disruption of the localization of the sodium transport proteins NHE3 and SGLT-1. ${ }^{42,59}$ These findings support the hypothesis that astrovirus may disrupt ionic transport causing sodium malabsorption and osmotic diarrhea.

Intriguingly, the disruption of barrier integrity associated with astrovirus infection occurs without active viral replication. Moser et al. found both UV-inactivated HAstV and recombinant capsid protein increase barrier permeability in Caco- 2 cells. ${ }^{41}$ In addition, in vivo administration of the recombinant TAstV-2 capsid protein to turkey poults induces acute diarrhea and relocalization of SGLT-1, comparable to infected animals. $^{42}$

More recently studies have focused on defining the cellular tropism for HAstV in hopes of developing better models to study infection. Initial studies show astrovirus infects mature intestinal enterocytes at the tips of the villi, macrophages of the lamina propria, and possibly $\mathrm{M}$ cells. ${ }^{62-64}$ Novel HAstV-VA genotypes are thought to be neurotropic, as primary astrocytes and SK-N-SH cells are permissive to HAstV-VA1 and HAstV-4 infection. ${ }^{65}$ However, viruses from the HAstV-VA and HAstV-MLB clades can also be propagated in Caco-2, Vero, A549, and Huh7 cell lines as well. ${ }^{66-68}$ Studies in human intestinal enteroids reveal that along with enterocytes, HAstV-VA1 infects intestinal progenitor cells. ${ }^{69}$ Interestingly, MuAstV is shown to infect actively secreting goblet cells. ${ }^{70}$ When mucus production is disrupted, there is a subsequent reduction in viral infection and shedding. ${ }^{70}$ Further, the increase in mucus production seen during MuAstV infection promotes alterations in the gut microbiome that can lead to resistance of some enteric pathogens. ${ }^{70}$ 


\section{Immune Response}

Little is known about the immune response to astrovirus. Multiple studies have shown infection does not produce overt inflammation unlike many other enteric pathogens. ${ }^{39,40}$ Given the acute nature of most astrovirus infections, it is assumed that the innate immune system is essential for controlling the virus. HAstV replication is sensitive to the type I interferon (IFN) response, an important part of innate immunity. Two studies using classical HAstV serotypes showed that IFN- $\beta$ levels increase after infection and pretreatment with exogenous type I IFN can reduce replication in Caco-2 cells and protect epithelial barrier integrity. ${ }^{58,71}$ Unlike the increase in barrier permeability, productive replication is required to induce IFN- $\beta$ in vitro as neither $\mathrm{UV}$-inactivated virus nor recombinant capsid protein increased IFN- $\beta$ mRNA levels. ${ }^{58,71}$ Recently, studies using HAstV-VA1 in human intestinal enteroids demonstrated a strong type I and III IFN response is triggered by novel HAstV as well. ${ }^{69}$ In vivo studies showed that IFNAR knockout mice, which lack the type I IFN receptor, cannot clear MuAstV infection unlike wild-type mice. ${ }^{58}$

Along with inhibiting complement factors as mentioned previously, astrovirus may modulate the immune response by activating transforming growth factor- $\beta$ (TGF- $\beta$ ), inducing nitric oxide (NO) production, and impairing macrophage function. In TAstV-2 infected turkey poults active TGF- $\beta$ levels are elevated compared to uninfected controls. ${ }^{39}$ TGF- $\beta$ is a known immunosuppressive cytokine and may contribute to the lack of inflammation seen during astrovirus infection. While astrovirus has been shown to infect macrophages, this infection does not lead to productive replication. ${ }^{72}$ It does however decrease macrophage function and viability. ${ }^{73}$ Also, TAstV-2 infected macrophages along with infected epithelial produce inducible NO synthase (iNOS) triggering the production of NO. ${ }^{72,74}$ Interestingly, that NO production inhibits astrovirus replication in turkey poults and treatment with NO synthase inhibitors in vitro and in vivo increased TAstV-2 replication, ${ }^{72}$ highlighting the role of NO in suppressing astrovirus infection.

The immediate response of the innate immune system allows for the initial control of astrovirus and facilitates specific humoral and cell-mediated adaptive responses. More than 70 percent of healthy adults have detectable HAstV-specific antibodies. ${ }^{75}$ Nearly 90 percent of children have antibodies to HAstV, so the decrease in the percent of seropositive adults suggests humoral immunity may wane over time. Volunteer studies have shown the association between HAstV-specific humoral immunity and disease outcome. Subjects with HAstV antibodies have less severe symptoms compared to those without. $^{76,77}$ Analysis of neutralizing antibodies from several genotype revealed a quaternary epitope on each side of the dimeric spike domain necessary for neutralization. ${ }^{78}$ These studies will help inform decisions for future vaccine development if a vaccine is ever needed.

In respect to cell-mediated adaptive response, $\mathrm{T}$ cells are also believed to be necessary for viral clearance. In a challenge of biopsied tissue from the small intestine of healthy adults, CD4+ T cells specific for HAstV were found to reside in the small 
intestine. ${ }^{79}$ Recent MuAstV research has shown Rag1 knockout mice, which lack B and T cells, experience prolonged viral shedding in their feces compared to their wild-type counterparts. ${ }^{80}$ Rag1 knockout mice also exhibit higher viral titers in the intestines, mesenteric lymph nodes, spleen, liver, and kidneys, demonstrating the importance of B and $\mathrm{T}$ cells in preventing systemic spread of astrovirus.

\section{Disease}

\section{Clinical Features}

Volunteer studies confirmed that astrovirus is transmitted via the fecal-oral route. ${ }^{76,77}$ We now know the virus can be transmitted through contaminated food, water, and fomites as well as human-to-human, and is a common nosocomial infection.

\section{Human Disease}

HAstV infection typically manifests as watery diarrhea that last 2 to 4 days, although fever, headaches, abdominal pain and anorexia can also occur. ${ }^{81-83}$ However, healthy individuals with HAstV infection may not present with any symptoms. ${ }^{84}$ It is thought that a large proportion of HAstV infections are asymptomatic, ${ }^{85}$ which may be attributed to immunity from previous exposure. ${ }^{76,77,86}$

While in healthy individuals HAstV infections are quite mild, increasing reports indicate in the immunocompromised population, HAstV infections are associated with severe symptoms and extra-gastrointestinal dissemination. ${ }^{87}$ The first report suggesting HAstV to be the causative agent of encephalitis and meningitis was in the 2010 case of a 15 -year old boy with X-linked agammaglobulinemia. ${ }^{88}$ Virus specific primers were unable to detect the responsible pathogen; however, next-generation sequencing (NGS) identified HAstV-VA1 in the biopsy samples from the patient. Subsequently, nine other cases have reported encephalitis and meningitis in association with HAstV infection ${ }^{89-96}$ (Table 1-1). These cases have primarily been in immunocompromised patients, but two cases were in immunocompetent individuals, and in eight of the ten cases, a novel HAstV genotype was identified as the causative agent. These reports demonstrate that HAstV genotypes can cause severe neurologic symptoms rather than just mild diarrhea, highlighting the need for extensive research into astrovirus pathogenesis. Especially now that epidemiological studies show infections caused by novel HAstV genotypes are not as rare as once thought. ${ }^{97}$

\section{Animal Disease}

Similar to infection in humans, diarrhea is the main symptom of astrovirus infection in animal species including calves and cattle, ${ }^{98-100}$ lambs,,${ }^{62,63}$ turkeys, ${ }^{101-105}$ chickens, ${ }^{106,107}$ piglets, ${ }^{108}$ and dogs,${ }^{109}$ among many others. ${ }^{110}$ Likewise, asymptomatic 
Table 1-1. Reports of HAstV Detected in Cases of Meningitis or Encephalitis.

\begin{tabular}{|c|c|c|c|c|c|c|}
\hline Age/Sex & $\begin{array}{l}\text { Underlying } \\
\text { Conditions }\end{array}$ & Presentation & $\begin{array}{r}\text { Site(s) of } \\
\text { Detection }\end{array}$ & Genotype & $\begin{array}{c}\text { Hospitalization } \\
\text { Duration \& } \\
\text { Outcome }\end{array}$ & Reference(s) \\
\hline $15 \mathrm{y} / \mathrm{M}$ & $\begin{array}{l}\text { X-linked } \\
\text { agammaglobulinemia }\end{array}$ & Encephalitis & Brain & $\begin{array}{l}\text { HAstV-VA } \\
\text { /HMO clade }\end{array}$ & $71 \mathrm{~d}$, Death & $\begin{array}{l}\text { Quan et al. } \\
2010^{88}\end{array}$ \\
\hline $3 \mathrm{mo} / \mathrm{M}$ & $\begin{array}{l}\text { SCID, gamma C deficient, } \\
\text { post-allogeneic HSCT }\end{array}$ & $\begin{array}{l}\text { Encephalitis, } \\
\text { MOF }\end{array}$ & $\begin{array}{l}\text { Brain, heart, lung, } \\
\text { spleen, bone marrow, } \\
\text { kidney, small intestine, } \\
\text { stool, plasma }\end{array}$ & HAstV-4 & $17 \mathrm{~d}$, Death & $\begin{array}{l}\text { Wunderli et al. } \\
2011^{89}\end{array}$ \\
\hline $1.5 \mathrm{y} / \mathrm{M}$ & Post-allogeneic HSCT & Encephalitis & Brain & $\begin{array}{l}\text { HAstV-VA1 } \\
\text { /HMO-C }\end{array}$ & 9 mo, Death & $\begin{array}{l}\text { Brown et al. } \\
2015^{92}\end{array}$ \\
\hline $42 \mathrm{y} / \mathrm{M}$ & $\begin{array}{l}\text { CLL, post-allogeneic } \\
\text { HSCT }\end{array}$ & Encephalitis & Brain, CSF & $\begin{array}{l}\text { HAstV-VA1 } \\
\text { /HMO-C }\end{array}$ & $7.5 \mathrm{mo}$, Death & $\begin{array}{l}\text { Naccache et al. } \\
2015^{93}\end{array}$ \\
\hline $14 \mathrm{y} / \mathrm{M}$ & $\begin{array}{l}\text { X-linked } \\
\text { agammaglobulinemia }\end{array}$ & Encephalitis & Brain & $\begin{array}{l}\text { HAstV-VA1 } \\
\text { /HMO-C }\end{array}$ & $\begin{array}{l}>9 \text { mo, } \\
\text { Recovered }\end{array}$ & $\begin{array}{l}\text { Fremond et al. } \\
2015^{93}\end{array}$ \\
\hline $4 y / M$ & $\begin{array}{l}\text { Congenital aplastic } \\
\text { anemia, post-allogeneic } \\
\text { HSCT }\end{array}$ & Encephalopathy & $\begin{array}{l}\text { CSF, stool, serum, } \\
\text { urine, throat }\end{array}$ & HAstV-MLB1 & 19 d, Recovered & $\begin{array}{l}\text { Sato et al. } \\
2016^{91}\end{array}$ \\
\hline $21 \mathrm{y} / \mathrm{F}$ & None & Meningitis & $\begin{array}{l}\text { CSF, anus, urine, } \\
\text { plasma }\end{array}$ & HAstV-MLB2 & 10 d, Recovered & $\begin{array}{l}\text { Cordey et al. } \\
2016^{90}\end{array}$ \\
\hline $37 \mathrm{y} / \mathrm{M}$ & $\begin{array}{l}\text { AML, post-allogeneic } \\
\text { HSCT }\end{array}$ & Meningitis & CSF, plasma, stool & HAstV-MLB2 & $15 \mathrm{mo}$, Death & $\begin{array}{l}\text { Cordey et al. } \\
2016^{90}\end{array}$ \\
\hline $8 \mathrm{mo} / \mathrm{F}$ & $\begin{array}{l}\text { AML, post-allogeneic } \\
\text { HSCT }\end{array}$ & Encephalitis & Brain & $\begin{array}{l}\text { HAstV-VA1 } \\
\text { /HMO-C }\end{array}$ & 196 d, Death & $\begin{array}{l}\text { Lum et al. } \\
2016^{95}\end{array}$ \\
\hline $16 \mathrm{mo} / \mathrm{F}$ & $\begin{array}{l}\text { Immunocompetent, } \\
\text { generalized seizures }\end{array}$ & Encephalitis & CSF, stool & HAstV-1 & 9 d, Recovered & $\begin{array}{l}\text { Koukou et al. } \\
2019^{96}\end{array}$ \\
\hline
\end{tabular}

Abbreviations: AML, acute myeloid leukemia; CLL, chronic lymphocytic leukemia; CSF, cerebrospinal fluid; d, day; F, female; HSCT, hematopoietic stem cell transplant; M, male; mo, month; MOF, multiple organ failure; ND, not determined; SCID, severe combined immunodeficiency; y, year. 
infections in animals have also been reported. ${ }^{98,108,111,112}$ However, other symptoms not associated with the gastrointestinal tract have been described in certain animal species. Chicken astrovirus (CAstV) infection in broiler chicks is associated with the hatchery diseases runting-stunting syndrome (RSS) and "white chicks" condition. ${ }^{113-116}$ Both of these ailments present with small hatch size (runting), decreased weight gain (stunting), increased weakness and mortality, and can be characterized by pale plumage ("white chick"). ${ }^{117}$ Similarly, TAstV infections in turkeys is associated with poult enteritis mortality syndrome (PEMS). ${ }^{118,119}$ PEMS affects young turkeys and is characterized by diarrhea, dehydration, severely stunted growth, and high mortality. ${ }^{120}$ Along with these, encephalitis has been associated with astrovirus infection in mink, cows, and sheep. ${ }^{121-125}$ More recently, porcine astrovirus type 3 (PAstV-3) was detected in tissues from CNS of pigs suffering a neurologic disorder that had symptoms ranging from hind limb weakness to quadriplegia and occasional convulsions. ${ }^{126}$

Until recently, paradigm suggested that astrovirus infections were speciesspecific. However, several avian astroviruses share genetic characteristics indicating that transmission events between species may occur often. ${ }^{127}$ Additionally, there is evidence of cross-species infection or transmission between humans and animals. One study found individuals who work in close contact with poultry are three times more likely to have antibodies to TAstV-2, than individuals without contact with poultry. ${ }^{128}$ Another study has shown evidence of both mammalian and avian astrovirus genotypes in non-human primate fecal samples, as well as HAstV-specific antibodies in plasma samples. ${ }^{129}$ These studies suggest astrovirus may not be species specific as once thought; however, it remains to be seen if infection with cross-species strains would cause symptoms of disease.

\section{Diagnosis}

Originally, HAstV was identified by electron microscopy in positive stool samples. ${ }^{1,3,130}$ In the 1990 's, the first RT-PCR and enzyme-linked immunosorbent assays (ELISA) were developed for detection and typing of $\mathrm{HAstV},{ }^{131-133}$ making diagnosis much less laborious and time consuming. Now, several multiplexed real-time RT-PCR methods exist for quick screening of stool samples for $\mathrm{HAstV}$ and additional enteric viruses, most commonly adenovirus, norovirus, rotavirus and sapovirus. ${ }^{134}$ At the present time, only one multiplexed real-time RT-PCR assay to accurately detects HAstV-1-8, HAstV-MLB1, and HAstV-VA2. ${ }^{135}$ Unfortunately, due to limited availability of genome sequences through public databases, development of a real-time RT-PCR methods to detect all 16 of the known HAstV serotypes has been slow. This is a critical public health need for researchers in order to improve surveillance efforts and more accurately assess prevalence. 


\section{Epidemiology}

\section{Classical HAstV}

Classical HAstV infections are overwhelmingly an affliction of the pediatric population. ${ }^{136-138}$ The typical age range of infection is newborn to 5 years, ${ }^{139-142}$ with up to 90 percent of children ages 5 years and older having detectable HAstV-specific antibodies. ${ }^{83,143,144}$ However, the elderly and immunocompromised are also at risk of infection. ${ }^{97,145-148}$ While less common, cases of HAstV infection in healthy adults have been reported. ${ }^{149-151}$ Although there happens to be higher incidence of infection in winter, possibly due to enhanced stability in colder temperatures, HAstV circulates year-round with infections reported in summer months. ${ }^{152}$

Classical HAstV infection is thought to be associated with 2 to 9 percent of all acute, non-bacterial diarrhea in children. ${ }^{110}$ While incidence of infection decreases with age, HAstV-1 and HAstV-5 were associated with diarrheal outbreaks in elderly care facilities. ${ }^{153,154}$ As of 2013, the mean incidence of HAstV infection was 11 percent globally, with 7 percent incidence in rural areas and 23 percent in urban areas. ${ }^{155}$ Incidence tends to be higher in non-developed countries, likely due to poor sanitation and water treatment methods. Statistics on HAstV infections are significantly affected by underreporting and asymptomatic cases. Fortunately, the increase in sensitivity of realtime RT-PCR has allowed for more asymptomatic HAstV infections to be detected. ${ }^{156-158}$ Using more sensitive screening, the rate of HAstV positivity in asymptomatic children is thought to be roughly 4 percent. ${ }^{152}$ While the incidence of each of the classical serotypes differs depending on geographic location, HAstV-1 is the predominant serotype circulating around the world. ${ }^{83,84,142,159-163}$ Over the past four decades, the incidence of HAstV infection has been decreasing from 22 percent in the 1980 s to about 5 percent in the 2000s. ${ }^{155}$ One hypothesis for this decrease is that novel HAstV genotypes could be outcompeting the classical genotypes, since reports have shown their prevalence is on the rise.

\section{Novel HAstV}

The link between the MLB and VA clades of HAstV and clinical disease is still somewhat unclear, making accurate reporting even more difficult compared to classical genotypes. Reports have exhibited this in the drastic differences in prevalence from one geographic location to another. ${ }^{87}$ The most recent review of novel HAstV prevalence, reports an overall positivity rate 1.5 percent lower than the prevalence of classic HAstV genotypes. ${ }^{87}$ In a study of immunocompromised pediatric oncology patients, 50 percent of samples were positive for HAstV-1; HAstV-VA2 and HAstV-MLB1 were detected in 21 percent and 13 percent of samples, respectively. ${ }^{97}$ However, a study conducted in Japan in 2016, found a positivity rate for HAstV-MLB of 10.6 percent, more than double that of classic HAstV at 5.1 percent. ${ }^{164}$ Although most reports generally describe a low prevalence of novel HAstV, their seroprevalence is surprisingly high. Studies in the US have reported the seropositivity for HAstV-MLB1 to be 86 percent $^{165}$ and HAstV-VA1 to be 65 percent, ${ }^{166}$ indicating a large portion of the population have been infected with 
these viruses in their lifetime. The huge discrepancy between reported prevalence and seroprevalence again suggests that many RT-PCR screening methods are unable to detect all members of the HAstV-VA and HAstV-MLB clades resulting in skewed statistics.

\section{Treatment and Prevention}

To date, there is no vaccine or widely accepted treatments for HAstV infection. This is partially due to the relatively mild symptoms of HAstV infection, which typically resolve without medical intervention. If treatment is necessary, it is generally only to alleviate symptoms. HAstV-induced diarrhea can cause dehydration and therefore may require oral or intravenous fluid administration. Administration of intravenous IgG (IVIG) has been proposed in cases of severe disease in immunocompromised patients, ${ }^{167}$ but the efficacy of such a treatment needs further investigation.

As there are no vaccines for astrovirus, the most effective method of prevention is decontamination of fomites and water. Many healthcare facilities regularly use 70 to 90 percent ethanol to disinfect surfaces; however, this treatment is ineffective at completely eliminating astrovirus. ${ }^{168}$ Treatment with 90 percent methanol on the other hand, reduces astrovirus levels to below the limit of detection. ${ }^{118,168}$ Astrovirus is an incredibly robust virus that is resistant to many detergents, lipid and chlorine solvents and even to $\mathrm{pH}$ as low as $3 .{ }^{118}$ Temperature modulation also has very little effect on astrovirus. It can remain infectious at low temperatures up to ten years, ${ }^{169}$ and heating the virus at $60^{\circ} \mathrm{C}$ for 10 minutes did not affect infectivity. ${ }^{118}$ However, one study has shown that overnight treatment with 0.3 percent formaldehyde and a 30 minute treatment with 1.5 percent Virkon S, a peroxymonosulfate, can fully inactivate the virus. ${ }^{118}$

Astrovirus has been detected in environmental water samples ${ }^{170,171}$ and shown to persist in drinking water, ${ }^{172}$ showing that contaminated water poses a significant threat to spread astrovirus infection. Typical treatment for drinking water involves disinfection with chlorine or chloramine. Astrovirus infectivity has been shown to be significantly decreased by $1 \mathrm{mg} / \mathrm{L}$ free chlorine for 2 hours. ${ }^{172}$ However, in areas with poor water sanitation, astrovirus infection will remain a problem. ${ }^{173,174}$

\section{Summary}

In conclusion, significant progress has been made in the past two decades although there is still much to be learned about astrovirus. The high seroprevalence of HAstV-MLB and HAstV-VA genotypes show that these astrovirus infections are more common than was once thought. Along with the discovery of these novel HAstV genotypes, researchers have developed a greater appreciation for the virus to spread systemically and cause encephalitis especially in immunocompromised hosts. In addition, the need for antivirals to treat these severe cases has been recognized. 
It has also become clear that astrovirus causes disease in an unconventional way. Rather than causing inflammation of the gastrointestinal tract or inducing apoptosis, astrovirus disrupts tight junctions, disrupting barrier integrity, and possibly causing diarrhea. We hypothesize astrovirus induced barrier permeability and disruption of tight junctions is just the beginning of a more complex process called epithelial-mesenchymal transition (EMT). My work will determine if astrovirus is prompting EMT and characterize the mechanism of this process. 


\section{CHAPTER 2. EPITHELIAL-MESENCHYMAL TRANSITION}

\section{Overview}

Epithelial and mesenchymal cells serve different roles in the body. As such, these cells have vastly different phenotypes and gene signatures. Epithelial cells share specialized contacts with neighboring cells to form regulated barriers. These contacts are comprised of tight junctions, adherens junctions, desmosomes, and gap junctions (Figure 2-1). Epithelial cells are also characterized by apical-basolateral polarization, which is defined by the localized distribution of adhesion molecules especially cadherins and certain integrins, the organization of cell-cell junctions as a continuous adhesion belt, the polarized organization of the actin cytoskeleton, and the connection of extracellular matrix at the basal surface ${ }^{175}$. While epithelial cells can become motile and release contacts from their neighboring cell, under normal conditions these cells usually do not detach from the epithelial layer. Conversely, mesenchymal cells are highly motile and lack organization into layers and apical-basolateral polarization. They have spindleshaped, fibroblast-like morphology, only contact neighboring cells focally, and rarely contact the basal lamina.

Epithelial and mesenchymal cells do share inherent plasticity that allows these cells to switch between one another through a biological process known as epithelialmesenchymal transition (EMT). This process involves extensive, transcriptionally regulated phenotypic changes that include the loss of cell-cell adhesion and of cell polarity, and the gain of migratory and invasive properties. EMT has been shown to induce stem cell properties ${ }^{176,177}$, prevent apoptosis and senescence ${ }^{178-180}$, and contribute to immunosuppression ${ }^{181}$. With these qualities, EMT is essential in the development and differentiation of tissues and organs but can negatively contribute to organ fibrosis and the initiation of metastases.

\section{Phenotypic and Genetic Changes}

The first steps of EMT are the disassembly of the epithelial cell-cell junctional complexes. Upon induction of EMT, junctional proteins are relocalized and/or degraded. Tight junctional disruption is characterized by decreased claudin and occludin expression and the dissociation of zonula occludens $1(\mathrm{ZO}-1)$ from the cell periphery. This is accompanied by the down-regulation and subsequent loss of expression of the epithelial adherens junction protein, cadherin (E-cadherin), which is considered a crucial step in the progression of EMT. ${ }^{182}$ This disruption of junctional proteins results in a reorganization of the actin cytoskeleton and loss of apical-basal polarity. The repression of epithelial genes, such as tight junctions, is subsequently followed by the activation of mesenchymal genes like vimentin, fibronectin, and N-cadherin. ${ }^{183}$ These genetic changes associated with EMT are regulated by families of transcription factors including Snail, zinc finger Ebox-binding homeobox (ZEB), and Twist. ${ }^{183}$ When the epithelial actin cytoskeletal 


\section{Lumen}

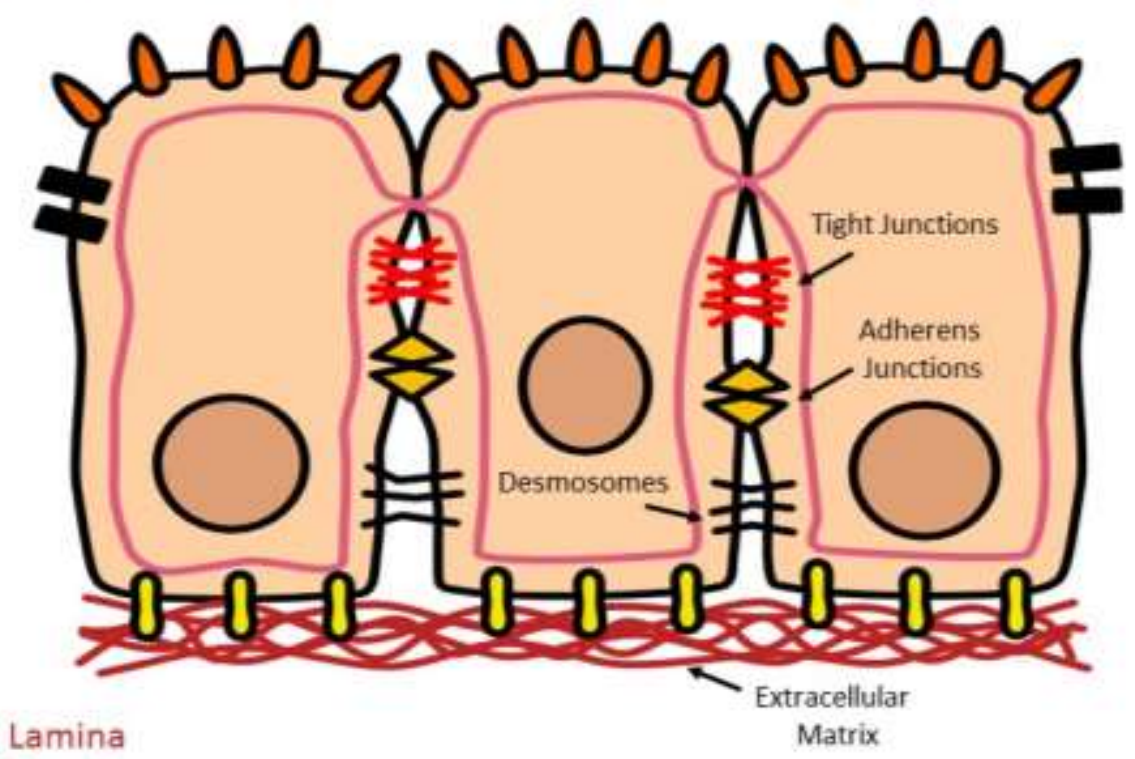

Propria

Figure 2-1. Organization of Cellular Junction Complex.

In the intestinal epithelium, neighboring cells form regulated cellular junction complexes to create an epithelial barrier. From the apical portion of the cell that is exposed to the lumen to the basal extracellular matrix, the cellular junction complex is composed of tight junctions, adherens junctions, and desmosomes. 
structure reorganizes, cells can acquire motility and invasive capacities by forming lamellipodia, filopodia, and invadopodia and by expression matrix metalloproteinases (MMP) that can degrade extracellular matrix proteins. Figure 2-2 gives an overview of the changes that occur during EMT.

\section{Induction and Regulation}

There are several families of transcription factors that can induce EMT, including the zinc finger binding transcription factors Snaill and Snail2, ${ }^{184-186}$ the basic helix-loophelix (bHLH) factors like ZEB $1{ }^{187}$ and ZEB2, ${ }^{188}$ and Twist, ${ }^{189}$ in addition to a T cell factor (TCF) known as lymphoid enhancer binding factor-1 (LEF-1). ${ }^{190,191}$ These EMTinducing transcription factors can be activated by various extracellular stimuli and mediated through a number of signaling pathways including transforming growth factorbeta (TGF- $\beta$ ), bone morphogenetic protein (BMP), epidermal growth factor (EGF), fibroblast growth factor (FGF), Wnt, Hedgehog (HH), Notch, and integrins. Because signals mediated by these pathways are transduced via intracellular kinase cascades, there can be extensive crosstalk between pathways leading to a common endpoint. Once activated, the transcription factors bind to the promoter of cell adhesion genes to block their transcription and initiate EMT.

\section{Transcription Factors}

\section{Snail Family}

Two of the Snail family of transcription factors, Snail1 (Snail) and Snail2 (Slug), are key repressors involved in EMT. Snaill and Snail2 are zinc-finger binding transcription factors and function by sequence-specific binding to the major groove of DNA. ${ }^{192}$ The consensus binding site for Snail proteins, CAGGTG, ${ }^{184,185,193,194}$ is also known as an enhancer box (E-box). This is the same binding motif used by bHLH transcription factors. ${ }^{195}$ This suggests Snail transcription factors may compete with bHLH factors for binding. Once bound to the E-box, the Snail proteins act as transcriptional repressors. The promoter of $C D H 1$, which encodes E-cadherin, contains two adjacent $\mathrm{E}$ box consensus sequences where Snail proteins can bind to repress its transcription. ${ }^{196}$ These transcription factors achieve repression by recruiting both repressor and chromatin remodeling complexes. ${ }^{197-199}$

While E-cadherin is the only identified target of the Snail family, studies have produced other potential genes that may be directly or indirectly regulated. Overexpression of Snail results in the down regulation of other epithelial markers, like desmoplakin, ${ }^{184}$ Muc- $1^{200,201}$ and cytokeratin-18. ${ }^{202}$ In addition, mesenchymal markers like vimentin and fibronectin become upregulated and reorganized. ${ }^{184}$ 

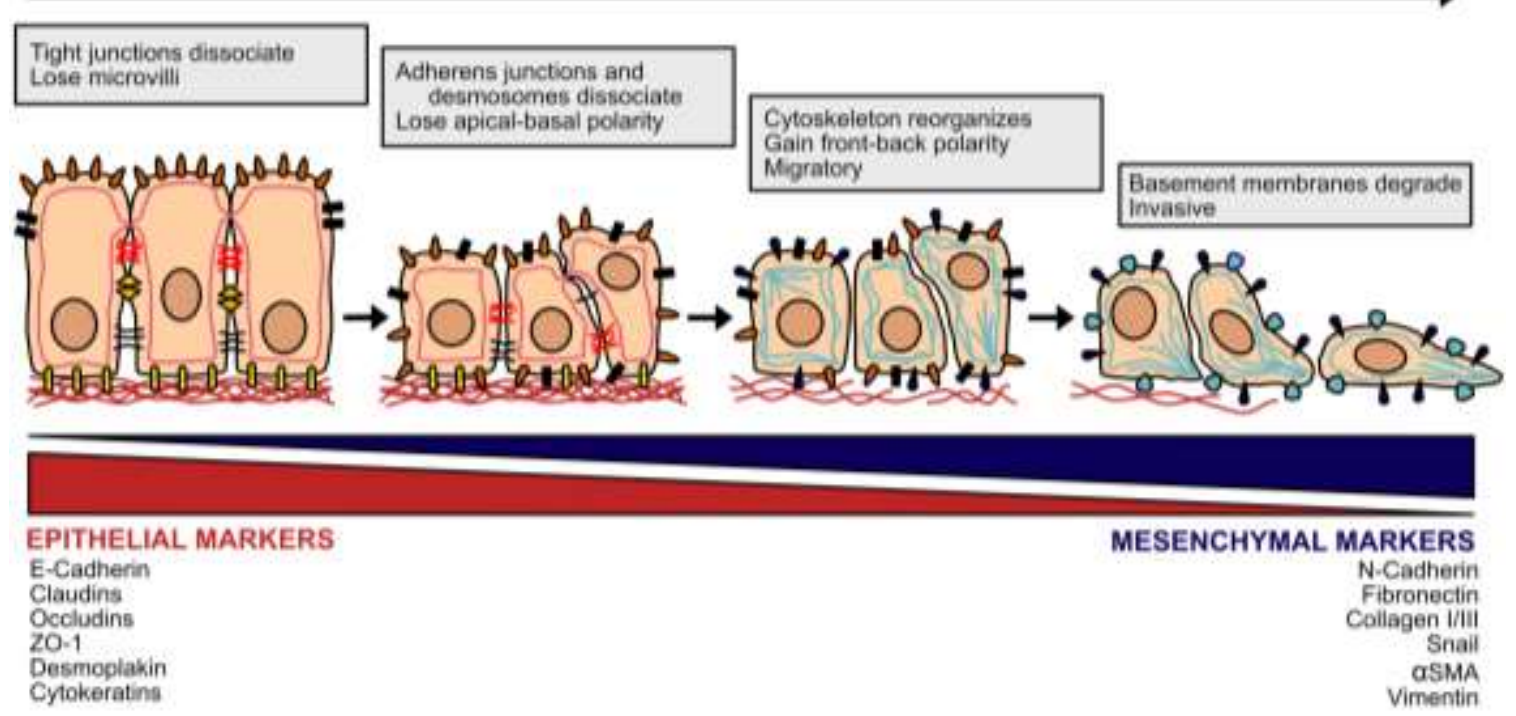

Figure 2-2. Epithelial-Mesenchymal Transition (EMT) Characteristics.

EMT is the biological process in which polarized epithelial cells to undergo genetic and phenotypic in order to switch to mesenchymal cells. This process is characterized by cells losing their cell polarity and cell-cell adhesion and gain migratory and invasive properties common to mesenchymal cells. 


\section{ZEB Family}

The ZEB protein family are bHLH and consist of two members, ZEB1 and ZEB2. Like the Snail family, bind to E-box regions of the $C D H 1$ promoter to repress its transcription. ${ }^{203,204}$ In the nucleus, ZEB proteins mainly interact with activated SMAD proteins and recruit either the corepressor $\mathrm{C}$-terminal binding protein $(\mathrm{CtBP})$ or histone deacetylase complexes. ${ }^{205-208}$ Exogenous ZEB expression in mammary epithelial cells induces the disruption of adherens junctions, through the suppression of E-cadherin, as well as plakophilin-2 and ZO-3. ${ }^{203,204}$ Also, cells transfected with ZEB1 upregulate genes encoding matrix metalloproteinases (MMP), ${ }^{209}$ suggesting ZEB proteins are involved with extracellular matrix (ECM) remodeling associated with EMT.

\section{Twist Family}

Another group of bHLH proteins associated with EMT is the Twist family of transcription factors made up of Twist1 and Twist 2. As with Snail and ZEB, Twist binds to the promoter region of $\mathrm{CDH} 1$ at the $\mathrm{E}$ box regions. There it recruits methyltransferases to facilitate H4K20 monomethylation, which represses the E-cadherin promoter but activates the N-cadherin promoter. ${ }^{210}$ However, this regulation of transcription is not dependent on Twist alone. Twist has been shown to bind the SNAI2 promoter because Snail 2 expression is essential for Twist1-induced invasion and metastasis. ${ }^{211}$

The interplay between Twist and Snaill are also necessary for the induction of ZEB. The transfection of both Twist and Snail are needed for the optimal transcription of ZEB1. ${ }^{212}$ The expression of Snail1 and Twist are also dependent on each other. Down regulation of Twist has been shown to suppress Snail1, and Snaill is necessary for the increase in Twist protein and upregulation of Twist 1 transcript. ${ }^{212}$ These results indicate the co-regulatory function each of these transcription factor families play in the induction of EMT.

\section{Signaling Pathways}

While there are many different extracellular cues inducing EMT, there is significant crosstalk between the signaling cascades. This section highlights the best characterized signaling inducers of EMT (Figure 2-3).

\section{TGF- $\beta$}

TGF- $\beta$ signaling is considered the hallmark inducer of EMT. TGF- $\beta$ is a pleiotropic cytokine involved in cell proliferation, cell differentiation, apoptosis, matrix accumulation and tumor migration. ${ }^{213}$ Signaling is activated by the TGF- $\beta$ superfamily of ligands, which include three isoforms of TGF- $\beta$ (TGF- $\beta 1-3)$ and six isoforms of BMP (BMP-2-7) among other ligands. ${ }^{183}$ TGF- $\beta$ signaling occurs through the binding of a ligand to the tetrameric receptor complex composed of type I and type II TGF- $\beta$ 


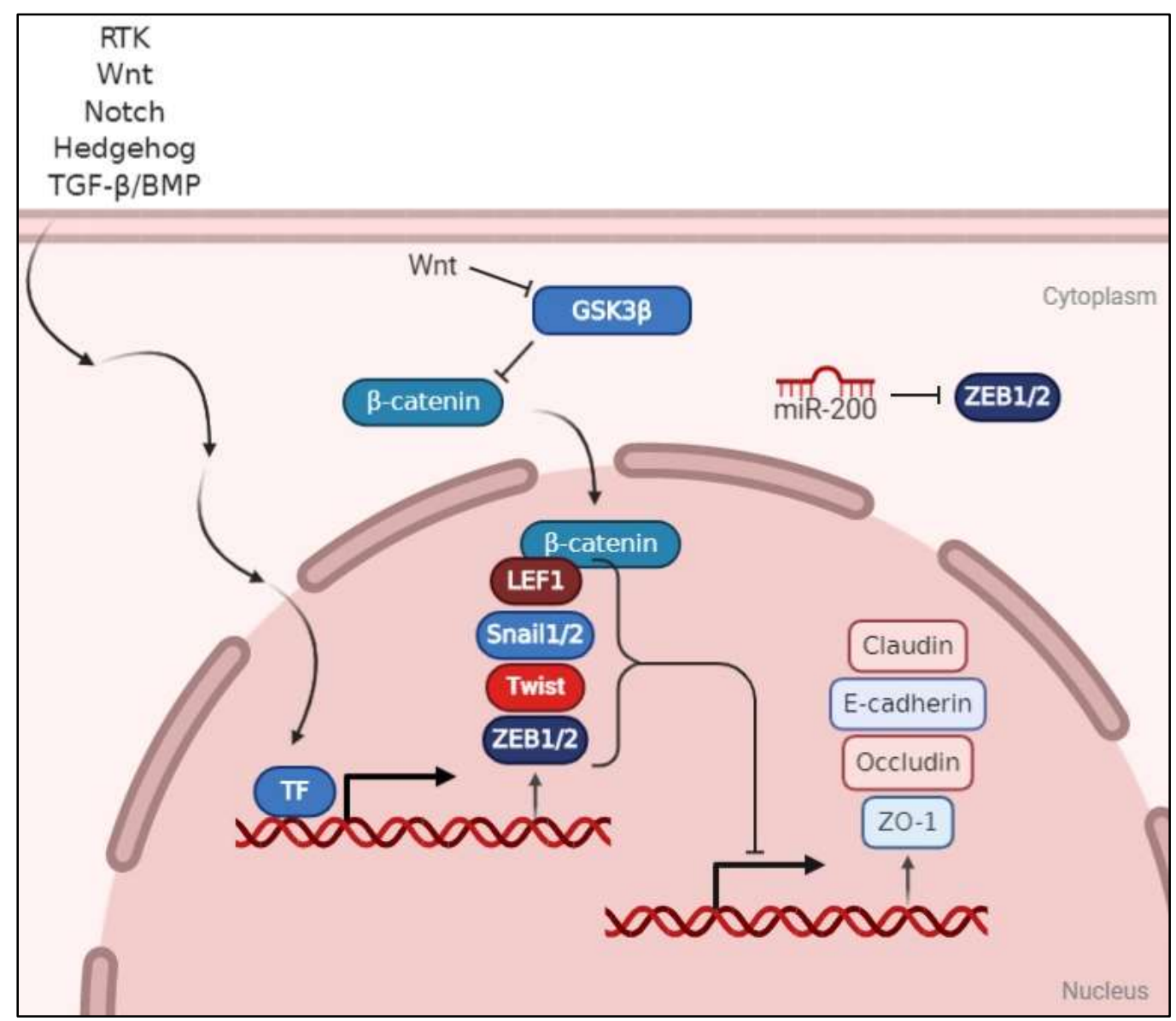

\section{Figure 2-3. EMT Signaling Pathways.}

EMT can be triggered by a number of different extracellular cues that can then be relayed via signaling pathways including transforming growth factor- $\beta$ (TGF- $\beta$ ), bone morphogenic protein (BMP), receptor tyrosine kinase (RTK), Wnt, Notch, and Hedgehog. The result of these signaling cascades is the upregulation of the transcription factors Snail1/2, zinc finger E-box-binding homeobox (ZEB) 1/2, Twist and lymphoid enhancer binding factor-1 (LEF-1). The transcription factors act as transcriptional repressors to induce EMT by inhibiting the transcription of epithelial markers like claudins, E-cadherin, occludin, and zonula occludens-1 (ZO-1). There are known pathways that can inhibit EMT induction, for instance GSK3 $\beta$ targets $\beta$-catenin for degradation, thereby inhibiting it interaction with LEF-1. In addition, the miR-200 family of microRNAs directly inhibits the translation of ZEB. 
receptors. ${ }^{213}$ Once bound, the type II receptor phosphorylates the type I receptor, which in turn phosphorylates an intracellular signaling effector. ${ }^{213}$ Following activation, TGF- $\beta$ induced EMT can proceed through SMAD dependent or independent signaling transduction.

Typically, SMADs are the main intracellular signaling effectors for TGF- $\beta$ signaling transduction. The phosphorylation of the type I TGF- $\beta$ receptor creates a docking site for the receptor regulated SMADs, SMAD2 and SMAD3. The phosphorylation of SMAD2/3 recruits SMAD4, creating the functionally active SMAD complex which can translocate to the nucleus. ${ }^{214}$ Once in the nucleus, the SMAD complex binds the DNA motif CAGAC to transcriptionally regulate downstream genes. ${ }^{215}$ The SMAD complex can bind to the promoter of SNAII to induce the expression of Snail1. Subsequently, Snaill can complex with SMADs to suppress the Snail targets, occludin and E-cadherin. ${ }^{216}$ ZEB transcription factors are also known to bind to SMAD to regulate the expression of Snail1, Snail2 and Twist. ${ }^{217}$

Besides SMAD-dependent signaling, TGF- $\beta$ can activate signaling through Rholike GTPases, which control organization of the actin cytoskeleton and cell mobility. ${ }^{218,219}$ TGF- $\beta$ signaling rapidly activates RhoA and Rho-associated protein kinase (ROCK) in cells undergoing EMT. ${ }^{220-222}$ Conversely, TGF- $\beta$ has been found to down-regulate RhoA as well. Par6, a polarity complex protein, binds to the type 1 TGF- $\beta$ receptor. When the receptor is activated Par6 is phosphorylated and recruits Smurf1, an E3 ubiquitin ligase. ${ }^{223}$ Together the Par6-Smurf1 complex target RhoA for ubiquitination, leading to a loss of cell junctions associated with TGF- $\beta$-induced EMT. ${ }^{223}$ Along with RhoA, TGF- $\beta$ can activate Cdc42, a GTPase highly involved in the formation of lamellipodia and filopodia. ${ }^{224,225}$ In addition to Rho-like GTPases, TGF- $\beta$ receptors have kinase activity and therefore can activate mitogen-activated protein kinase/extrcellular signal regulated kinase (MAPK/ERK), c-Jun N-terminal kinases (JNK)-p38 MAPK, and phosphatidylinositol 3-kinase (PI3K)-Akt. ${ }^{226-234}$ These signaling pathways are similarly activated by the RTKs and so will be described in detail in the next section.

\section{Receptor Tyrosine Kinase}

As the name implies, RTKs are transmembrane-spanning receptors with intracellular C-terminal catalytic domains that have kinase activity. ${ }^{235}$ A number of growth factors and cytokines including epidermal growth factor (EGF), fibroblast growth factor (FGF), insulin-like growth factor (IGF), vascular endothelial growth factor (VEGF), and platelet-derived growth factor (PDGF) are known to signal through RTKs. ${ }^{235}$ As RTK signaling is essential for embryonic development and tissue regeneration and tumorigenesis, it is highly involved in EMT. EGF signaling through MAPK/ERK results in the upregulation of Snail and Twist, the loss of E-cadherin, and the activation of MMPs. ${ }^{236-238}$ In tubular epithelial cells, FGF-2 induces EMT in by upregulating vimentin and fibroblast specific protein-1(FSP-1) and inducing the activity of MMP-2. ${ }^{239}$ In a separate study, FGF-2 was shown to signal via Rho-GTPases and $\mathrm{PI} 3 \mathrm{~K}$ to induce cytoskeletal changes. ${ }^{240}$ IGF has been reported to signal through NF- $\kappa \mathrm{B}$, MAPK/ERK, and PI3K-Akt to induce EMT. ${ }^{241-243} \mathrm{NF}-\kappa \mathrm{B}$ signaling via IGF increases 
Snail expression, ${ }^{241}$ while MAPK/ERK signaling increases ZEB expression. ${ }^{242}$ In breast cancer cells, VEGF inhibits glycogen synthase kinase 3 (GSK3) and induces the upregulation of Snail. ${ }^{244}$ Consequently, Snaill increases the expression of VEGF creating a positive feedback loop. ${ }^{245,246}$ In embryogenesis, PDGF regulates the expression of $\mathrm{CDH} 2$, which encodes $\mathrm{N}$-cadherin, in mesoderm through the PI3K pathway. ${ }^{247}$

\section{Wnt Signaling}

The Wnt signaling cascade begins with Wnt proteins binding to a Frizzled receptor, a family of G-protein coupled receptors (GPCR) and a lipoprotein receptorrelated protein (LRP) co-receptor. ${ }^{248}$ This signaling pathway inhibits glycogen synthase kinase $3 \beta$ (GSK3 $\beta) .{ }^{249}$ In its normal role, GSK3 $\beta$ phosphorylates $\beta$-catenin to mark it for ubiquitination and ultimately degradation. ${ }^{250}$ By inhibiting GSK $3 \beta$, Wnt signaling prevents the degradation of $\beta$-catenin and allows it to regulate gene transcription. In the nucleus, $\beta$-catenin recruits LEF-1 forming a complex that binds to the promoter of $C D H 1$ and suppresses expression of E-cadherin. ${ }^{251}$ The inhibition of GSK3 $\beta$ also has direct effects on Snail by preventing its ubiquitination and consequently promoting EMT associated gene regulation. ${ }^{252}$

Wnt signaling is essential in the development of vertebrates. Canonical WNT signaling is required to generate neural crest precursors, ${ }^{253}$ while Wnt3 knockout mice do not develop proper anterior-posterior neural patterning. ${ }^{254}$ Aberrant Wnt signaling, has also been reported in many different cancer types including prostate, ${ }^{255}$ breast, ${ }^{256}$ colorectal, ${ }^{257}$ and liver cancers. ${ }^{258}$

\section{Notch}

Notch signaling is highly involved in developmental EMT. Upon binding to the Notch receptor by the ligands, Jagged or Delta-like, the Notch intracellular domain (NICD) is cleaved by ADAM-family metalloprotease and $\gamma$-secretase and traffics into the nuclease to regulate gene expression. ${ }^{259}$ The NICD can bind to the SNAI1 promoter to upregulate the expression of Snail1. ${ }^{260}$ In addition, NICD can interact with hypoxiainducible factor $1 \alpha(\mathrm{HIF}-1 \alpha)$ to upregulate lysyl oxidase (LOX), which can then stabilize Snail1 protein. ${ }^{260}$ Interaction of Notch with Snail2 is necessary for Notch-mediated repression of E-cadherin, activation of $\beta$-catenin, and resistance to anoikis. ${ }^{261} \mathrm{In}$ development, Notch and TGF- $\beta$ cooperate to activate Snail 2 in endothelial cells during morphogenesis of the cardiac cushion. ${ }^{262}$ Inhibition of Notch in lung cancer cells showed a partial reversal of EMT and decreased invasiveness. ${ }^{263}$

\section{Hedgehog}

The HH family ligands comprised of Sonic Hedgehog (SHH), Indian Hedgehog, and Desert Hedgehog are important regulators of embryogenesis. These ligands bind to the receptors patched homolog 1 (PTCH1) and PTCH2, However, signaling is not transduced via PTCH receptors, instead binding of $\mathrm{HH}$ to PTCH releases the seventransmembrane receptor Smoothened (Smo) which initiates an intracellular signaling. ${ }^{264}$ 
Smo signaling activates Gli transcription factors, which upregulate target genes such as $P T C H 1$ and $S N A I 1 .{ }^{265}$ Reports have also shown, HH signaling induces TGF- $\beta 1$ secretion to promote motility and invasion of gastric cancer cells. ${ }^{266}$

During development, SHH is expressed in the notochord and the neural tube ${ }^{267}$. It is also required for the survival of myogenic and chondrogenic cell lineages which give rise to the muscle and cartilage tissues. ${ }^{268}$ In rat kidney cells, the exogenous expression of Gli1 upregulates Snaill expression and Ectopic expression of Gli1 in rat kidney epithelial cells induces Snaill expression, and the subsequent the reduction of E-cadherin expression. ${ }^{265}$

\section{MicroRNA}

Recent studies have revealed along with traditional signaling pathways, microRNAs can regulate EMT as well. MicroRNAs (miRNA, miR) are small non-coding RNAs that are approximately 22 nucleotides long. By binding to the 3' untranslated region (UTR) of specific gene transcripts, miRNAs inhibit the translation of that protein (Figure 2-4). ${ }^{269}$ To date, there are more than seventy different miRNAs reported to be involved in EMT regulation. ${ }^{269-271}$ In particular, miRNAs have been shown to target the transcription factors responsible for the induction of EMT. ${ }^{272}$ The miR-220 family members, miR-200a, miR-200b, miR-200c, miR-141, and miR-429, directly target ZEB1/2 to inhibit their expression. ${ }^{273-276}$ In addition, Snail is targeted by members of the miR-30 and miR-34 family as well as miR-203 inhibiting EMT. ${ }^{277-280}$ However, not all miRNAs inhibit EMT, miR-9 is a putative oncogene that directly targets $\mathrm{CDHl}$ thus promoting EMT. ${ }^{281-283}$ Additionally, miR-373 and miR-520c target the cell surface adhesion molecule, CD44, to stimulate tumor invasion. ${ }^{284}$

\section{Types of EMT}

EMT is an expansive topic. To parse out common signaling pathways and transcriptional regulation, researchers have divided EMT into three subtypes.

\section{Type 1: Embryogenesis}

EMT was initially identified in embryos as the fundamental process for the generation of tissues and organs. Type 1 EMT involves primitive epithelial cells transitioning to motile mesenchymal cells as part of gastrulation and generating the neural crest. ${ }^{285}$ During embryogenesis, a fertilized egg will go through gastrulation to generate a primitive streak. ${ }^{286}$ From the primitive streak arises the three primary germ layers that will eventually generate all tissues and organs in the body. In order to form different tissues, cells of the germ layers must undergo programmed genetic and phenotypic changes orchestrated by specific signaling proteins in a process now known 


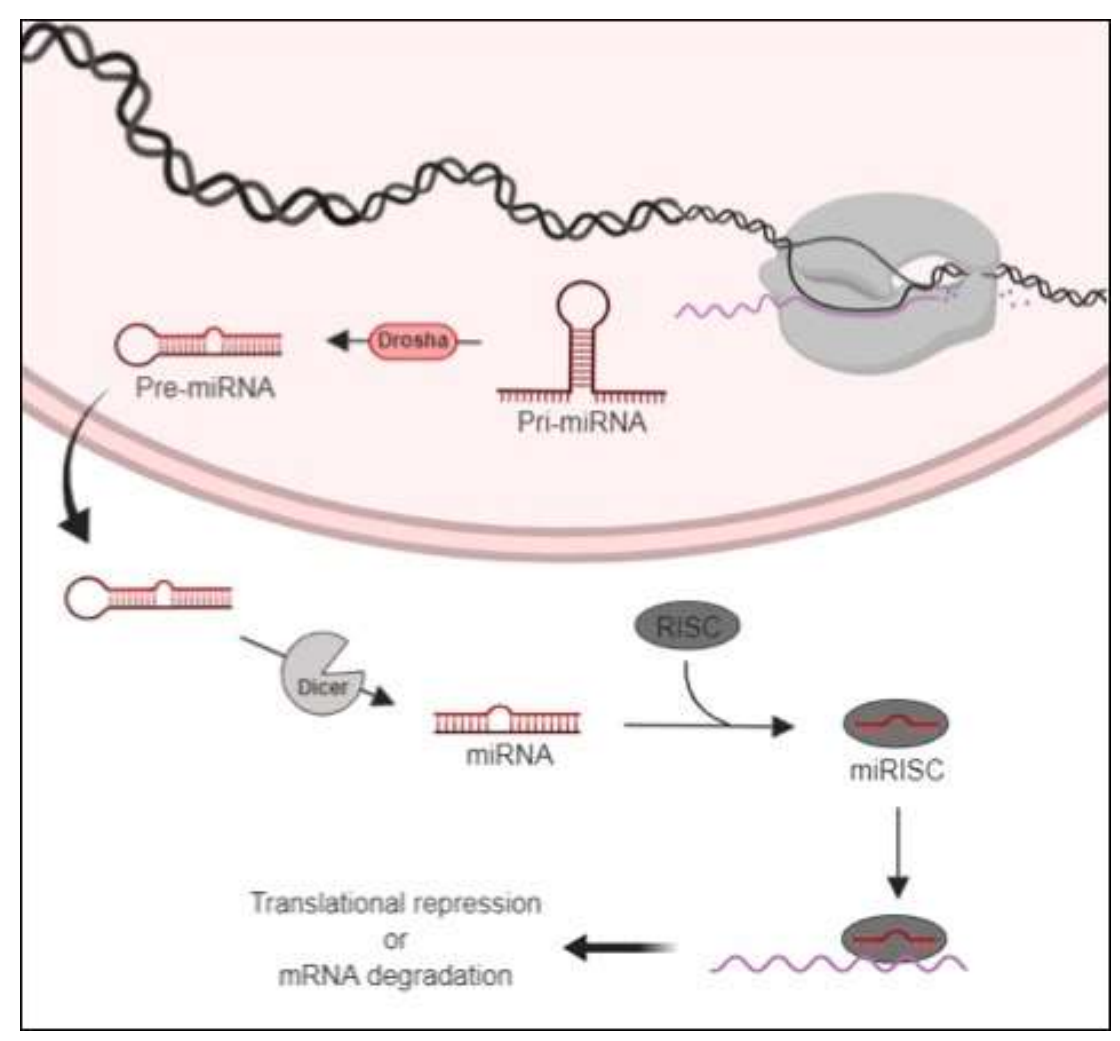

Figure 2-4. MicroRNA Processing and Function.

MicroRNAs (miRNA) are small non-coding RNAs that decrease the expression of mRNAs with sequence complementary to the miRNA. Following transcription, the primRNA are processed by Drosha into a pre-miRNA. Once transported out of the nucleus, the pre-miRNA, is further process by Dicer into mature miRNA. The miRNA can then associate with RISC proteins to bind mRNA, leading to a number of consequences including induced decapping or deadenyltion, altered cap protein binding, reduced ribosome occupancy, and sequestration of the mRNA from translational machinery 
as EMT. ${ }^{175}$ The resulting mesenchymal cells travel to their intended destination and through the reversal process MET, mesenchymal-epithelial transition, begin early organogenesis. ${ }^{285}$

Type 1 EMT is dependent on canonical Wnt, FGF, and BMP signaling. Embryos with Wnt3 knocked out are incapable of undergoing the EMT necessary for gastrulation. ${ }^{254} \mathrm{Wnt} 8 \mathrm{c}$ is also necessary for the formation of the primitive streak, in fact over expression of $\mathrm{Wnt} 8 \mathrm{c}$ leads to the formation of duplicate primitive streaks. ${ }^{287,288} \mathrm{FGF}$ signaling has been shown to work in unison with Wnt signaling, inducing Snail and thus repressing E-cadherin. ${ }^{289}$ In further developmental stages of embryogenesis, BMP signaling induces the migratory state necessary to form the neural crest. ${ }^{290,291}$ Subsequent combinations of signaling pathways and regulatory transcription factors associated with EMT are activated to continue the full development of the growing embryo. ${ }^{292}$

\section{Type 2: Wound Healing}

Following injury or chronic inflammation, tissues undergo regeneration and repair. Type 2 EMT involves the process of epithelial cells switching to fibroblasts in response to damage. This is a key distinction between type 1 and type 2 EMT, as type 1 EMT yields mesenchymal cells, while type 2 EMT results in resident tissue fibroblasts. ${ }^{285}$ Along with wound healing, type 2 EMT can also result in tissue fibrosis. Fibroblasts formed by type 2 EMT can migrate and take up residence in the interstitium where they are primed to respond to inflammation. ${ }^{292}$ Tissue injury results in the release of many growth factors and extracellular cues like TGF- $\beta$, PDGF, FGF-2, collagens, and laminins that can stimulate EMT. ${ }^{239,293,294}$

The importance of TGF- $\beta$ signaling in the development of fibrosis was shown in studies using BMP-7, an antagonist TGF- $\beta .^{295-297}$ BMP-7 can inhibit TGF- $\beta$ - induced EMT and rescue E-cadherin expression. ${ }^{295,298}$ In addition, by administering recombinant BMP-7 to mice with fibrosis, researchers were able to reverse EMT and repair fibrotic tissue with healthy epithelial cells. ${ }^{298}$

\section{Type 3: Cancer}

Given that EMT is associated with enhanced cellular mobility, it is to be expected that tumorigenesis is enabled by EMT. Type 3 EMT involves epithelial cancer cells transitioning to invasive tumor cells in order to circulate through the body and to form secondary metastases via MET. ${ }^{299}$ While EMT appears to drive dissemination of tumor cells, it must be halted before metastatic colonization can take place. ${ }^{300,301}$ Interestingly, many circulating tumor cells express both epithelial and mesenchymal markers, ${ }^{302,303}$ suggesting the EMT process is active during the dissemination of cancer cells. While similar signaling pathways are involved in type 3 EMT as type 1 and $2,,^{292,304}$ it is still unclear what signals are distinct to type 3 EMT. $^{299}$ 
In addition to gaining migratory properties, cancer cells undergoing EMT exhibit more aggressive phenotypes, including resistance to chemotherapeutics, ${ }^{305,306}$ inhibition of senescence, ${ }^{180,307}$ and features of stem cells. ${ }^{179,300}$ It has been reported that following the induction of EMT, cancer cells express CD $44{ }^{\text {high }} \mathrm{CD} 24{ }^{\text {low }}$ which are widely accepted as stem cell markers. ${ }^{176,308,309}$ In a study using human mammary cells, induction of EMT via TGF- $\beta$ treatment or the overexpression of Snail and Twist resulted in the cells exhibiting increased self-renewal and ability to form tumors 301

\section{Partial EMT}

While it is easy to think of EMT and MET as processes with distinct mesenchymal or epithelial outcomes, recent research suggests EMT is really a spectrum with cells displaying both epithelial and mesenchymal characteristics. ${ }^{300,310-312}$ In this phenotype cells may express collagen I, vimentin or $\alpha$-SMA, while also retaining loose cell-cell adhesion proteins. ${ }^{292}$ This so called "metastable" or partial EMT has been reported in association with all 3 types of EMT. ${ }^{313-316}$ Circulating tumor cells exhibiting a hybrid epithelial/mesenchymal phenotype have been shown to migrate and invade the ECM as multicellular clusters. ${ }^{303,317,318}$ Interestingly, in so cases partial EMT seems to be the preferred outcome. In a model of kidney fibrosis, renal epithelial cells do not undergo full EMT, rather they provide signals needed for the differentiation of myofibroblasts. ${ }^{315}$ Also instead of reverting back to epithelial cells, these cells remain in the interstitium poised to respond to further inflammation. ${ }^{315}$ Partial EMT highlights the intricate regulation of transcriptional activators and suppressors necessary for the induction and progression of EMT.

\section{Viral-Induced EMT}

Relatively few viruses are known to induce signatures of EMT. Hepatitis B virus $(\mathrm{HBV})^{319}$, hepatitis C virus $(\mathrm{HCV})^{320}$, human papilloma virus (HPV) $)^{321}$, Epstein-Barr virus (EBV) ${ }^{322}$, Kaposi sarcoma-associated herpes virus (KSHV) $)^{323}$ and cytomegalovirus $(\mathrm{CMV})^{324,325}$ have all been shown to drive EMT, which is not surprising given that they are oncogenic viruses. EMT is thought to initiate metastases and cause hepatocellular carcinoma $^{326}$, cervical carcinomas ${ }^{327}$, and lymphoma ${ }^{326}$ among other diseases associated with infection by these viruses. While the signaling pathways utilized by these viruses to induce EMT are different, it is regulated through the interaction of one or more of their viral proteins. ${ }^{322,323,328-339}$ More recently rhinovirus ${ }^{340}$ and transmitted gastroenteritis coronavirus (TGEV) ${ }^{341}$ were shown to induce EMT. The mechanisms leading to EMT and the role in pathogenesis remains unknown. However, TGF- $\beta$ is implicated as the main signal transducer for both viruses. ${ }^{340,341}$ 


\section{Research Aims}

The overall goal of my studies was to determine the underlying mechanisms(s) of astrovirus-induced EMT and determine the impact of EMT on astrovirus replication. To our knowledge, these are amongst the first studies focused on understanding EMT induced during infection with a non-oncogenic virus and will lead to important new information on viral-regulated EMT as well as a better understanding of astrovirus pathogenesis. I hypothesized that human astrovirus activates TGF- $\beta$ leading to EMT in Caco-2 intestinal epithelial cells. This hypothesis was tested through the following specific aims:

\section{Aim 1: Define the Extent of Astrovirus-Induced EMT}

Through the use of qRT-PCR, Western blotting analysis and confocal microscopy of the EMT associated markers occludin, E-cadherin, Snail, Twist, vimentin, and fibronectin, we characterized the hallmarks of astrovirus-induced EMT in human intestinal epithelial Caco-2 cells. The hallmarks of EMT that were investigated include: the loss of epithelial gene expression and dysregulation of cell junctions, the loss of apical-basal polarity, the activation of mesenchymal genes, and increased expression of mesenchymal proteins.

\section{Aim 2: Examine the Mechanism(s) for Astrovirus-Induced EMT}

Our laboratory has previously shown that the astrovirus capsid protein is sufficient to cause barrier permeability and has more recently found that astrovirus infection increased TGF- $\beta$ activity. The goal of this aim was to determine if astrovirus replication and/or astrovirus-activated TGF- $\beta$ are required for EMT induction

The data formed by this project will not only provide fundamental new research on astrovirus pathogenesis, they will increase our understanding of non-oncogenic induced EMT. The outcome of these studies will likely lead to an exciting new area of investigation for non-oncogenic viruses and novel areas of research into the physiology of the gastrointestinal tract by enteric viruses. 


\section{CHAPTER 3. MATERIALS AND METHODOLOGY ${ }^{1}$}

\section{Cell Culture Procedure}

The human intestinal adenocarcinoma cell line Caco-2 was obtained from ATCC (HTB-37). The cells were propagated in minimum essential medium (MEM; Corning) supplemented with 20\% fetal bovine serum (FBS; HyClone), GlutaMax-I (Gibco), $1 \mathrm{mM}$ sodium pyruvate (Gibco), and penicillin-streptomycin (Gibco).

The mink lung epithelial cells (Mv1Lu) used in the PAI assay were obtained from a collaborator. The cells were propagated in Dulbecco's Modified Eagle Medium (DMEM; Corning) supplemented with 10\% FBS (HyClone) and were maintained under selective pressure with $0.4 \mathrm{mg} / \mathrm{ml}$ of G418 (InvivoGen).

The occludin knockdown Caco-2 cells were a generous gift from Dr. Carolyn Coyne. The cells were propagated in DMEM (Corning) supplemented with $10 \%$ FBS and $0.01 \mathrm{mg} / \mathrm{ml}$ human transferrin (Sigma).

\section{Reagents and Methods Related to HAstV-Induced EMT}

\section{Virus Propagation}

HAstV-1 was propagated in Caco-2 cells, and the titers of the virus were determined on Caco- 2 cells by the fluorescent-focus assay as previously described. ${ }^{343}$ To UV inactivate the virus, $100 \mu \mathrm{l}$ of HAstV-1 was subjected to $100 \mathrm{~mJ} / \mathrm{cm} 2$ with a UV cross-linker as described previously, ${ }^{41}$ and inactivation was confirmed by the fluorescentfocus assay.

\section{Immunofluorescent Staining and Confocal Imaging}

Briefly, Caco-2 cells were seeded onto glass coverslips (for epithelial and vimentin staining) or transwells (polarity and SMAD staining). Once confluent, the cells were infected with HAstV-1 (MOI 10) or mock infected. At various times post-infection, cells were fixed with $4 \%$ paraformaldehyde (for epithelial and vimentin staining) or $100 \%$ ice cold methanol (polarity and SMAD staining), and then blocked with 5\% normal goat serum (NGS) in PBS at room temperature for $1 \mathrm{hr}$. The cells were stained for Ecadherin (33-4000; Invitrogen), occludin (71-1500; Invitrogen), ZO-1 (33-9100 and 617300; Invitrogen), sodium-potassium ATPase (ab167390; abcam), ezrin (MA5-13862;

\footnotetext{
${ }^{1}$ Portions of chapter from previously published article; final submission modified with permission. Hargest V, Sharp B, Livingston B, Cortez V, Schultz-Cherry S. Astrovirus Replication Is Inhibited by Nitazoxanide In Vitro and In Vivo. J. Virol. 94, (2020) doi: 10.1128/JVI.01706-19. ${ }^{342}$
} 
Invitrogen), $\beta$-catenin (ab32572; abcam), SMAD3 (51-1500; Invitrogen), HAstV capsid(8e7; DakoCytomation), and vimentin (ab92547; abcam) for $1 \mathrm{hr}$ followed by antimouse IgG-Alexa Fluor 488 or anti-rabbit IgG-Alexa Fluor 555 (Invitrogen) secondary antibodies and DAPI (4',6'-diamidino-2-phenylindole; Sigma) in 1\% NGS for $30 \mathrm{~min}$ at room temperature. Following staining, coverslips or transwells were mounted onto slides with Prolong Gold Antifade Mountant (Invitrogen) and sealed. Cells were imaged with a Nikon TE2000 inverted microscope Images were captured with a Nikon 60x objective lens using Nikon NIS Elements software.

\section{Western Blotting}

Caco-2 cells were mock infected or HAstV-1 (MOI 10) infected, or were treated with an equal amount of UV-inactivated virus, or mock-infected lysate in MEM as a negative control. At the indicated times, monolayers were lysed in $100 \mu$ of RIPA buffer (Abcam) and $1 \times$ protease inhibitor cocktail (Pierce) for $15 \mathrm{~min}$ at room temperature and centrifuged at $14,000 \times \mathrm{g}$ for $5 \mathrm{~min}$ at $4^{\circ} \mathrm{C}$. Protein concentrations were determined using the BCA Protein Assay Kit (Pierce). Equal protein concentrations of the soluble fraction were separated by sodium dodecyl sulfate-polyacrylamide gel electrophoresis (SDSPAGE) (4-20\%) under reducing conditions. Following transfer to nitrocellulose and probing for for E-cadherin (33-4000; Invitrogen), occludin (71-1500; Invitrogen), ZO-1 (33-9100; Invitrogen), $\beta$-catenin (ab32572; abcam), vimentin (ab92547; abcam), and $\beta$ actin (A5441; Sigma). The blot was imaged on Licor Odyssey Fc and band densitometry was measured using Image Studio version 5.2 software.

\section{RT $^{2}$ Profiler}

Briefly, cells were seeded in 6-well plate, mock infected or HAstV-1 (MOI 10) infected and collected at the indicated time point in TRIzol reagent (Thermo Fisher Scientific). Then, RNA was isolated according to the manufacturer's instructions. RNA quality was determined and was reverse transcribed using Qiagen's RT ${ }^{2}$ First Strand Kit (Cat\# 330401). The cDNA was used on the real-time RT ${ }^{2}$ Profiler PCR Array (Cat\#. PAHS-090Z) in combination with RT ${ }^{2}$ SYBR Green qPCR Mastermix (Cat\# 330529). The $\mathrm{Ct}$ values were then uploaded on to the data analysis web portal at http://www.qiagen.com/geneglobe. Samples were assigned to either control or test groups. The data was normalized based on a manual selection from full panel of reference genes. The data analysis web portal calculated fold change/regulation using $\Delta \Delta \mathrm{Ct}$ method, in which $\Delta \mathrm{Ct}$ is calculated between gene of interest and an average of reference genes (B2M, HPRT1, and RPLP0), followed by $\Delta \Delta \mathrm{Ct}$ calculations ( $\Delta \mathrm{Ct}$ (Test Group)- $\Delta \mathrm{Ct}$ (Control Group)). Fold Change was then calculated using $2^{\wedge}(-\Delta \Delta \mathrm{Ct})$ formula. 


\section{Quantitative RT-PCR}

Caco-2 cells were infected with HAstV-1 or mock infected and RNA extracted at indicated timepoints using TRIzol (AMbion) according to manufacturer's specifications. Then qRT-PCR was performed using the QuantiTect SYBR green kit (Qiagen) primer assays for OCLN (cat\# QT00081844), CDH1 (cat\# QT00080143), SNAI1 (cat\# QT00010010), and VIM1 (cat\# QT00095795). The resulting Ct values were normalized to $G A P D H$ (cat\# QT00079247). The log transformed $\Delta \Delta \mathrm{Ct}$ values are reported as fold changes over untreated.

\section{TGF-ß Activity Assay}

TGF- $\beta$ activity was measured using a luciferase reporter cell line, as previously described. ${ }^{344}$ Briefly, mink lung epithelial cells (Mv1Lu), stably transfected with a luciferase reporter construct downstream of the plasminogen activator inhibitor-1 (PAI-1) promotor, were plated in a 96-well tissue culture plate $\left(2 \times 10^{4}\right)$. These cells were inoculated with supernatants $(100 \mu \mathrm{l})$ taken from HAstV-1 (MOI 10) or mock-infected Caco-2 cells at various times post-infection and incubated at $37^{\circ} \mathrm{C}$ for $16-20$ hours. Active TGF- $\beta$ (R\&D Systems) served as a positive control. The inoculum was removed and the cells washed twice with PBS. Cell lysates were prepared and assayed for luciferase activity using the Luciferase Assay System (Promega) and imaged on the Cytation 5 Cell Imaging Multi-Mode Reader (BioTek).

\section{SB431542 and U0126 Treatment}

Briefly, $5 \times 10^{4}$ cells were seeded into transwells (3074; Corning), and once confluent, transferred into serum free media for at least $1 \mathrm{hr}$. The cells were treated with $10 \mu \mathrm{M}$ SB431542 (Tocris) or U0126 (Promega) $1 \mathrm{hr}$ prior to infection. Then the cells were infected with HAstV-1 (MOI 5), TGF- $\beta$ treated (20ng), or mock infected in serum free media. Following the virus adsorption period of $1 \mathrm{hr}$, the inoculum was removed and fresh media containing $10 \mu \mathrm{M}$ SB431542 or U0126 was replaced.

\section{Statistical Analysis}

Data were analyzed by two-way ANOVA followed by Tukey's multiple comparisons test (RT-PCR), ordinary one-way ANOVA followed by a test for trend (epithelial protein expression), two-tailed student t-test ( $\mathrm{Na} / \mathrm{K}$ ATPase localization), twoway ANOVA followed by Sidak's multiple comparisons test (TGF- $\beta$ Activity), ordinary one-way ANOVA followed by Dunnett's multiple comparisons test (E-cad expression with SB431542, UV-inactivated virus, and U0126, and RT-PCR with SB431542 and UVinactivated virus), using GraphPad Prism version 8. Asterisks show statistical significance as follows: *, $\mathrm{P}<0.05$; **, $\mathrm{P}<0.01$; ***, $\mathrm{P}<0.001$. 


\section{Reagents and Methods Related to Nitazoxanide}

\section{Virus Propagation}

Laboratory-adapted human astrovirus stocks (HAstV-1, HAstV-2, HAstV-6, and HAstV-8) were propagated in Caco-2 cells, and the titers of the viruses were determined on Caco-2 cells by the fluorescent focus assay (focus-forming units [FFU]) as previously described. ${ }^{343}$

Clinical isolates (SJ177.110, SJ60.212, SJ88123.E120, and SJ88027.E259) were derived from remnant fecal samples submitted for clinical diagnostic testing at St. Jude Children's Research Hospital. All samples were deidentified before testing. The St. Jude Institutional Review Board approved this study with a waiver of consent. All isolates were propagated in Caco-2 cells. Briefly, a 10 to $20 \%$ dilution of stool extract, positive for HAstV by real-time reverse transcription-PCR (qRT-PCR), was filtered through a $0.22-\mu \mathrm{m}$ filter. The extract was diluted 1:10 in MEM with $5 \mu \mathrm{g} / \mathrm{ml}$ porcine trypsin before adsorption onto Caco- 2 cell monolayers. Following a 1 -h adsorption period at $37^{\circ} \mathrm{C}$, the inoculum was removed and replaced with MEM containing $10 \mu \mathrm{g} / \mathrm{ml}$ porcine trypsin and $0.3 \%$ bovine serum albumin (BSA). The titers of the viruses were again determined on Caco- 2 cells by the fluorescent focus assay. ${ }^{343}$

TAstV-2 stocks were prepared from intestines collected from infected turkey poults. Briefly, pieces of intestine were suspended in $0.5 \mathrm{ml}$ PBS, homogenized using 2mm zirconium oxide beads (Next Advance) for 4 min (at speed setting 4 in the Next Advance air cooling bullet blender), and pelleted by centrifugation at $12,000 \mathrm{rpm}$ for 5 min. The supernatant was pooled and filtered through a $0.2-\mu \mathrm{m}$ filter (fecal filtrate), and viral copy number was quantified by qRT-PCR.

\section{In Vitro HAstV Infection and NTZ Administration}

Briefly, $5 \times 10^{4}$ cells were seeded into 96-well tissue culture plates (Corning), and after 2 days, the cells were inoculated with virus (HAstV-1 or clinical isolates) in serumfree MEM for $1 \mathrm{~h}$ at $37^{\circ} \mathrm{C}$, at which time the virus was replaced with MEM containing $0.3 \%$ BSA and infection was allowed to proceed until 24 hpi or as described in the figure legends. For NTZ treatment, NTZ or DMSO (0.3\% to 3\%) was added to the infection medium at the concentrations and times described in the figure legends.

\section{Immunofluorescent Staining}

Cells were fixed with $100 \%$ ice-cold methanol for $15 \mathrm{~min}$ and then blocked with $5 \%$ normal goat serum (NGS; Gibco) in PBS at room temperature. Cells stained for astrovirus capsid protein were incubated with HAstV mouse monoclonal antibody 8E7 ( $2 \mu \mathrm{g} / \mathrm{ml}$; DakoCytomation), and cells stained for dsRNA were incubated with J2 mouse 
monoclonal antibody (Scicons) ${ }^{345}$ for $1 \mathrm{~h}$ at room temperature. Following incubation with primary antibody, cells were incubated with anti-mouse IgG labeled with Alexa Fluor 488 (Invitrogen) as the secondary antibody and with DAPI (4',6'-diamidino-2-

phenylindole; Sigma) for $30 \mathrm{~min}$ at room temperature. Staining was imaged on the EVOS FL cell imaging system and analyzed using ImageJ 1.50i software.

\section{MTT Cell Viability Assay}

Cell viability was tested using an MTT [3-(4,5-dimethyl-2-thiazolyl)-2,5diphenyl-2H-tetrazolium bromide] cell proliferation assay kit (Abcam) according to the manufacturer's protocol. Briefly, cells were treated with various concentrations of nitazoxanide in serum-free medium for $24 \mathrm{~h}$. The nitazoxanide-containing medium was removed and replaced with a 50:50 mixture of MTT reagent and serum-free medium. The cells were incubated with the mixture at $37^{\circ} \mathrm{C}$ for $3 \mathrm{~h}$. Following incubation, an MTT solvent solution was added and the plate was placed on an orbital shaker for $15 \mathrm{~min}$. The absorbance was then measured as optical density at $595 \mathrm{~nm}$ (OD595). Cell viability was calculated as the percentage compared to the cell viability in nontreated cells.

\section{IFN qRT-PCR}

Caco-2 cells were treated with $2.5 \mu \mathrm{M}$ NTZ or not treated, and RNA extracted at 2 and $4 \mathrm{~h}$ posttreatment using TRIzol (Ambion) according to the manufacturer's specifications. Then, qRT-PCR was performed using the QuantiTect SYBR green kit (Qiagen) primer assays for IFN $\alpha 1$ (catalog number QT00201964; Qiagen), IFN $\beta 1$ (catalog number QT00203763; Qiagen), and IFN $\lambda 1$ (catalog number QT00222495; Qiagen). The resulting cycle threshold $(\mathrm{Ct})$ values were normalized to the value for GAPDH (glyceraldehyde-3-phosphate dehydrogenase) (catalog number QT00079247; Qiagen). The log-transformed $\Delta \Delta \mathrm{Ct}$ values are reported as fold changes over the values for untreated cells.

\section{Animals and NTZ Treatment}

Broad-breasted white turkey poults were obtained from a commercial hatchery. Five-day-old poults were randomly assigned to groups ( $n=6$ per group) and housed in individual, temperature-controlled Horsfall units with HEPA-filtered inlet and exhaust air valves, where they were given free access to water and routine turkey starter feed. Poults were orally inoculated with $500 \mu \mathrm{l}$ of TAstV-2 intestinal filtrate, containing approximately $10^{12}$ to $10^{13}$ genome copies, or PBS alone. Stool samples from individual birds were scored from 1 to 4 . Scoring was performed daily post-infection. Scores of 3 (liquid or loose stool with some undigested food or solid material) and 4 (watery stool with no solids present) were defined as diarrhea, in accordance with previously published work from Meliopoulos et al. ${ }^{42}$ 
For NTZ treatment, poults were orally administered $100 \mathrm{mg} / \mathrm{kg}$ nitazoxanide in $500 \mu \mathrm{l}$ of ultrapure water. Administration of NTZ was carried out for 4 days prior to infection and 3 days post-infection.

\section{Turkey Astrovirus qRT-PCR Assay}

TAstV-2 genome copies were determined as previously described. ${ }^{42}$ Briefly, viral RNA was isolated from 10\% stool by using the MagMAX-96 avian influenza (AI)/Newcastle disease (ND) viral RNA isolation kit (Applied Biosystems) according to the manufacturer's protocol. PCR was performed on $3 \mu$ l of each sample using TaqMan fast virus 1-step master mix (Applied Biosciences) with $600 \mathrm{nM}$ forward primer 5'GACTGAAATAAGGTCTGCACAGGT, $600 \mathrm{nM}$ reverse primer 5'AACCTGCGAACCCTGCG, and $200 \mathrm{nM}$ probe 6-carboxyfluorescein (6FAM)ATGGACCCCCTTTTTCGGCGG-BHQ1 (black hole quencher 1) under the following conditions: $50^{\circ} \mathrm{C}$ for $5 \mathrm{~min}$ and $95^{\circ} \mathrm{C}$ for $20 \mathrm{~s}$, followed by 45 cycles with one cycle consisting of $95^{\circ} \mathrm{C}$ for $3 \mathrm{~s}$ and $60^{\circ} \mathrm{C}$ for $30 \mathrm{~s}$ on a Bio-Rad CFX96 real-time PCR detection system. The number of genome copies/ $\mu$ l of total RNA was determined using a standard curve generated from a synthesized TAstV-2 DNA sequence, comprising nucleotides 4001 to 4201, with a known copy number (calculated using Thermo Fisher Scientific DNA Copy Number and Dilution Calculator). Log10 dilutions of the synthesized TAstV-2 DNA were used for qRT-PCR as described above.

\section{Statistical Analysis}

Data were analyzed by ordinary one-way analysis of variance (ANOVA) (EC 50 and MTT assay), multiple t test (capsid and dsRNA staining time course), and two-way ANOVA (turkey stool titers) using GraphPad Prism version 8.

\section{Transepithelial Electrical Resistance (TER)}

Caco- 2 cells were plated at a density of $5 \times 10^{4}$ cells/well on $6.5 \mathrm{~mm}$ semipermeable $(0.4 \mu \mathrm{m})$ tissue culture inserts $(3470$; Corning) and incubated for 3 or 4 days until the TER reached approximately $1,000 \Omega \cdot \mathrm{cm}^{2}$. Cells were then transferred to serum free media at least $1 \mathrm{hr}$ prior to treatment. The cells were treated with UVinactivates supernatant or infected with HAstV-1 (MOI 10), and TER levels were measured continuously every 15 min for 24 hours using a cellZscope + voltohmmeter (nanoAnalytics). Results are presented as percentages of the insert's initial TER reading (time zero) relative to mock infection. 


\section{Astrovirus Capsid ELISA}

96-well ELISA plates (9018; Corning) were coated with $5 \mathrm{ug} / \mathrm{ml}$ HAstV capsid protein, purified whole HAstV-VA1 virus or BSA in PBS. The coated plates were allowed to incubate at $4{ }^{\circ} \mathrm{C}$ overnight. The plates were then washed $3 \mathrm{x}$ with at least $200 \mathrm{ul}$ PBS-0.01\% tween (PBST). Following washing, the plates were blocked with 4\% BSA in PBST for $1 \mathrm{hr}$ at room temperature. After blocking, the plates were washed as before and then sequential dilutions of IVIG were plated in 1\% BSA in PBST. This was allowed to incubate 1-2 hr at room temperature. Following incubation, any free IVIG was washed away as previously described. Then anti-human secondary antibody conjugated to HRP at a dilution of 1:20000 1\%BSA in PBST was added to the plate and allowed to incubate 1 $\mathrm{hr}$ at room temperature. The plate was washed again and R\&D substrate detection reagent (DY999) was added. The plate was allowed to incubate 5-10 min and the reaction was stopped with $2 \mathrm{~N} \mathrm{H}_{2} \mathrm{SO}_{4}$. The plate absorbance was read at $450 \mathrm{~nm}$ and $570 \mathrm{~nm}$. To calculate the final O.D., the reading at $570 \mathrm{~nm}$ was subtracted from the reading at 450 $\mathrm{nm}$, followed subtracting the reading for each sample on the BSA coated plate to account for non-specific binding. A positive reading was considered any O.D. $3 x$ that of a secondary only control well.

\section{TGF- $\beta$ Activation}

Conditioned media containing latent TGF- $\beta$ from Caco- 2 cells was treated with heat $\left(100^{\circ} \mathrm{C}\right.$ for $\left.10 \mathrm{~min}\right), 1 \mathrm{~N} \mathrm{HCl}$, thrombospondin-1 (TSP $\left.-1 ; 4 \mu \mathrm{g} / \mathrm{ml}\right)$, or purified HAstV-1 (10\% by volume). Excluding the heat treatment, all samples were allowed to incubate for $1 \mathrm{hr}$. Following incubation, the $\mathrm{HCl}$ was neutralized with $1 \mathrm{~N} \mathrm{NaOH}$. Then all samples were assayed for active TGF- $\beta$ using the PAI assay as previously described.

\section{Neutralization Assay}

HAstV-VA1 was pre-incubated with dilutions (1:50 and 1:100) of IVIG for $1 \mathrm{hr}$ at room temperature. Following incubation, Caco-2 cells were infected (MOI 1) with the virus-antibody mixture. After a $1 \mathrm{hr}$ adsorption period, the virus-antibody mixture was removed and fresh media was replaced. At 5 days post-infection, RNA was extracted from supernatants and cell lysates using Qiagen's Viral RNA Extraction Kit. Quantitative RT-PCR was performed according to the previously published protocol for HAstVVA $1 .{ }^{68}$ 


\section{CHAPTER 4. ASTROVIRUS INDUCES REPLICATION-DEPENDENT EPITHELIAL-MESENCHYMAL TRANSITION IN CACO-2 INTESTINAL EPITHELIAL CELLS}

\section{Introduction}

Human astroviruses (HAstV) are small, non-enveloped positive-sense singlestranded RNA viruses in the Astroviridae family. They are a leading cause of diarrhea in children, the elderly, and immunocompromised people. Around $90 \%$ of the population ages 9 years and older have detectable antibodies to HAstV-1. ${ }^{346}$ Despite its prevalence, ${ }^{97,152,155}$ astrovirus remains under studied due to its perceived low clinical impact and our knowledge surrounding astrovirus pathogenesis including the mechanism used to cause diarrhea is limited.

Epithelial cells, the main cell type infected by HAstVs, line the intestinal lumen and function as a barrier that absorbs nutrients and electrolytes while restricting entry of harmful substances or pathogens. ${ }^{347}$ Breaches in this barrier by death of the epithelial cells or disruption of cellular junctions through non-cytopathic mechanisms or inflammation are associated with gastrointestinal diseases including irritable bowel syndrome, Crohn's disease, and colitis. ${ }^{348}$ We have demonstrated that astrovirus disrupts the intestinal barrier through a novel mechanism independent of cellular damage or induction of the host inflammatory response. ${ }^{39,41}$ Instead, astroviruses increase barrier permeability by dysregulation of cellular junctions. ${ }^{349}$ This disruption of barrier function does not require productive infection; the viral capsid protein alone is sufficient to cause disruption in vivo and in vitro. ${ }^{349,350}$ Following gene set enrichment analysis of HAstV-1infected and uninfected Caco-2 cells, we observed that the hallmark pathway of epithelial-mesenchymal transition (EMT) was significantly upregulated. We hypothesize that the reorganization of junctional proteins was the first sign of a much bigger process and that HAstV infected cells are undergoing EMT.

During EMT, polarized epithelial cells undergo changes to assume a more invasive and mesenchymal phenotype. Part of this complex process is the reprogramming of gene expression, regulated by numerous signaling pathways. Activation of these signaling pathways ultimately results in the upregulation of EMT associated transcription factors Snail1/2, Twist, and ZEB1/2 ${ }^{351}$ among others. These transcription factors negatively regulate epithelial markers such as occludin, claudins, and E-cadherin, while positively regulating mesenchymal genes like $\mathrm{N}$-cadherin, fibronectin, and vimentin. ${ }^{183}$ These changes at the genetic level allow for the hallmark phenotypic changes associated with EMT like the disassembly of the epithelial cell-cell junctions, the loss of apicalbasal polarity, and the formation of lamellipodia or filopodia to enable migration and invasion. ${ }^{175}$ One such regulator of EMT is transforming growth factor-beta (TGF- $\beta$ ). ${ }^{351-}$ ${ }^{353}$ When activated via phosphorylation by the TGF- $\beta$ type 1 receptor kinases, SMADs dimerize and translocate to the nucleus where they directly bind to DNA or transcription factors, inducing or repressing EMT related genes. ${ }^{213,353}$ Previously we have shown TGF$\beta$ activity is increased during TAstV-2 infection in turkey poults. ${ }^{39}$ 
Activation of signaling pathways initiating EMT can come from a number of different extracellular cues including from pathogens. To date, only a handful of viruses are known to induce EMT: hepatitis B virus (HBV), ${ }^{354}$ hepatitis $\mathrm{C}$ virus (HCV), ${ }^{355}$ human papilloma virus (HPV), ${ }^{356}$ Epstein-Barr virus (EBV), ${ }^{357}$ and cytomegalovirus (CMV). ${ }^{358,359}$ These viruses are oncogenic, and EMT is thought be a mechanism by which they cause metastases, hepatocellular carcinoma, cervical carcinomas, and lymphoma among other diseases. More recently, rhinovirus ${ }^{360,361}$ and transmitted gastroenteritis coronavirus (TGEV), ${ }^{341}$ were shown to induce EMT yet the role in those viral pathogeneses remains unknown. Here we characterize HAstV-induced EMT. This process beginnings with the dysregulation of cellular junctions and an upregulation of EMT related genes at 8 hours post-infection (hpi). In addition, we show disruption of cell polarity and gain of vimentin by $24 \mathrm{hpi}$. While TGF- $\beta$ is activated during HAstV infection, it does not appear to play a role in early EMT induction. To our knowledge, these studies are amongst the first focused on understanding virally-regulated EMT that is unassociated with oncogenesis.

\section{Results}

\section{HAstV Infection Leads to Time-Dependent Reorganization and Decrease of Junctional Protein Expression}

To determine the extent of junctional disruption during HAstV infection, Caco-2 grown on glass coverslips were infected with HAstV-1 and stained for tight junction proteins (occludin and ZO-1) and adherens junction proteins (E-cadherin and $\beta$-catenin) at $6,12,18$, and 24 hpi. While mock-infected cells showed normal cell junction morphology, HAstV-infected cells have disrupted junctional proteins. This disruption was seen as early as 6hpi with occludin beginning to re-localize away from the cell periphery (Figure 4-1). Cellular junction disruption was followed by ZO-1 re-localizing from the cell membrane at $18 \mathrm{hpi}$. The most striking finding was the reorganization of Ecadherin, a key marker of epithelial cells, ${ }^{362}$ from infected cells. E-cadherin is crucial in the establishment and maintenance of the cellular junction complex as a whole, ${ }^{363,364}$ and aberrant expression of E-cadherin is a hallmark of epithelial dysregulation. ${ }^{351}$ This disassembly of epithelial cell-cell contacts, especially the disruption of E-cadherin, during HAstV-1 infection suggested infected cells are undergoing EMT.

\section{HAstV Infection Leads to EMT-Associated Gene Modulation}

EMT is a transcriptionally regulated process. To establish HAstV-1 infected cells were transitioning, we examined mRNA levels of EMT associated genes at 8,24 and 48 hpi by multiplexed qRT-PCR using Qiagen's RT ${ }^{2}$ Profiler system (Figure 4-2). A number of EMT pathways associated with the induction of EMT were upregulated at 


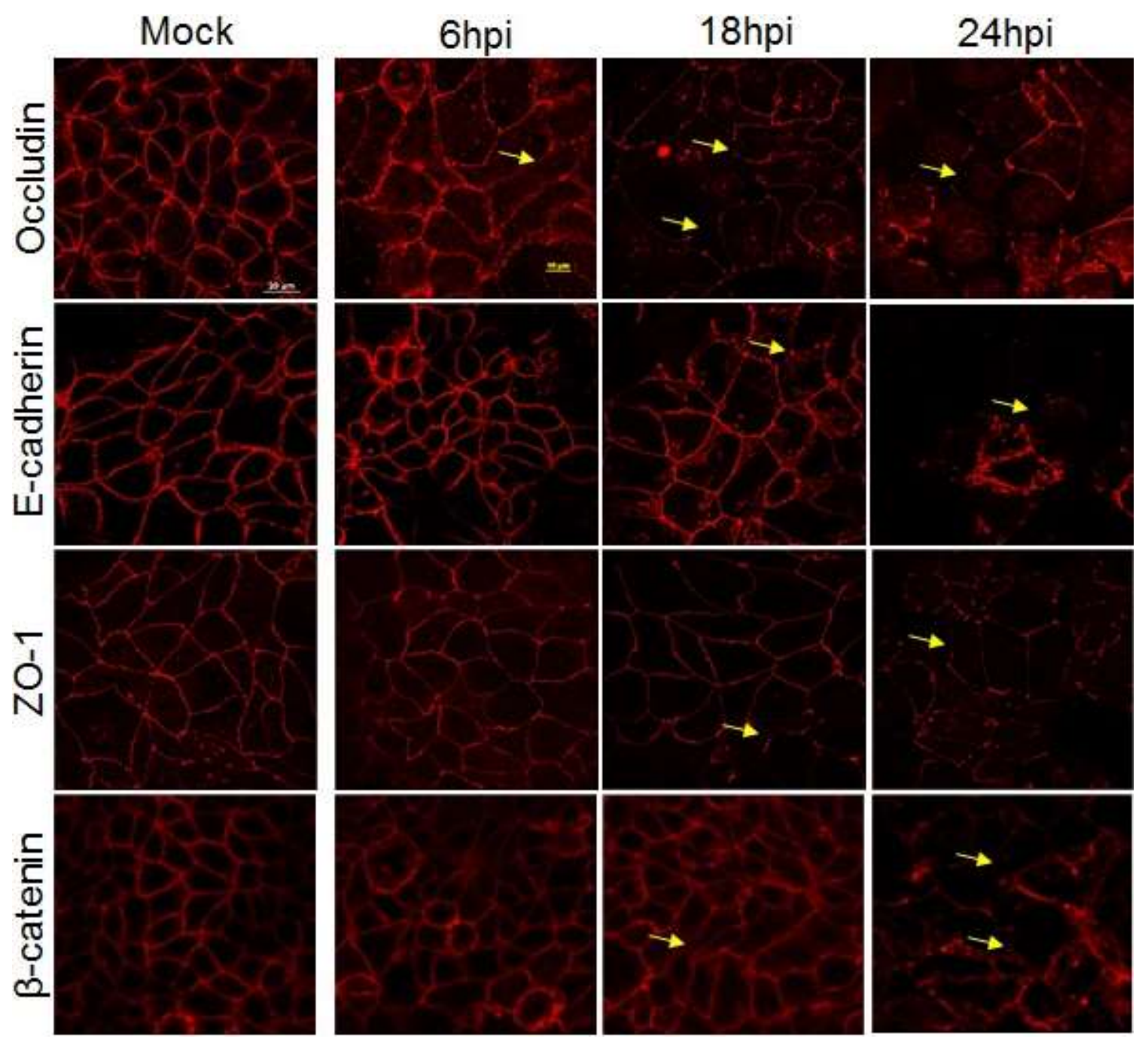

Figure 4-1. HAstV-1 Infection Leads to a Time-Dependent Reorganization and Decrease in Junctional Protein Levels.

Caco-2 monolayers on grown coverslips, infected with HAstV-1 (MOI 10) or mock infected. Cells were fixed at 6,18, and 24 hpi in 4\% paraformaldehyde and then stained for the indicated junctional proteins. Arrows indicate areas of junctional disruption. Images are representative of at least 3 independent experiments. 


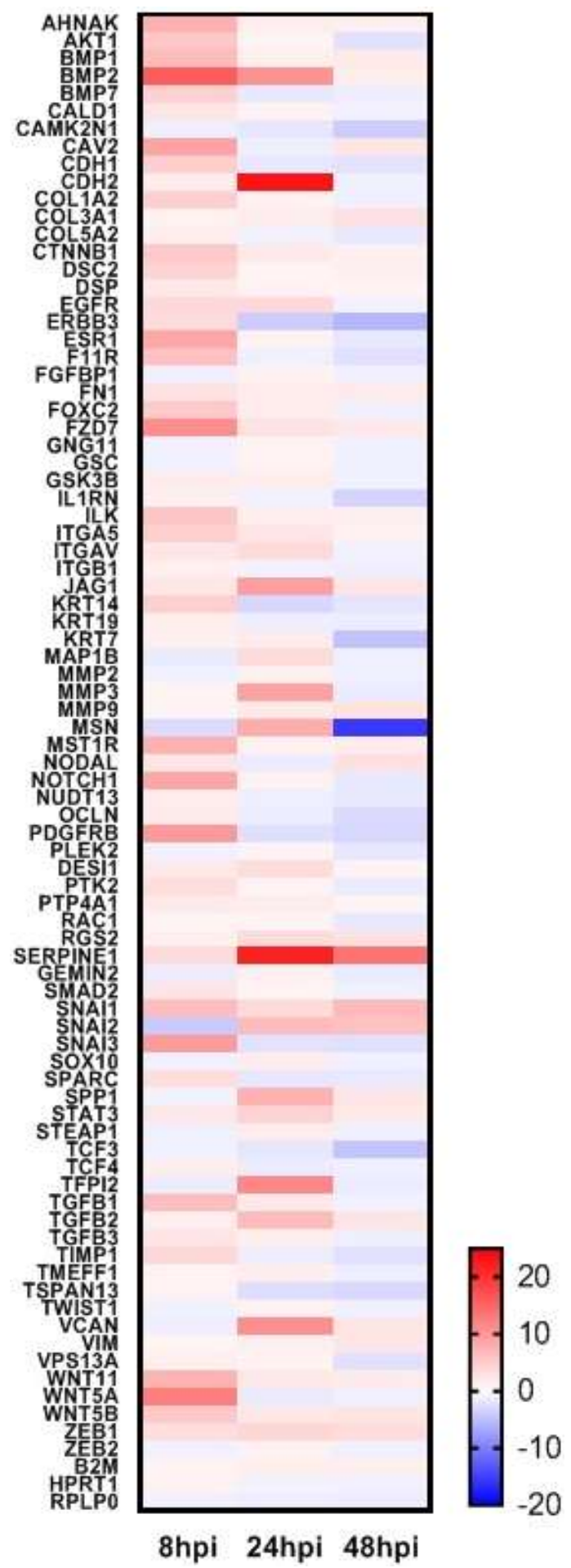

Figure 4-2. EMT-Associated Genes Modulated by HAstV Infection.

Heatmap showing fold regulation of EMT associated genes from Qiagen's RT $^{2}$ Profiler PCR Array Human Epithelial to Mesenchymal Transition (EMT). RNA samples were collected from HAstV-infected or mock-infected cells at 8, 24, and 48 hpi. Gene expression values are colored corresponding to the up (red) or down regulation (blue). 
8hpi including, Wnt, TGF- $\beta$, Notch, and Akt. We validated the RT ${ }^{2}$ Profiler findings by measuring mRNA levels of E-cadherin (CDH1), occludin $(O C L N)$, Snail ( $S N A I 1)$, and vimentin (VIM) by RT-PCR. The epithelial genes, $C D H 1$ and OCLN, were downregulated as early as 8 hpi (Figure 4-3A). The mesenchymal genes SNAII and VIM were strongly upregulated by 18 and $24 \mathrm{hpi}$, respectively. Transcriptional changes were reflected at the protein level, suggesting and that the junctional proteins were not simply reorganized but were downregulated. Over the course of 24 hours, expression of the junctional proteins occludin $(p<0.0001)$, E-cadherin $(p<0.0001), Z O-1(p=0.0058)$, and $\beta$ catenin $(p=0.0139)$ were all significantly decreased (Figure 4-3B, C). Additionally, we observed an increase vimentin staining correlating to the increase in vimentin mRNA (Figure 4-3D).

\section{HAstV-Induced EMT Disrupts Cellular Polarity}

Cellular junctions act as a physical barrier that prevent the movement of lipids and membrane proteins from the apical cell membrane to the basolateral membrane and vice versa. ${ }^{365}$ When cellular junctions are disassembled, proteins that were once localized to the basolateral membrane freely migrate to the apical side causing a loss of cellular polarity. ${ }^{366}$ To determine if cellular polarity was disrupted during HAstV-1 infection, we stained for ezrin, a cytoplasmic linker between the apical membrane and the actin cytoskeleton, ${ }^{367}$ and sodium-potassium ATPase (Na/K-ATPase), a transporter localized to the basolateral membrane. ${ }^{368}$ In mock infected cells ezrin was distinctly localized to the apical side and $\mathrm{Na} / \mathrm{K}$-ATPase to the basolateral with very little overlap (Figure 4-4A). However, by 24 hpi there was less organized arrangement for both proteins and significant co-localization. We noticed that cells appeared to lift or be extruded from the cell monolayer (Figure 4-4A, bottom panel). To quantitate the disruption of polarity, we measured the amount of Na/K-ATPase at the apical membrane. At 24 hpi, there was significantly more $\mathrm{Na} / \mathrm{K}$-ATPase located at the apical membrane than in mock-infected cells (Figure 4-4B, C).

\section{TGF- $\beta$ Activity Is Increased during HAstV Infection}

We next asked what viral and/or cellular factors were involved in HAstV-induced EMT. When examining the genes upregulated early in HAstV infection prior to the induction of EMT, we observed that TGF- $\beta$ mRNA was increased at both 8 and 24 hpi. Since TGF- $\beta$ is a classical activator of EMT, we asked whether this increase in message translated to an increase in TGF- $\beta$ activity using a specific biological reporter assay where mink lung (Mv1Lu) epithelial cells stably expressing the PAI-promoter upstream of luciferase. ${ }^{344}$ Supernatants collected from HAstV-1 infected Caco-2 cells between 4 and $24 \mathrm{hpi}$ were added to the Mv1Lu-PAI cells and TGF- $\beta$ activity was quantitated. Supernatants from HAstV-1- infected cells contained significantly more active TGF- $\beta$ compared to mock-infected cells beginning at 6 hpi and peaking at 12 hpi (Figure 4-5A). Of interest, staining HAstV-1 infected Caco-2 cells for SMADs, the primary signal 
Figure 4-3. HAstV-1 Infection Leads to a Decrease in Epithelial Markers while Increasing Mesenchymal Markers.

(A) Specific EMT genes of interest show epithelial genes (OCLN and CDH1) are down regulated and mesenchymal genes (SNAII and VIM) are upregulated during the course of HAstV-1 infection. Error bars indicate standard deviations, and asterisks show statistical significance as measured by two-way ANOVA followed by Tukey's multiple comparisons test as follows: *, $\mathrm{P}<0.05$; **, $\mathrm{P}<0.01$; ***, $\mathrm{P}<0.001$. (B) Expression of epithelial markers, occludin (Ocln), E-cadherin (E-cad), ZO-1, and $\beta$-catenin ( $\beta$-cat), were quantified by immunoblot of HAst $\mathrm{V}-1$ or mock infected Caco-2 cell lysates. (C) Bands were then quantified by densitometry and normalized to $\beta$-actin then compared to mock-infection. Error bars indicate standard deviations, $p$-value as measured by ordinary one-way ANOVA followed by a test for trend is indicated for each protein. (D) Caco-2 monolayers infected and fixed as previously described and stained for vimentin. 

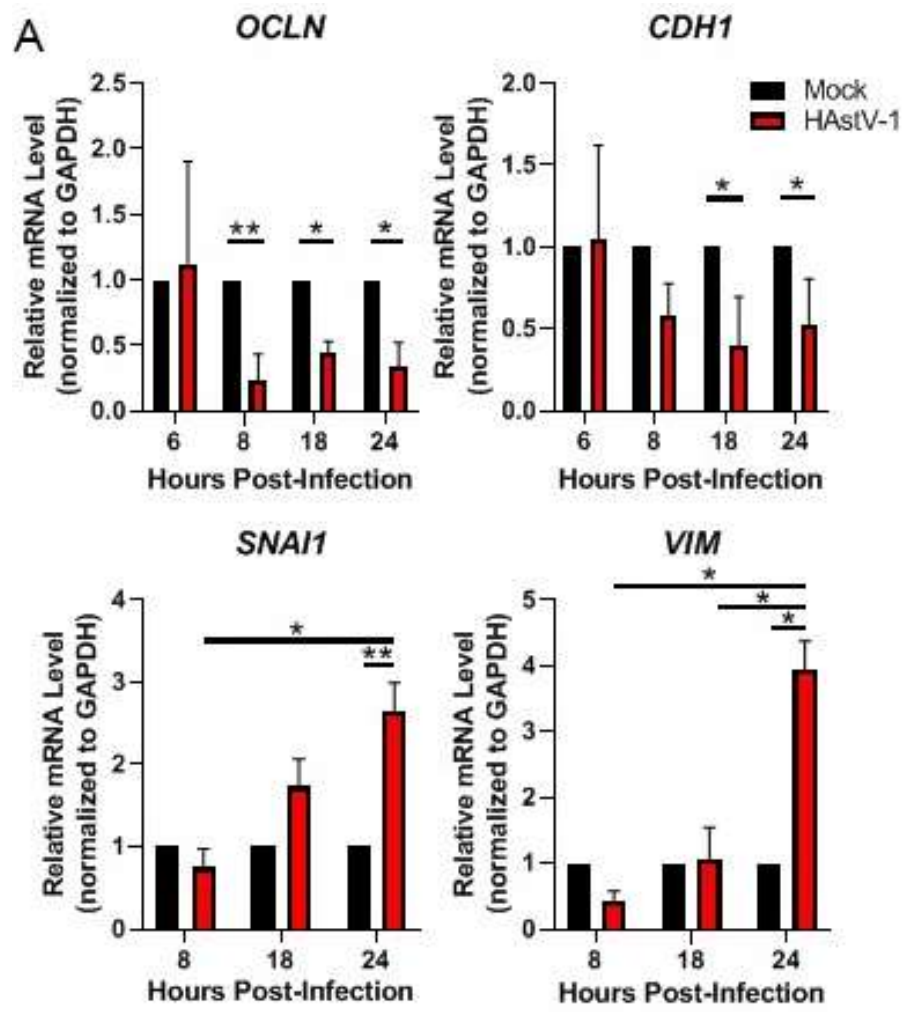

B
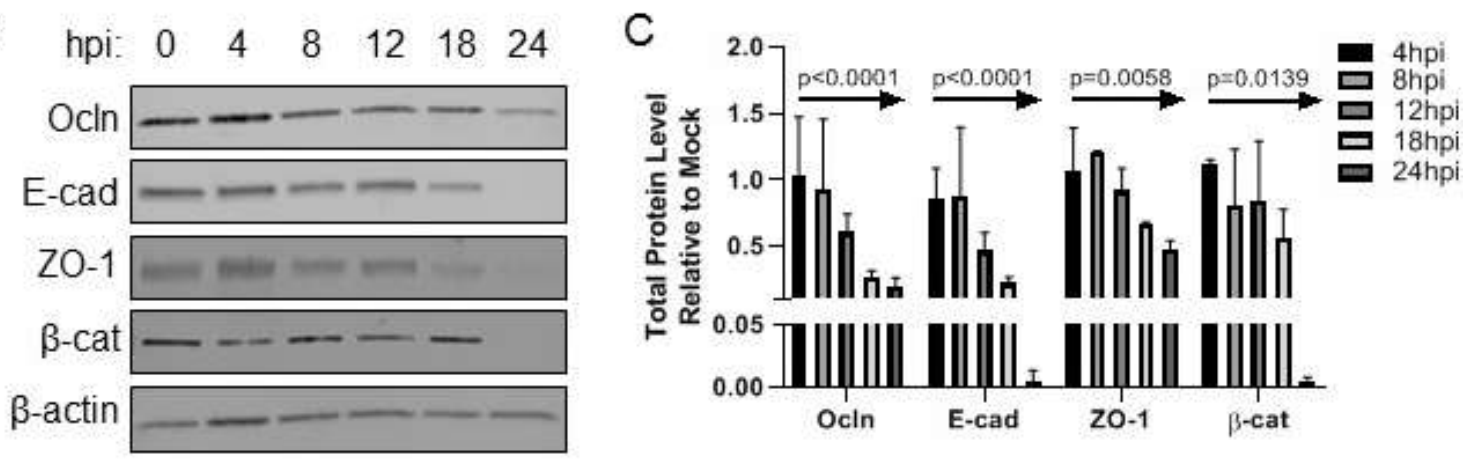

D
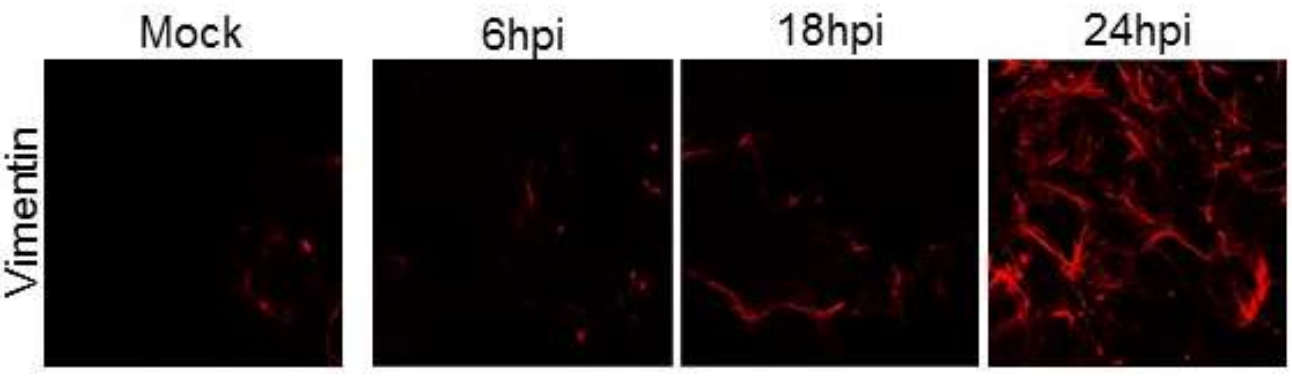


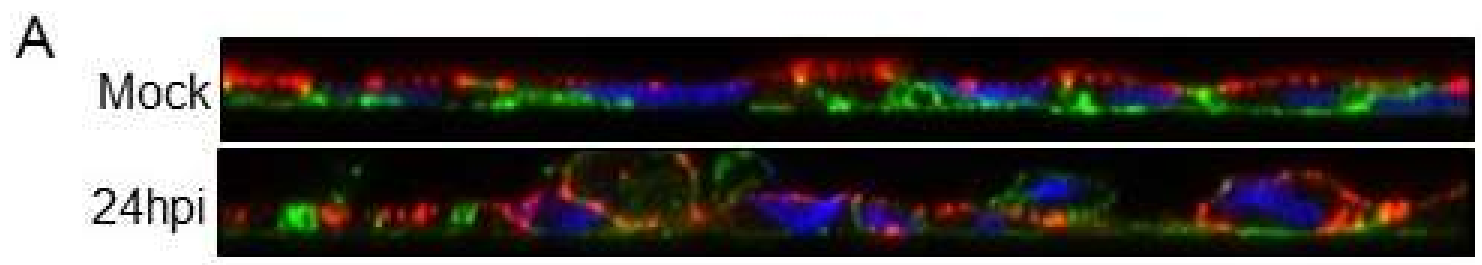

B
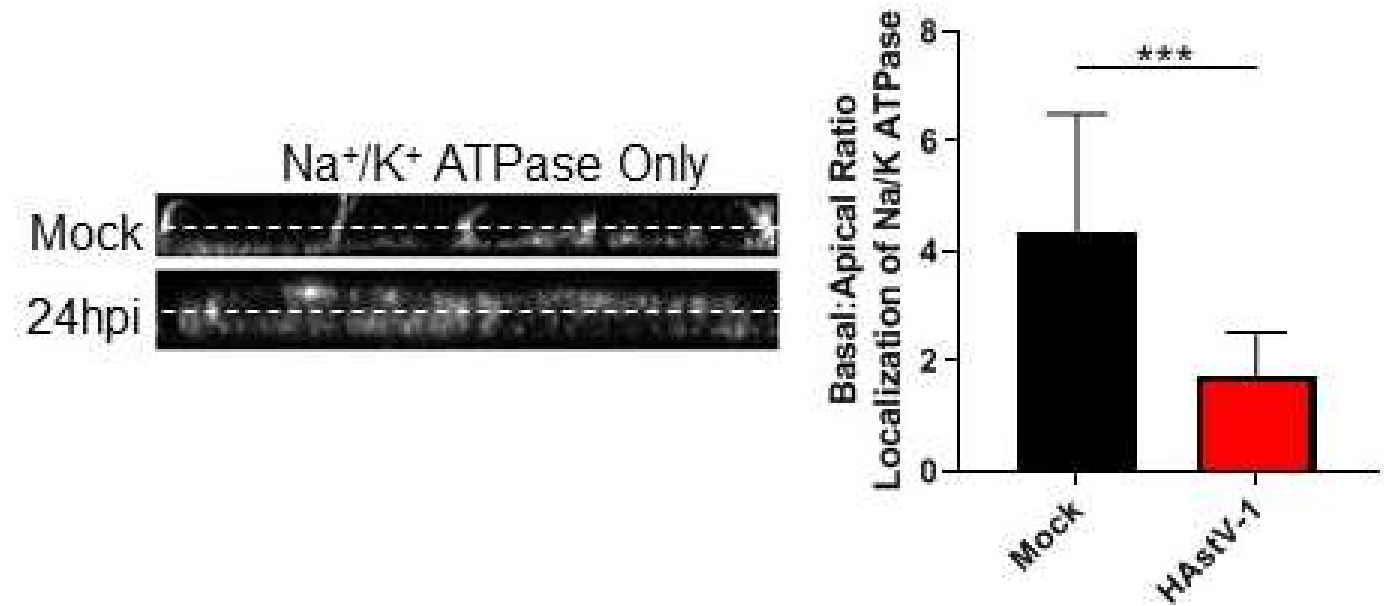

Figure 4-4. HAstV-1 Infection Leads to a Disruption of Cellular Polarity.

(A) Caco-2 infected with HAstV-1 or mock infected (as indicated). Cells were fixed at 24 hpi in 100\% ice-cold methanol and then stained for ezrin (red), sodium-potassium ATPase (green), and DAPI (blue). (B) Basal or apical localization of sodium-potassium ATPase was determined by measuring intensity above and below cell midline using ImageJ. Error bars indicate standard deviations, and asterisks show statistical significance as measured by the two-tailed student $\mathrm{t}$ test as follows: *, $\mathrm{P}<0.05 ; * *, \mathrm{P}<0.01$; ***, $\mathrm{P}<$ 0.001 . 


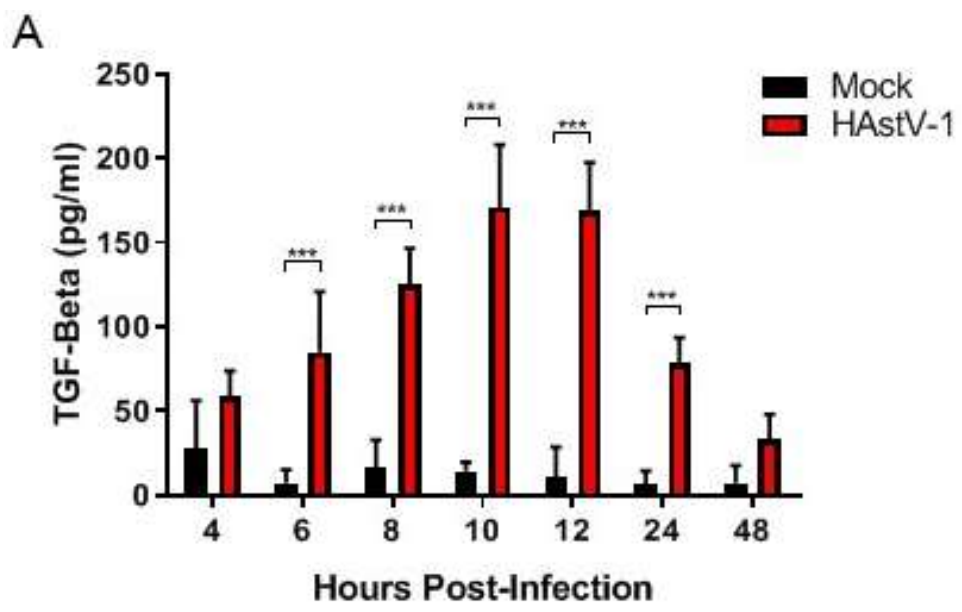

B

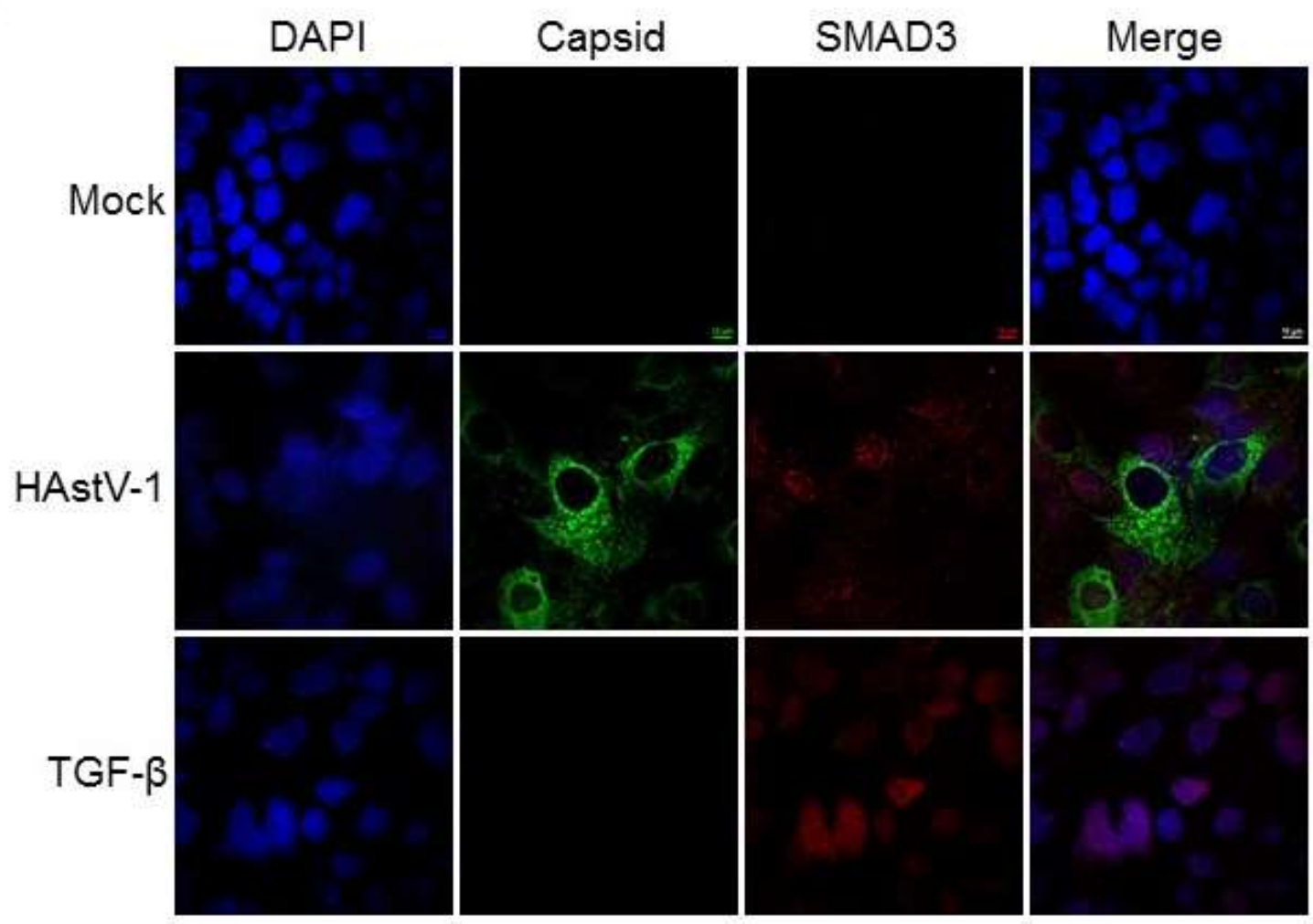

Figure 4-5. TGF- $\beta$ Activity Increases during the Course of HAstV-1 Infection. (A) Supernatants were collected from Caco-2 infected with HAstV-1 or mock infected from 4 to 48 hpi. Supernatants were then assayed for active TGF- $\beta$ using the PAI assay as described previously. ${ }^{344}$ Error bars indicate standard deviations, and asterisks show statistical significance as measured by two-way ANOVA followed by Sidak's multiple comparisons test as follows: *, $\mathrm{P}<0.05$; **, $\mathrm{P}<0.01$; ***, $\mathrm{P}<0.001$. (B) Caco-2 cells mock infected, HAstV-1 infected, or treated with $20 \mathrm{ng}$ of TGF- $\beta$ stained for SMAD3 (red), astrovirus capsid protein (green) and DAPI (blue). 
transducers for TGF- $\beta,{ }^{213}$ showed increased SMAD3 signal in uninfected bystander cells (Figure 4-5B). This suggests that uninfected cells in the monolayer may be responsible for the TGF- $\beta$ activation in response to HAstV infection.

\section{Inhibition of TGF- $\beta$ Activity Does Not Prevent HAstV-Induced EMT}

TGF- $\beta$ is considered the hallmark inducer of EMT. Thus, we asked if HAstVinduced EMT was dependent on TGF- $\beta$. To test, TGF- $\beta$ signaling was inhibited using the small molecule inhibitor $370,{ }^{369}$ which selectively inhibits the phosphorylation of the TGF- $\beta$ type I receptor. Despite inhibiting TGF- $\beta$ activity, SB431542 had no impact on HAstV-1-induced transcriptional regulation of genes associated with EMT

(Figure 4-6A), and failed to rescue E-cadherin protein expression (Figure 4-6B). Finally, the addition of active TGF- $\beta 1$ alone did not induce EMT with the same kinetics as HAstV did. We did not see loss of E-cadherin or disruption of polarity until 3 days postinoculation with $20 \mathrm{ng}$ TGF- $\beta$ (Figure 4-7). Overall, these studies suggest that activation of TGF- $\beta$ during infection is not the primary mechanism for HAstV-1-induced EMT. However, we cannot discount the possibility that TGF- $\beta$ plays a critical role in EMT induction in vivo or in low MOI infections.

\section{HAstV-Induced EMT Is Replication Dependent}

We previously demonstrated that HAstV-induced reorganization of occludin and increased barrier permeability were independent of viral replication both in vitro ${ }^{349}$ and in vivo. ${ }^{350}$ Thus, we asked if viral replication was necessary for EMT induction. To test, we inoculated Caco- 2 cells with UV-inactivated virus as previously described ${ }^{349}$ and assessed EMT. With the UV-inactivated virus failed to reduce E-cadherin expression or disruption of cellular polarity (Figure 4-8A, B). UV-activated virus also did not cause a decrease in $C D H 1$ or an increase in SNAII mRNA like observed with live virus (Figure 4-8C). Further, inhibition of ERK1/2 with U0126, which we demonstrated reduced HAstV replication, ${ }^{370}$ rescued some E-cadherin expression and cellular polarity (Figure 4-8D, E). These studies suggest that productive replication is required for the induction of EMT.

\section{Discussion}

We demonstrated the astroviruses increased barrier permeability by dysregulating tight junction, specifically occludin. ${ }^{349}$ These studies expand upon that data to demonstrate that HAstV induces epithelial cells to undergo EMT. This induction is characterized through transcriptional changes leading to a downregulation of CDH1 and OCLN early after infection followed by the upregulation of the key transcriptional factor SNAI1. This decrease of the transcription of key epithelial cell-specific genes is accompanied by the up regulation of mesenchymal cell-specific genes like VIM. These 
A

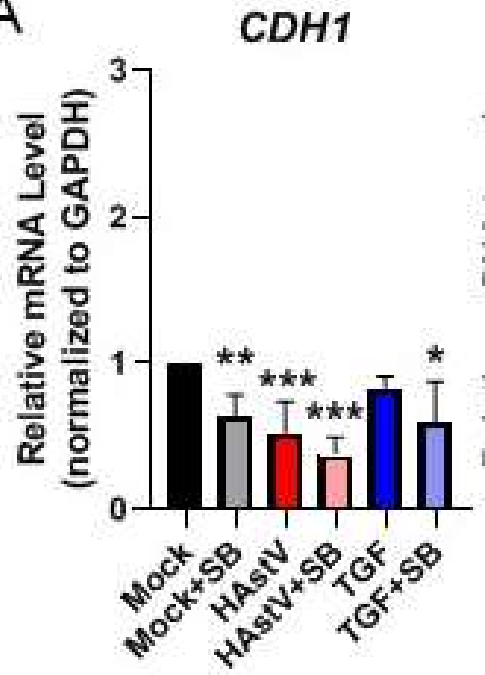

B

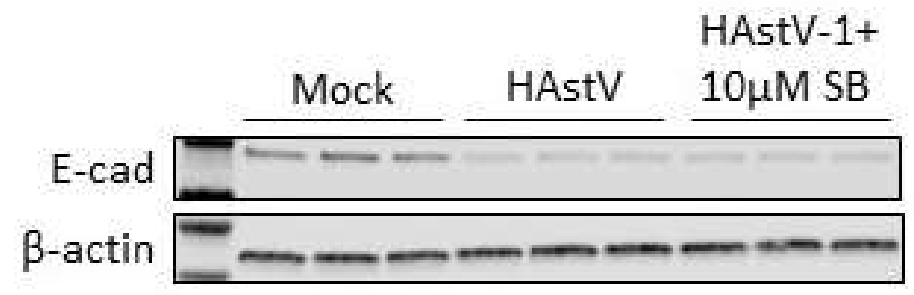

SNAI1
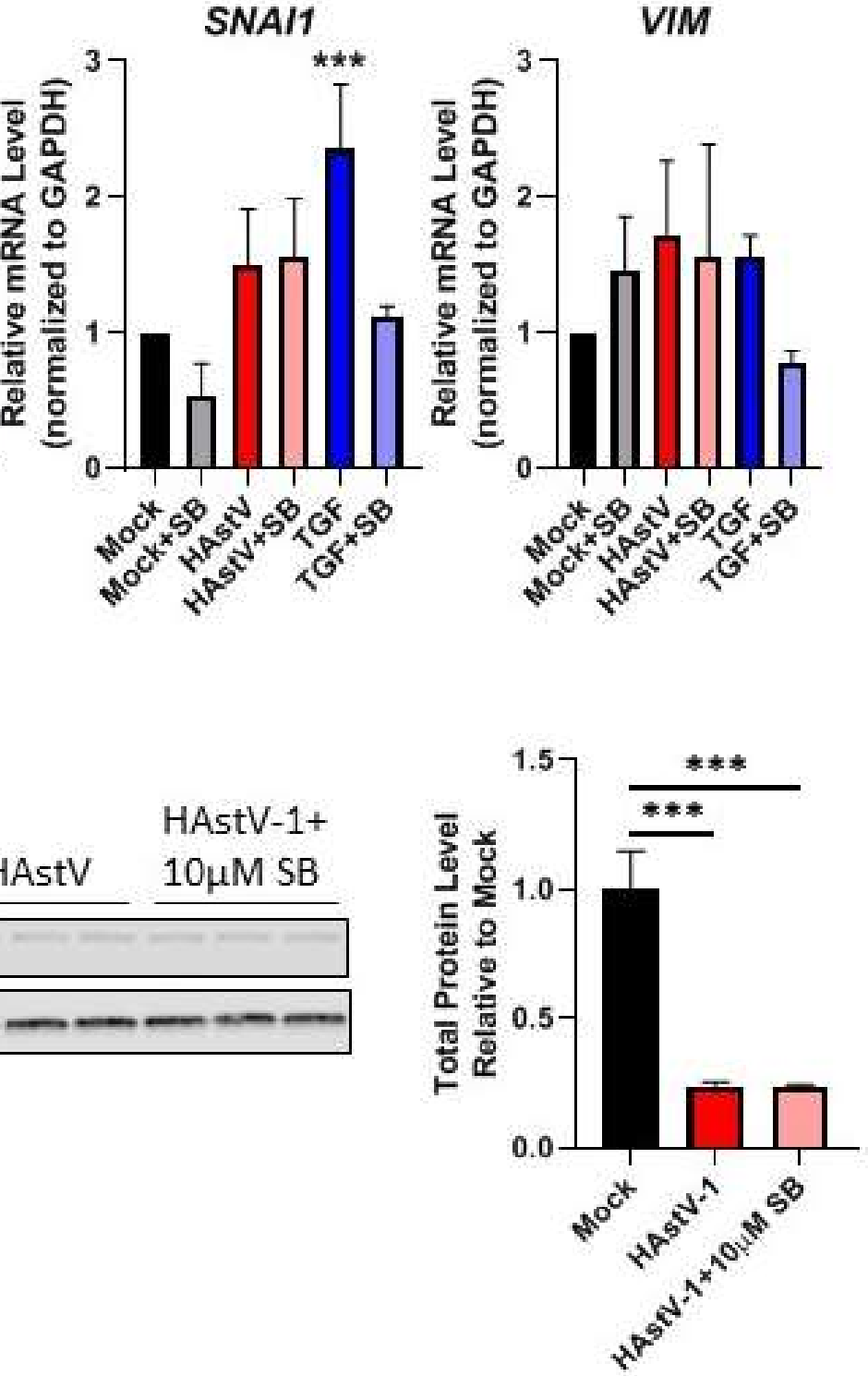

Figure 4-6. Inhibition of TGF- $\beta$ Signaling with SB431542 Does Not Inhibit HAstV-1-Induced EMT.

(A) RNA extracted at $24 \mathrm{hpi}$ from Caco-2 cells infected with HAstV-1, TGF- $\beta$ treated, or mock infected with or without $10 \mu \mathrm{M}$ SB431542 shows SB431542 does not rescue CDH1, SNAI1, or VIM regulation in HAstV-induced EMT. (B) Expression of epithelial marker, E-cadherin, was quantified by immunoblot of HAstV-1 or mock infected Caco-2 cell lysates with or without $10 \mu \mathrm{M} \mathrm{SB} 431542$. Bands were then quantified by densitometry and normalized to $\beta$-actin then compared to mock-infection. All error bars indicate standard deviations, and asterisks show statistical significance as measured by ordinary one-way ANOVA followed by Dunnett's multiple comparisons test as follows: *, $\mathrm{P}<0.05 ; * *, \mathrm{P}<0.01 ; * * *, \mathrm{P}<0.001$. 


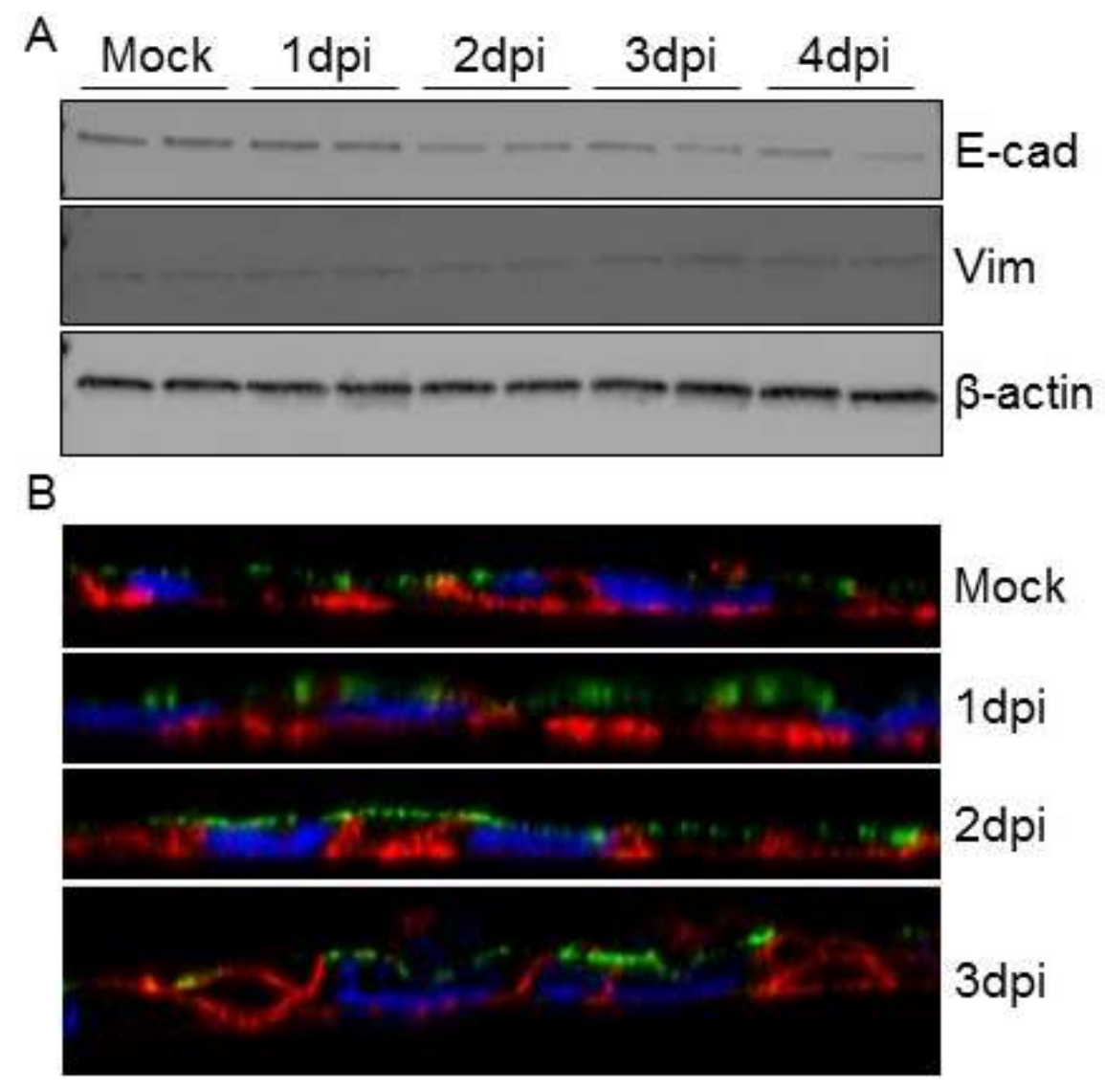

Figure 4-7. TGF- $\beta$-Induced EMT in Caco-2 Cells.

(A) Western blot of E-cadherin and vimentin at 1,2,3, and 4 days post inoculation with 20 ng active TGF- $\beta$ compared to mock. (B) Caco-2 inoculated with $20 \mathrm{ng}$ active TGF- $\beta$ or mock treated (as indicated). Cells were fixed at 1, 2, or 3 days post-inoclation in 100\% ice-cold methanol and then stained for ezrin (green), sodium-potassium ATPase (red), and DAPI (blue). 
Figure 4-8. Replication Is Required for HAstV-1-Induced EMT.

(A) Expression of epithelial marker, E-cadherin, was quantified by immunoblot of HAstV-1, UV-inactivated HAstV-1, or mock infected Caco-2 cell lysates. Bands were then quantified by densitometry and normalized to $\beta$-actin then compared to mockinfection. (B) RNA extracted at 24 hpi from Caco-2 cells infected with HAstV-1, UVinactivated HAstV-1, or mock infected show UV-inactivated virus does not modulate CDH1 or SNAI1 regulation as active HAstV-1 does. (C) Sodium potassium ATPase localization in Caco-2 cells infected with UV-inactivated HAstV-1 shows no difference to mock infected cells. (D) Expression of E-cadherin was quantified by immunoblot of HAstV-1, HAstV-1 in the presence of $10 \mu \mathrm{M}$ U0126, or mock infected Caco-2 cell lysates. Bands were then quantified by densitometry and normalized to $\beta$-actin then compared to mock-infection. (E) Sodium potassium ATPase localization in Caco-2 cells infected with HAstV-1 in the presence of $10 \mu \mathrm{M}$ U0126 and mock infected cells. All error bars indicate standard deviations, and asterisks show statistical significance as measured by ordinary one-way ANOVA followed by Dunnett's multiple comparisons test as follows: *, P $<0.05 ; * *, \mathrm{P}<0.01 ; * * *, \mathrm{P}<0.001$. 
A

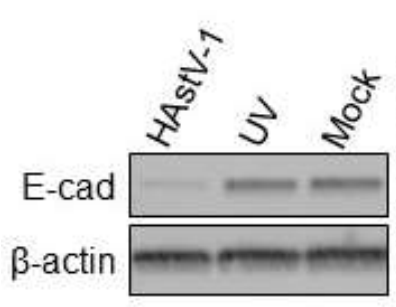

C

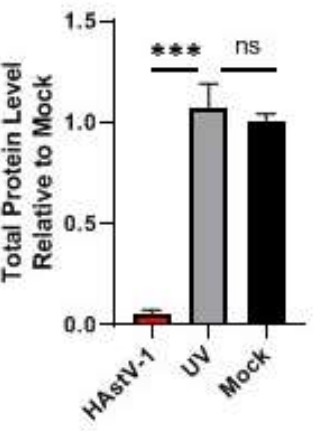

B
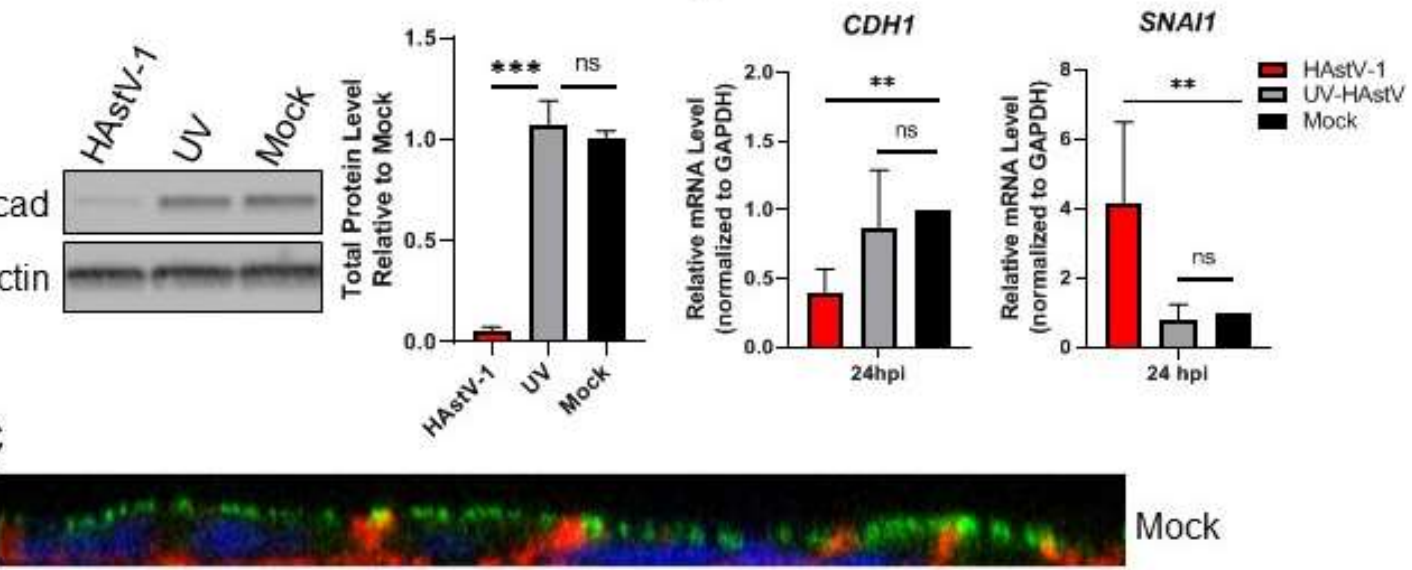

Mock

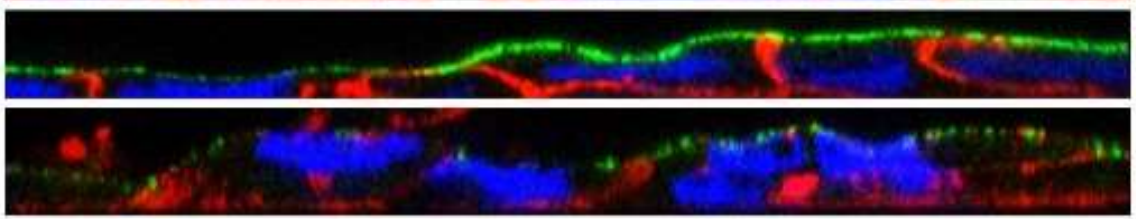

UV

HAstV-1

D
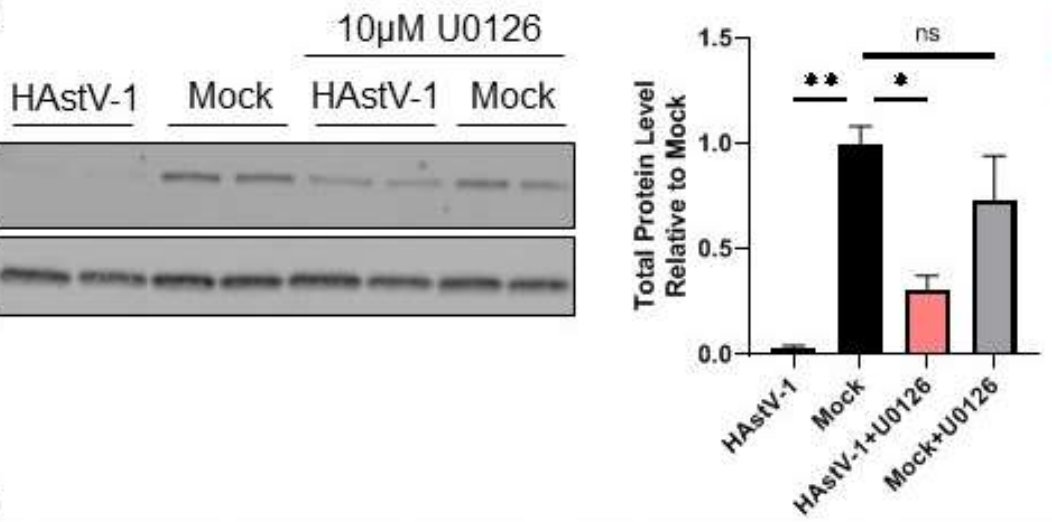

E

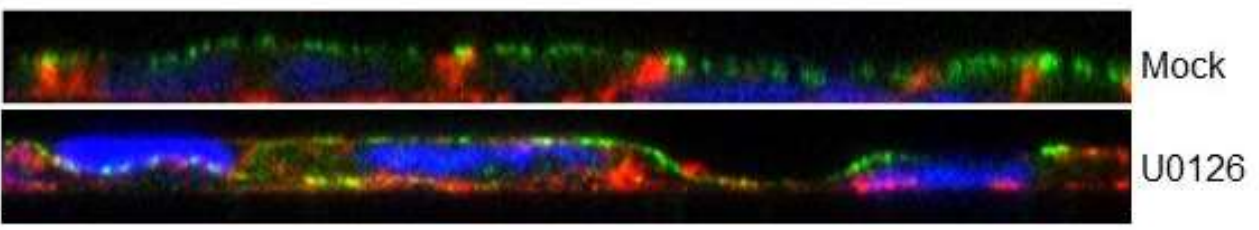


transcriptional changes result in decreased epithelial protein levels leading to a breakdown of the cell junctions resulting from the loss of those proteins from the cell periphery. Accompanying this is a subsequent loss of cellular polarity by 24 hpi. Despite an increase in active TGF- $\beta$ during HAstV infection, inhibition of TGF- $\beta$ signaling did not prevent the EMT phenotype; HAstV-induced EMT is replication dependent. EMT is a rare phenomenon triggered by only a few viruses and even less non-oncogenic viruses, making $\mathrm{HAstV}$-induced EMT a truly unique finding.

The observation that HAstV induces EMT during infection generates more questions that will need to be resolved before we will fully understand its role in HAstV pathogenesis. The first question raised by our findings concerns the benefit of inducing EMT during infection. For the oncogenic viruses that induce EMT it's simple; this process promotes metastasis, enabling these viruses to spread throughout the host, clearly benefiting the virus. However, for the non-oncogenic viruses it's not as clear.

Researchers studying EMT in rhinovirus and TGEV infection propose similar rationale as to why their virus of interest induces EMT. Faris et al. suggest rhinovirus infection alters the structure and function of airway epithelium and increases the susceptibility to secondary infection. By facilitating secondary infection, rhinovirus can alter the cytokine and chemokine responses. ${ }^{361} \mathrm{Xia}$ et al. also suggest TGEV induces EMT to promote colonization of ETEC in the intestinal epithelium. ${ }^{341}$ Alone TGEV provokes a robust cytokine response, however upon co-infection cytokine levels were decreased, possibly assisting to decrease clearance of both pathogens. ${ }^{371,372}$ At first glance, it would appear that inducing EMT would be beneficial for HAstV. This process produces gaps in the intestinal barrier that the virus can exploit and gain access to the rest of the body. We have seen in the turkey model and now in cases of HAstV infection that astrovirus has the potential for systemic spread. However, there could be an argument for this process to benefit the host. In our polarity staining, we noticed cells that appeared to be extruded out of the cell monolayer (Figure 4-4A). We questioned if the host was inducing EMT in a wound healing response, where infected epithelial cells are extruded and mesenchymal cells fill in the gaps in the monolayer. This could prevent extensive damage and inflammation for the host which is a unique factor already seen in astrovirus infections. ${ }^{39,40}$

The extrusion of infected cells could still prove to be a benefit to the virus, since infected cells would be released into the intestinal lumen, shed in feces and allow for spread to new hosts. This raises another questioning the link between EMT and symptoms of astrovirus infection. Previous research has attempted to link the barrier permeability seen during infection with the main symptom of astrovirus, diarrhea. Recent reports now show an association with astrovirus infection and severe extragastrointestinal symptoms, ${ }^{152}$ such as encephalitis and meningitis. We know that HAstV is able to disrupt barrier integrity via EMT, but it is unknown whether this is specific to intestinal barriers or if could extend to others such as the blood brain barrier. Investigations into the association of symptoms and EMT are currently ongoing. Determining if EMT plays a role in the dissemination of astrovirus from the gastrointestinal tract to the central nervous system could even provide new therapeutic targets for systemic HAstV infection. 
Previously we have shown that barrier permeability and disruption of some junctional proteins such as occludin is replication independent. ${ }^{349}$ Yet, here we show that replication is required for the induction of EMT. We hypothesize that there may be a binding event that is sufficient to cause some barrier permeability and the re-localization of occludin thus allowing the capsid protein alone to cause these events. However, a more intricate signaling cascade is triggered during replication of the virus that induces the EMT phenotype. Further research is needed to determine the exact aspect of replication that is triggering EMT, such as the processing of a non-coding part of the genome or the production of a non-structural protein.

Although the cleavage process of the HAstV nonstructural polyproteins is still not well characterized, it has been suggested at least four nonstructural proteins in addition to an RNA-dependent RNA polymerase ( $\mathrm{RdRp})$ are produced during replication. ${ }^{373}$ However, the exact function of these nonstructural proteins remains undetermined. It has been shown that genetic variation in at least one of the HAstV nonstructural proteins can impact viral RNA production and the amount of infectious virus shed. ${ }^{374,375}$ Studies of the oncogenic viruses that induce EMT have shown strong evidence that viral proteins are heavily involved. The $\mathrm{X}$ protein of $\mathrm{HBV},{ }^{354,376-378} \mathrm{NS}^{3} \mathrm{~B}^{379}$ and core protein of $\mathrm{HCV},{ }^{380-}$ ${ }^{382} \mathrm{E}^{383}$ and E6/E7 oncoproteins of $\mathrm{HPV},{ }^{384,385}$ nuclear antigens ${ }^{386}$ and latent membrane proteins of EBV, ${ }^{387-389}$ and immediate early proteins 1 and 2 of $\mathrm{CMV}^{358}$ have all been shown to induce EMT. While viral proteins have not been reported to play a role in EMT for rhinovirus or TGEV, studies on EMT with these two viruses are limited and therefore should not be ruled out. Given this, it could be possible one of the nonstructural proteins or a cleavage product may impact the induction of EMT during HAstV infection. While we currently lack the tools necessary for additional studies focused on the nonstructural proteins, we are in the process of developing them. These studies will be critical in order to definitively identify the viral factors involved in HAstV-induced EMT.

During replication, there is also may be the processing of microRNAs (miRNA). Recently there have been many reports in the literature of non-coding miRNAs regulating EMT. ${ }^{270,271}$ Many have been shown to inhibit EMT, like the miR-220 family members (miR-200a, miR-200b, miR-200c, miR-141, and miR-429) that directly repress ZEB1/2. ${ }^{273-276}$ In addition, miR-30a inhibits EMT non-small cell lung cancer by directly targeting Snail. ${ }^{277}$ However, not all miRNAs inhibit EMT, overexpression of miR-10b initiates metastasis by inhibiting translation of HOXD10, resulting in increased expression of RhoC. ${ }^{390}$ Furthermore, miR-373 and miR-520c stimulate tumor invasion by down-regulating CD44. ${ }^{284}$ At this time, there are more than seventy different miRNAs reported to be involved in EMT regulation. ${ }^{269}$ It is known that many viruses encode their own miRNAs, ${ }^{391}$ and in the case of EBV these miRNAs have been reported to facilitate EMT. ${ }^{392,393}$ Ongoing studies in our lab is looking at cellular miRNAs modulated during HAstV infection as well as identifying miRNAs encoded within the astrovirus genome. Since we know HAstV-induced EMT is replication dependent, it would make sense that something during replication like the processing of a miRNA could be inducing EMT. 
While TGF- $\beta$ is induced during HAstV replication, here we show the TGF- $\beta$ inhibitor, SB431524 did not inhibit HAstV-induced EMT. SB431542 acts by inhibiting the phosphorylation of the type I TGF- $\beta$ receptor, and preventing the activation of SMADs. ${ }^{369}$ However, TGF- $\beta$ can activate EMT through SMAD independent pathways, that would not be affected by SB431542. ${ }^{369}$ For example, TGF- $\beta$ can induce the activation of the PI3K/Akt pathway independent of SMADs. This pathway has also been previously reported to be active during HAstV infection. ${ }^{394}$ However, if this pathway was responsible for induction of EMT in our system, we would expect to see a either an upregulation of Twist or a down regulation of GSK-3 $\beta,{ }^{395}$ neither of which is indicated by our data (Figure 4-2). PI3K/Akt can also crosstalk with Wnt/ $\beta$-catenin signaling. However, this signaling would result in $\beta$-catenin accumulating in the nucleus, which is not seen in Figure 4-1. Although our data suggests that TGF- $\beta$ is likely not the main mechanism utilized by HAstV to induce EMT, we believe it is still an important factor in astrovirus pathogenesis. TGF- $\beta$ is upregulated in activity both in vitro and in vivo, ${ }^{39}$ so it must be an integral part of the viral replication cycle. Interestingly, our SMAD staining showed that TGF- $\beta$ signaling is mostly occurring in non-infected cells in the epithelial monolayer. This bystander effect is likely very important in vivo where TGF- $\beta$ can create an immune suppressive microenvironment ${ }^{396}$ and could account for the lack of inflammation see in in vivo astrovirus infections. Future studies will look at the role of TGF- $\beta$ activation in astrovirus replication.

In conclusion, we demonstrated HAstV induces EMT in a replication dependent manner. This finding not only leads us one step closer in understanding astrovirus pathogenesis but also in understanding EMT from the perspective of a non-oncogenic virus. EMT leads to dysfunction of the epithelial barrier. In vivo, barrier dysfunction in the gastrointestinal system results in gaps in the epithelium that pathogens can take advantage of to spread throughout the body. Previous research has already shown the capability of astrovirus to spread systemically in turkey poults. Recent reports are now showing more cases of systemic HAstV infection, even causing severe CNS symptoms like encephalitis and meningitis. During systemic infections, the immune system becomes overwhelmed and cannot clear the virus alone. In addition, the majority of severe HAstV infections occur in individuals that already have compromised immune systems. Currently, there are no antiviral treatment options for HAstV infection. When these cases arise, clinicians and physicians do not have proven treatments to offer patients. In the next chapter, we sought to determine if any known antivirals were effective in treating astrovirus infection. 


\section{CHAPTER 5. ASTROVIRUS REPLICATION IS INHIBITED BY NITAZOXANIDE IN VITRO AND IN VIVO ${ }^{2}$}

\section{Introduction}

Diarrheal disease is the second leading cause of death in children under 5 years of age, with nearly 1.7 billion cases and 525,000 deaths each year ${ }^{397}$ Since their discovery in 1975, human astroviruses (HAstV), which are positive single-stranded RNA viruses, have consistently ranked among the leading causes of diarrhea worldwide. ${ }^{127}$ However, human astrovirus infections can range from asymptomatic to mild diarrhea or rare instances of fatal systemic disease. ${ }^{76,84}$ Infections in immunocompetent individuals typically present as watery diarrhea that resolves within 1 to 3 days post-infection without the need for hospitalization. ${ }^{127}$ Astrovirus outbreaks occur frequently in assisted living facilities, hospitals, and child care centers, where the young, elderly, and immunocompromised populations are at risk of persistent diarrhea, leading to wasting, ${ }^{155}$ and extragastrointestinal disease that may require medical intervention, including respiratory disease, ${ }^{398-401}$ fatal encephalitis, and meningitis. ${ }^{87}$

Despite its high prevalence and the risk of severe disease, no vaccines or drug treatments exist for astrovirus. Only oral or parenteral fluids and electrolytes are available to prevent and treat dehydration caused by astrovirus-induced diarrhea. In these studies, we provide the first evidence that nitazoxanide (NTZ), an FDA-approved broad-spectrum antiparasitic and antiviral drug, inhibits the replication of multiple strains of human astrovirus in vitro even when administered up to $8 \mathrm{~h}$ post-infection and reduces viral shedding and diarrhea in vivo. This work highlights the potential use of NTZ as an effective therapeutic strategy against astrovirus infection.

\section{Results}

\section{Nitazoxanide (NTZ) Blocks Astrovirus Replication In Vitro}

To identify an effective antiviral drug against astrovirus, human colon carcinoma (Caco-2) cells were infected with a laboratory strain of human astrovirus strain 1 (HAstV-1) at a multiplicity of infection (MOI) of 1 before increasing concentrations of antiviral compounds or dimethyl sulfoxide (DMSO; vehicle control) were added $1 \mathrm{~h}$ postinfection (hpi). The panel of antiviral compounds used in our study, foscarnet, ribavirin, acyclovir, and nitazoxanide, were chosen upon the recommendation of clinical colleagues, with acyclovir serving as a negative control. Viral capsid protein levels were quantitated at 24 hpi by immunofluorescence microscopy as described previously. ${ }^{343}$

\footnotetext{
${ }^{2}$ Modified from final submission with permission. Hargest V, Sharp B, Livingston B, Cortez V, SchultzCherry S. Astrovirus Replication Is Inhibited by Nitazoxanide In Vitro and In Vivo. J. Virol. 94, (2020) doi: $10.1128 /$ JVI.01706-19. ${ }^{342}$
} 
Foscarnet, ribavirin, and acyclovir failed to inhibit HAstV-1 replication even at concentrations of $250 \mu \mathrm{M}$ (Figure 5-1A). In contrast, NTZ inhibited HAstV-1 replication in a dose-dependent manner, with the $5 \mu \mathrm{M}$ treatment completely blocking virus replication (Figure 5-1A, B). The 50\% effective concentration $\left(\mathrm{EC}_{50}\right)$ was calculated as approximately $1.47 \mu \mathrm{M}$ (Figure 5-1C). Concentrations of NTZ above $5 \mu \mathrm{M}$ were associated with decreased cell viability compared to the cell viability with vehicle alone (DMSO) (Figure 5-2). Thus, subsequent studies were performed with NTZ at a concentration of $2.5 \mu \mathrm{M}$.

\section{NTZ Inhibits dsRNA Production}

To determine the stage of the viral replication cycle blocked by NTZ, drug was added at 2, 4, 6, 8, and $12 \mathrm{~h}$ after HAstV-1 infection and viral capsid expression quantitated (Figure 5-3A). The addition of NTZ up to 8 hpi completely inhibited HAstV1 replication (Figure 5-3B, D), suggesting it blocks a stage of the viral life cycle following entry and uncoating but before structural protein production. Indeed, NTZ reduced the formation of double-stranded RNA (dsRNA) that occurs when the astrovirus genome is generated via its RNA-dependent RNA polymerase (Figure 5-3C, E). This method serves as a proxy for replication, given our lack of antibodies to the HAstV-1 nonstructural proteins.

Previous work demonstrated that thiazolide, the active form of NTZ, upregulates type I and II interferon (IFN) genes, which were critical for reducing HIV replication in human peripheral blood mononuclear cells (PBMCs), ${ }^{402}$ Type I IFN limits HAstV replication. ${ }^{58}$ Thus, to determine whether NTZ increased type I or III IFN within the time frame it inhibits HAstV replication, we treated Caco-2 cells with NTZ or DMSO (untreated) and quantitated interferon alpha (IFN- $\alpha$ ), IFN- $\beta$, or IFN- $\lambda$ mRNA levels at 2 and $4 \mathrm{~h}$. At $4 \mathrm{~h}$ post-NTZ treatment, the shortest duration of time NTZ can be added and still inhibit HAstV dsRNA production, IFN- $\alpha$, IFN- $\beta$, and IFN- $\lambda$ mRNA transcripts were not significantly upregulated compared to their levels in DMSO-treated cells

(Figure 5-4), suggesting that NTZ inhibits HAstV independent of IFN induction. Future studies will explore the mechanism of action.

\section{NTZ Is Effective Across HAstV Serotypes}

We next asked if NTZ was effective against multiple classical HAstV serotypes and clinical isolates. Briefly, Caco-2 cells were infected with four different laboratoryadapted HAstV serotypes (HAstV-1, -2, -6, and -8) and four clinical isolates obtained from patient samples (SJ177.110 [HAstV-2], SJ60.212 [HAstV-8], SJ88123.E120 [HAstV-1], and SJ88027.E259 [HAstV-1]) and treated with $2.5 \mu \mathrm{M}$ NTZ or DMSO (vehicle control). NTZ completely inhibited all HAstV isolates, suggesting it is broadly protective against multiple HAstV serotypes (Figure 5-5). 

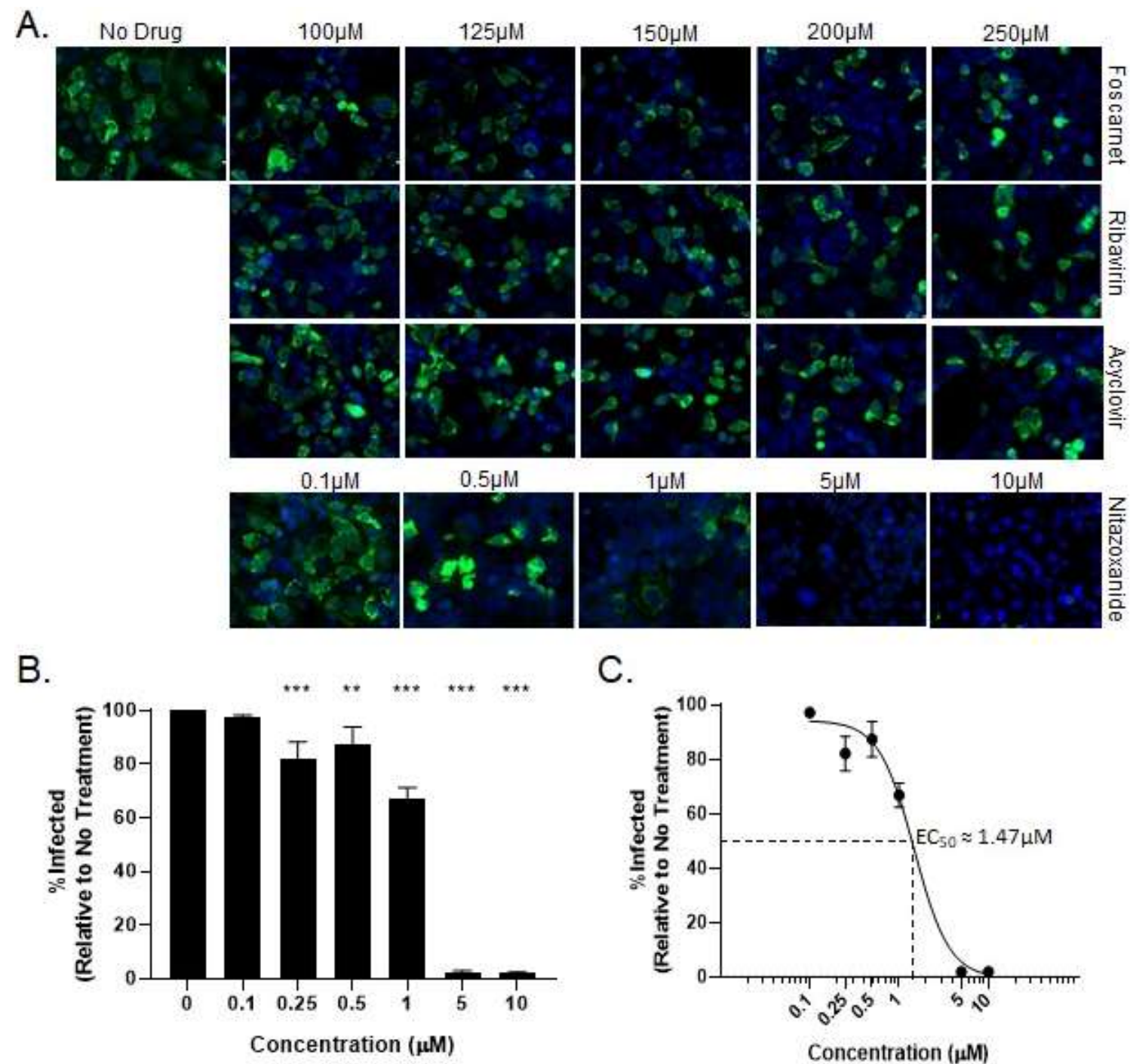

C.

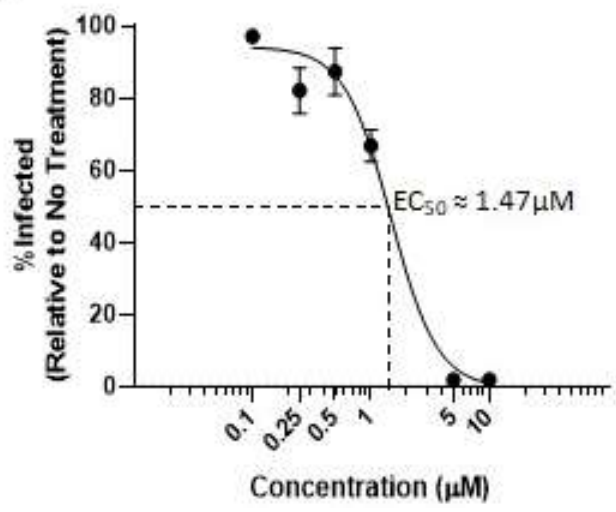

Figure 5-1. Nitazoxanide (NTZ) Inhibits HAstV-1 Replication in Caco-2 Cells. (A) Caco-2 cells were infected with HAstV-1 at an MOI of 1 and treated with a panel of antivirals (foscarnet, ribavirin, acyclovir, and nitazoxanide) at the indicated concentrations. At $24 \mathrm{hpi}$, cells were fixed and stained with DAPI (blue) and for the presence of astrovirus capsid protein (green). (B) The percentages of infected cells were calculated and compared to the results for nontreated cells. (C) Nonlinear regression analysis of percent infection data was used to determine the $50 \%$ effective concentration (EC50). All error bars indicate standard errors of the means, and asterisks show statistical significance as measured by ordinary one-way ANOVA as follows: *, P 0.05; **, P 0.01; ***, P 0.001 . 


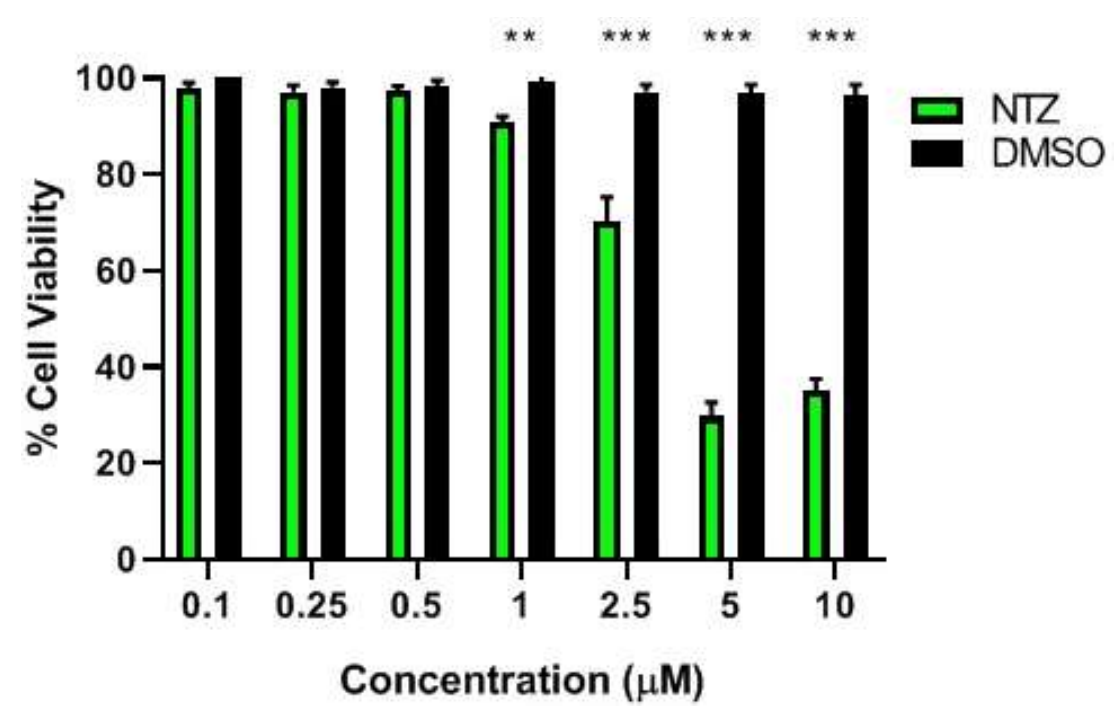

Figure 5-2. Cytotoxicity of NTZ in Caco-2 Cells.

Cell viability of Caco-2 cells following $24 \mathrm{~h}$ of treatment with NTZ or vehicle alone (DMSO) at the indicated concentrations was determined by MTT assay. All error bars indicate standard errors of the means, and asterisks show statistical significance as measured by multiple $t$ tests as follows: *, P 0.05 ; **, P 0.01 ; **, P 0.001 . 
Figure 5-3. Nitazoxanide Inhibits HAstV-1 Replication In Vitro When Added Up to 8 hpi.

(A) Caco-2 cells were infected with HAstV-1, and at the indicated times post-infection, 2.5M NTZ or vehicle alone (DMSO) was added. (B) At 24 hpi, cells were fixed and stained with DAPI (blue) and for the presence of astrovirus capsid protein (green). (C) At 10 hpi, cells were fixed and stained with DAPI (blue) and for the presence of dsRNA (green). (D) Quantification of the percentages of cells with capsid staining from the experiment whose results are shown in panel B. (E) Quantification of the percentages of cells with dsRNA staining from the experiment whose results are shown in panel C. All error bars indicate standard errors of the means, and asterisks show statistical significance as measured by multiple $t$ tests as follows: *, P 0.05 ; **, P 0.01 ; ***, P 0.001 . 


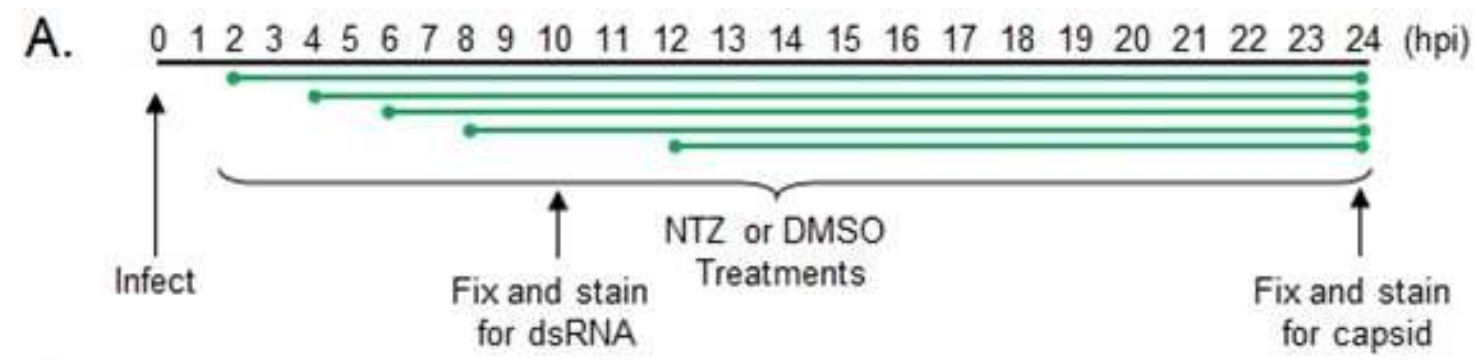

B.

$2 \mathrm{hr}$

$4 \mathrm{hr}$

$6 \mathrm{hr}$

$8 \mathrm{hr}$

$12 \mathrm{hr}$

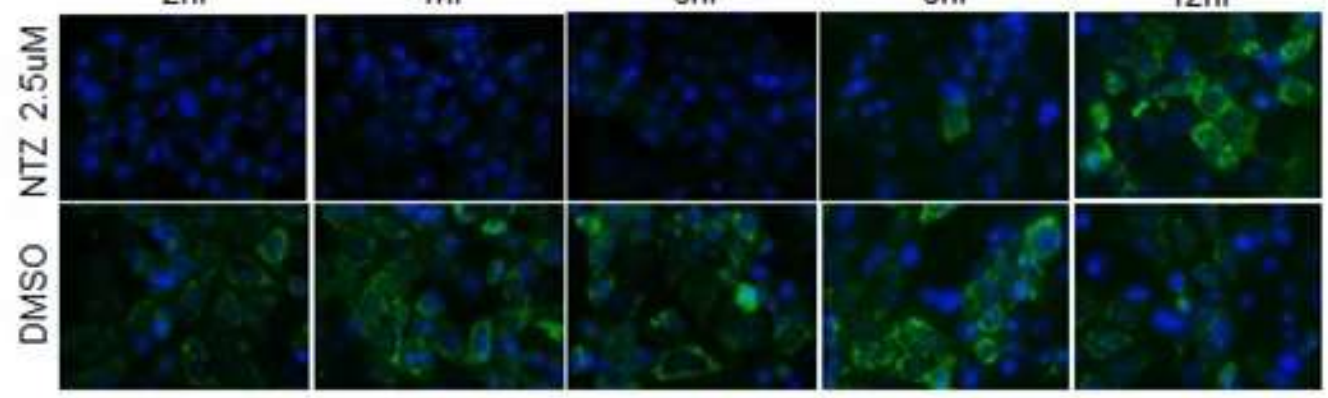

C.
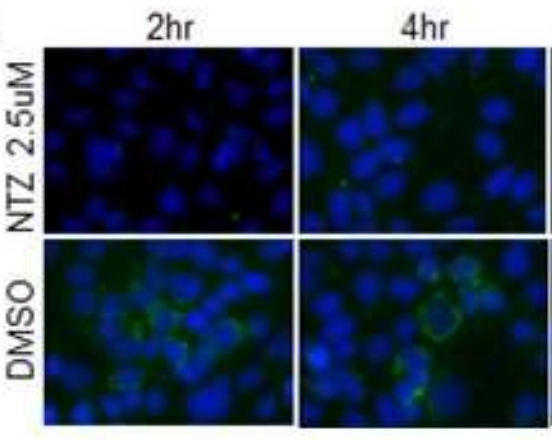

$6 \mathrm{hr}$

$8 \mathrm{hr}$

D.

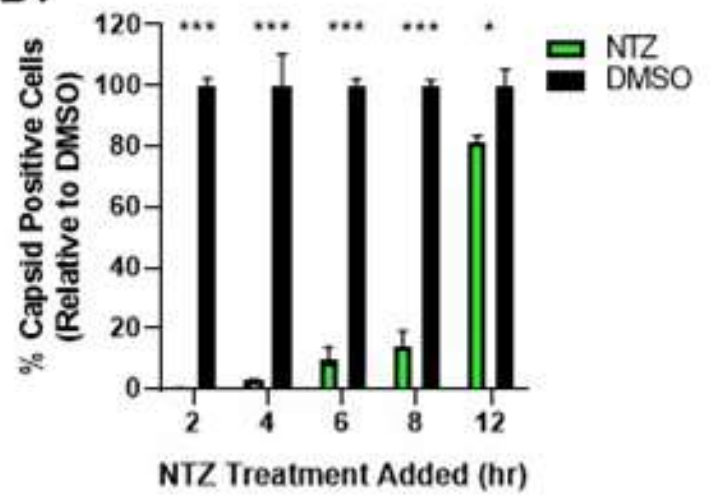

E.

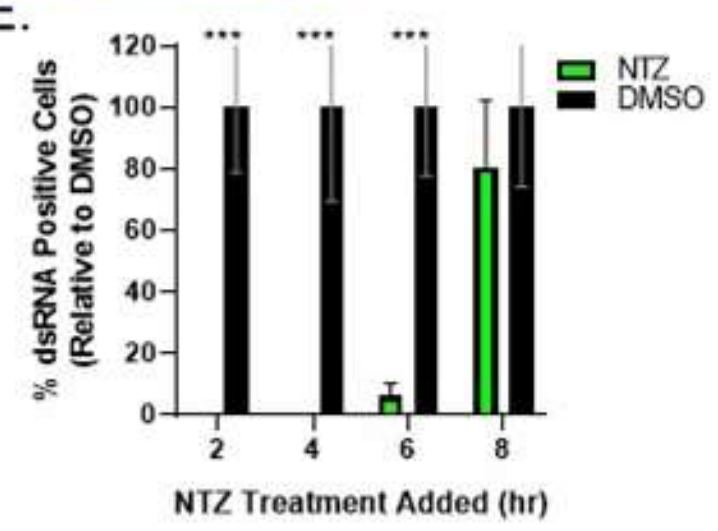




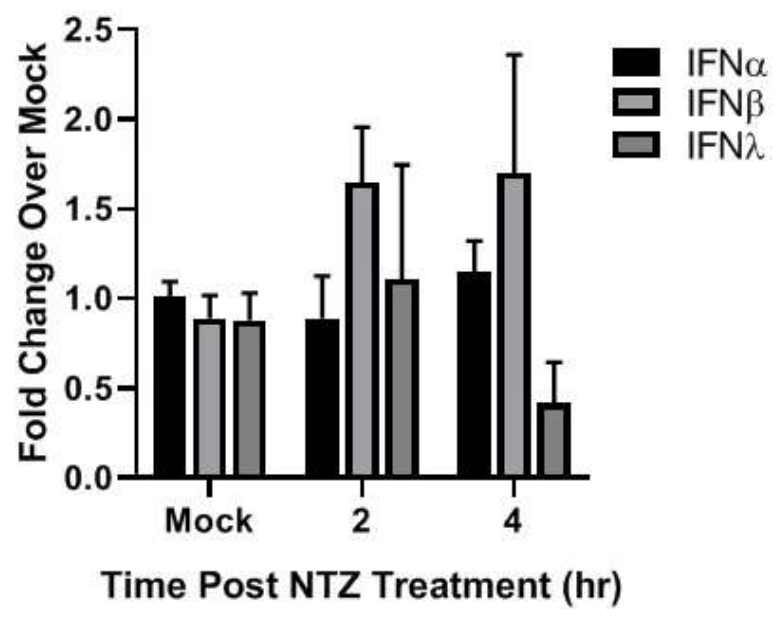

Figure 5-4. NTZ Does Not Induce Significant IFN Levels in Caco-2 Cells.

Real-time RT-PCR for IFN- $\alpha$, IFN- $\beta$, and IFN- $\lambda$ was performed on RNA collected from Caco-2 cells treated with $2.5 \mathrm{M} \mathrm{NTZ}$ and normalized to the value for GAPDH. Results are shown as fold increases over the results for untreated cells, and error bars indicate standard errors of the means. 


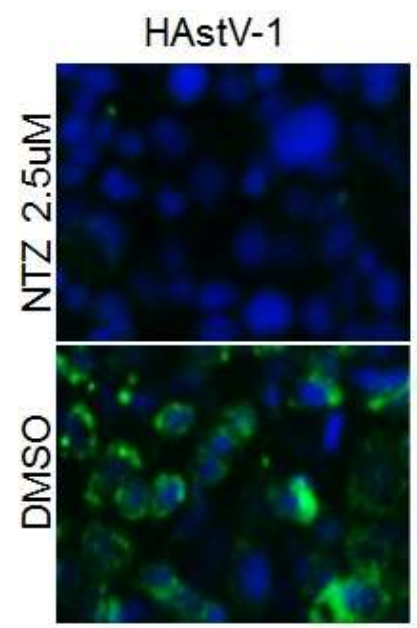

HAstV-2

HAstV-6

HAstV-8
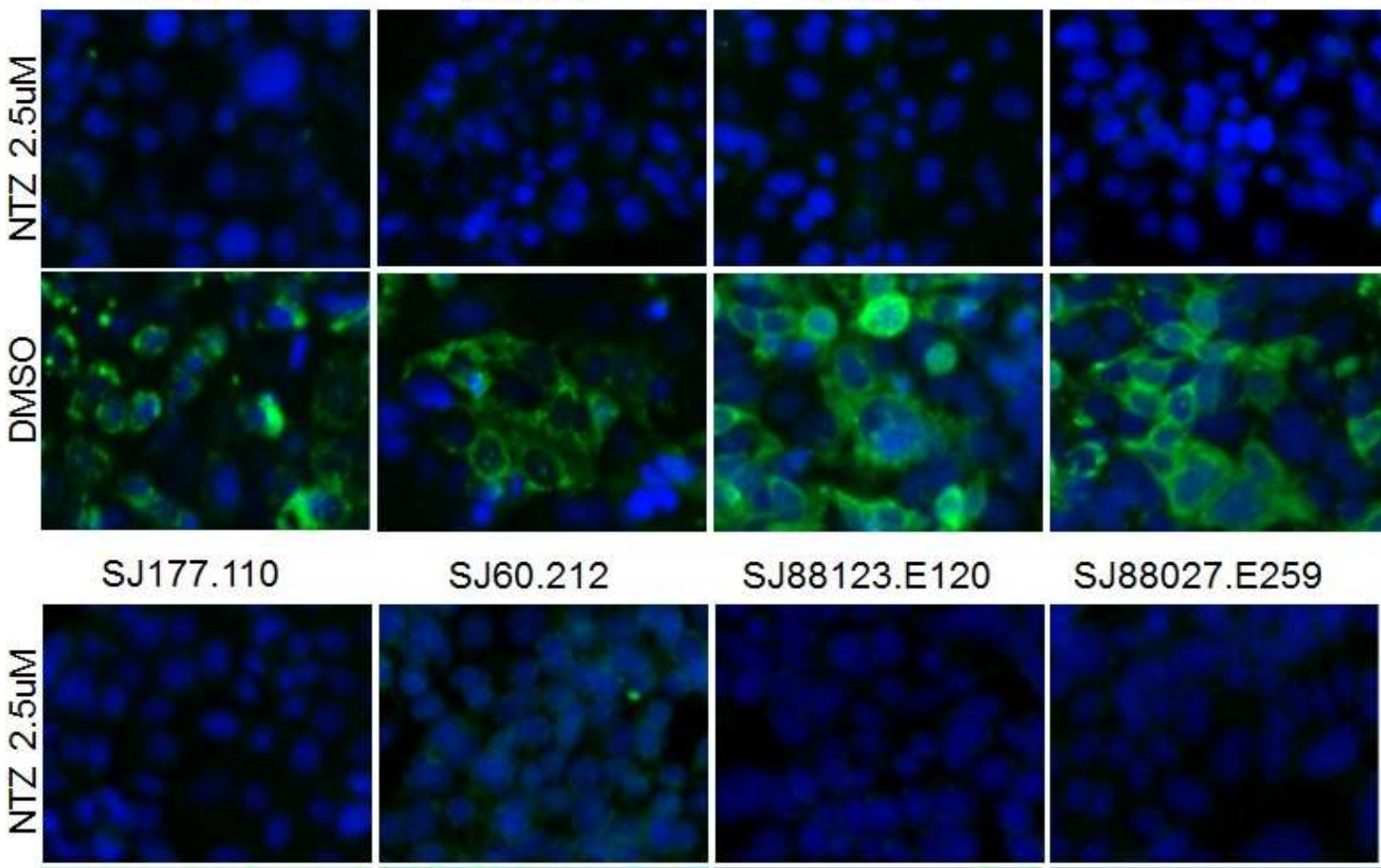

SJ88123.E120

SJ88027.E259

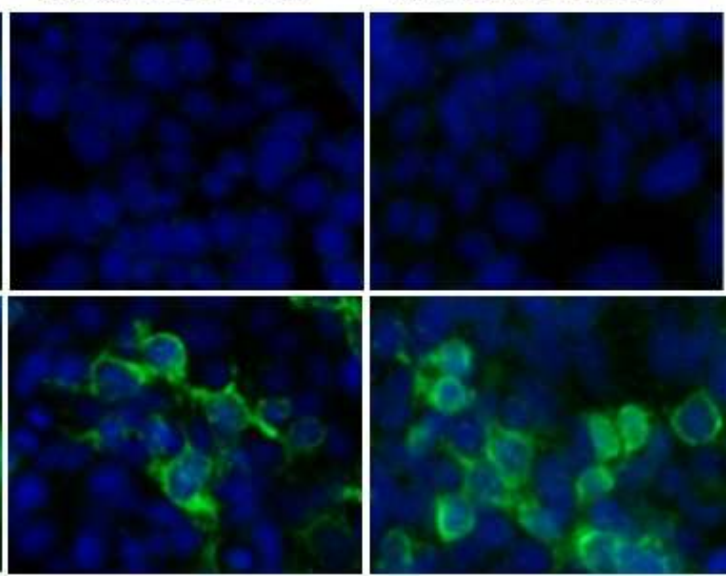

Figure 5-5. Nitazoxanide Inhibits the Replication of Multiple Serotypes and Clinical Isolates of Human Astrovirus.

Caco-2 cells were infected with laboratory-adapted virus serotypes (top) or clinical isolates (bottom) and treated with 2.5 M NTZ or vehicle alone (DMSO). At 24 hpi, cells were fixed and stained with DAPI (blue) and for the presence of astrovirus capsid protein (green). 


\section{NTZ Reduces Viral Replication and Clinical Disease In Vivo}

To determine if NTZ reduced disease in vivo, we used the only small animal model exhibiting astrovirus-induced diarrheal disease, turkey poults. ${ }^{39}$ Briefly, 5-day-old turkey poults were orally gavaged with $100 \mathrm{mg} / \mathrm{kg}$ of body weight NTZ once daily for 4 days prior to oral infection with intestinal filtrate containing between $10^{12}$ and $10^{13}$ genome copy units of turkey astrovirus strain 2 (TAstV-2) in $500 \mu 1$ phosphate-buffered saline (PBS) and then for 3 days post-infection (Figure 5-6A). The dose of NTZ used to treat the turkey poults was selected based on previously published studies of NTZ treatment in mice. ${ }^{403-405}$ Stool samples were scored daily by four blinded volunteers as previously described. ${ }^{42}$ The scoring scale ranged from 1 to 4 based on color and consistency, with scores of 3 and 4 being considered diarrhea. Stool samples were also collected to quantitate viral titers. Although the difference was not statistically significant, NTZ treatment resulted in fewer poults with clinical disease (Figure 5-6B). This likely results from the significant reduction $(\mathrm{P}=0.005)$ in stool viral loads throughout the course of infection (Figure 5-6C). The poults also showed no adverse symptoms from receiving NTZ alone. Excitingly, this gives evidence NTZ may be an effective therapeutic for astrovirus-induced diarrheal disease.

\section{Discussion}

Our study provides the first evidence of an effective antiviral for astrovirus infection. We showed that NTZ is broadly protective against multiple classical HAstV serotypes and reduces the production of dsRNA during infection in vitro with an $\mathrm{EC}_{50}$ of approximately $1.47 \mu \mathrm{M}$. Additionally, we showed the potential NTZ has as a clinical therapeutic by its ability to reduce viral shedding and clinical disease in our symptomatic turkey poult model.

Nitazoxanide [2-acetyloxy-N-(5-nitro-2-thiazolyl) benzamide] (Alinia; Romark Laboratories) is a thiazolide compound for treatment of both intestinal protozoal and helminthic infections, specifically Giardia lamblia and Cryptosporidium parvum. ${ }^{406}$ Recently, this compound has been shown to have antiviral properties as well. The use of NTZ in vitro has been reported as an antiviral against influenza virus, ${ }^{407}$ rotavirus, ${ }^{408}$ norovirus,${ }^{409}$ Japanese encephalitis virus (JEV), ${ }^{404}$ rubella virus, ${ }^{410}$ Zika virus, ${ }^{411}$ hepatitis $\mathrm{C}$ virus, ${ }^{412}$ and hepatitis B virus. ${ }^{413}$ Successful clinical trials have demonstrated its effectiveness in treating influenza virus, ${ }^{414}$ norovirus and rotavirus, ${ }^{409,415,416}$ hepatitis B virus, ${ }^{417}$ and hepatitis C virus. ${ }^{412,418}$ Its mechanism of action against protozoa is due to its interference with pyruvate:ferredoxin oxidoreductase (PFOR) enzyme-dependent electron transfer reactions. ${ }^{419}$ While its antiviral action is currently unknown, research suggests it may be through the induction of the interferon response via activation of protein kinase $\mathrm{R}$ or disruption of the unfolded protein response. ${ }^{407}$ We show that NTZ disrupts astrovirus infection early in the replication cycle, causing a significant decrease in the production of dsRNA. The inhibition by NTZ at an early stage of infection was also seen with JEV. ${ }^{404}$ 
A.

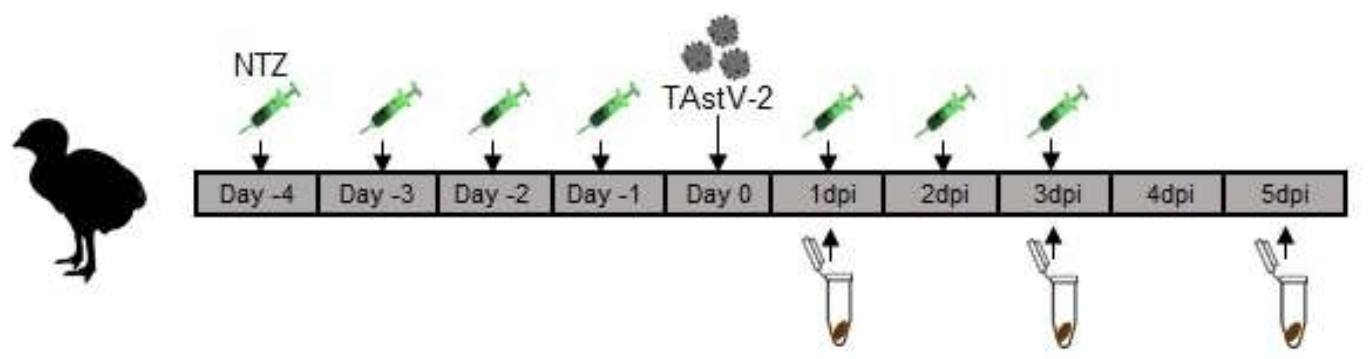

B.
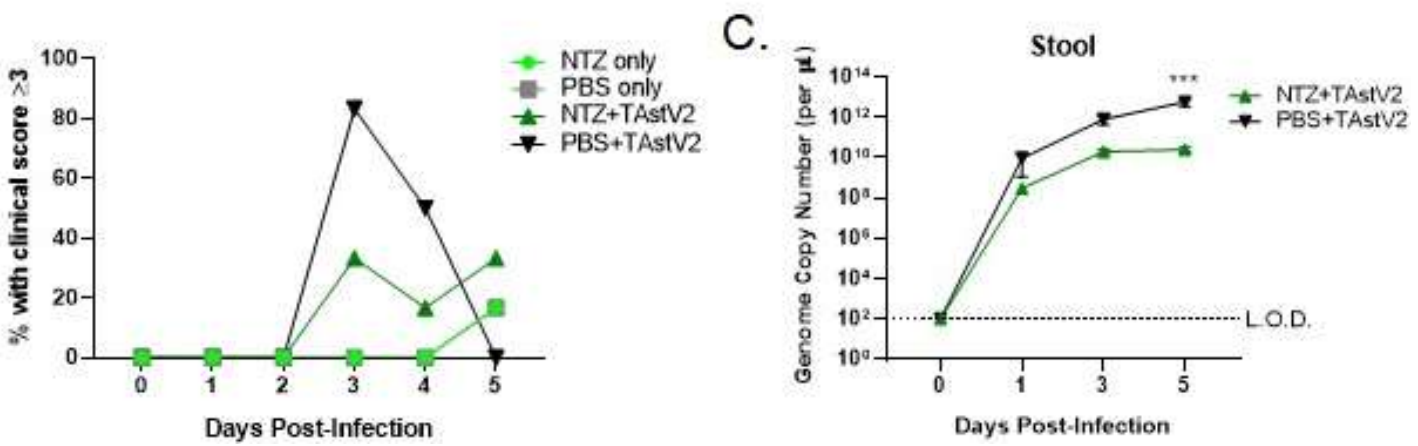

Figure 5-6. Nitazoxanide Reduces Clinical Symptoms and Viral Titers in Turkey Poults.

Turkey poults ( $\mathrm{n}=6$ per group) were infected with turkey astrovirus (TAstV-2) from intestinal filtrate. For 4 days prior to infection and 3 days post-infection, poults were treated with NTZ. Poults were monitored for clinical score daily, and stool samples were collected to measure viral RNA titers every other day. (A) Percentages of poults with clinical scores of 3 or higher in the treatment groups indicated in the key. PBS, no antiviral treatment. (B) Viral RNA titers of stool samples collected from infected poults with or without NTZ treatment. All error bars indicate standard errors of the means, and dashed line represents the limit of detection. Asterisks show statistical significance as measured by two-way ANOVA as follows: *, P $0.05 ; * *$, P 0.01 ; *** , P 0.001 . 
Recent research has shown that thiazolides upregulate type I and II IFN, ${ }^{402}$ which modulate the immune system, and this could be how NTZ creates a broadly antiviral state. However, the rapid kinetics with which NTZ inhibits HAstV replication

(Figure 5-3) suggests that the induction of IFN is not responsible. Studies to uncover the exact mechanism will be difficult until further tools are available to investigate astrovirus replication.

Human astroviruses are classified into genotypes, and within the classical human genotype, comprising the genus Mamastrovirus 1 (MAstV1), strains are further divided into serotypes (HAstV-1 to -8) based on their antigenicities and genetic differences in the complete capsid sequences. ${ }^{127}$ These genetic differences between astrovirus serotypes can confer differences in replication kinetics and symptom severity. ${ }^{420}$ Thus, finding a compound that broadly inhibits astroviruses across genotypes and serotypes is crucial. We found that NTZ is broadly protective across multiple HAstV serotypes, including the dominant strain worldwide, HAstV-1. ${ }^{152}$ Future studies will assess the ability of NTZ to inhibit the replication of the nonclassical HAstV genotypes, MLB and VA (MAstV6, -8, and -9), which have been associated with rare cases of encephalitis or meningitis in immunocompromised patients, ${ }^{87}$ although there has been 1 case of classical HAstVassociated encephalitis.

To evaluate efficacy in vivo, we turned to the turkey poult model. Turkey poults exhibit age-dependent diarrhea similar to that in humans when infected with TAstV, making them the only clinically relevant small animal model for astrovirus identified to date. ${ }^{39,119}$ We found a significant decrease $(\mathrm{P}=0.005)$ in stool viral titers, with NTZtreated poults having nearly $2 \log$ less virus at 5 days post-infection. We also showed that viral titers began to plateau in the NTZ-treated poults at 5 days post-infection, while untreated poults still showed increasing titers. This suggests NTZ treatment may lead to faster clearance of the virus. The decreased viral titers were associated with fewer poults having clinical disease. It is not surprising that NTZ did not completely prevent clinical disease. Viral titers are reduced but not completely inhibited. Furthermore, we demonstrated that administration of viral capsid alone is sufficient to induce diarrhea in turkey poults. ${ }^{42}$ Therefore, NTZ treatment may not be able to fully inhibit AstV-induced diarrhea. Finally, we administered between $10^{12}$ and $10^{13}$ genome copy units to each poult. While there have been reports of virus shedding in humans at this level, ${ }^{420}$ it is a large viral dose that may not be representative of natural infection. Overall, this work provides the first evidence that NTZ may be an effective antiviral option against human classical HAstV and turkey astrovirus infections. 


\section{CHAPTER 6. CONCLUSIONS AND FUTURE DIRECTIONS}

Previous research has shown that astrovirus pathogenesis is unique in that it disrupts tight junctions to compromise barrier integrity. ${ }^{349}$ The studies in this dissertation extend our initial work to demonstrate that this disruption of cellular junctions is just the beginning; we show that astrovirus infection induces epithelial-mesenchymal transition (EMT). Beginning with the loss of cell-cell contacts and a transcriptional upregulation of EMT related genes at 8 hours post-infection (hpi), astrovirus infected cells display disrupted cell polarity and express mesenchymal proteins like vimentin by 24 hpi. While the exact mechanism by which HAstV induces EMT has yet to be determined, we have shown this process is dependent on productive viral replication. In addition, these studies have identified an FDA-approved antiviral that effectively inhibits astrovirus replication. Nitazoxanide blocks astrovirus replication in vitro with an $\mathrm{EC}_{50}$ of approximately $1.47 \mu \mathrm{M}$ and is effective against a broad range of human astrovirus serotypes. Through the course of performing these studies, more interesting findings were revealed that will require further investigation.

Soon after identifying the phenotypic changes associated with EMT, we sought to determine the mechanism(s). We performed a microarray and gene set enrichment analysis, between HAstV-infected and uninfected Caco-2 cells to examine which genes were modulated by infection. The results from this microarray showed several cytokine pathways were upregulated, including TNF- $\alpha$ and TGF- $\beta$ related signaling. We initially hypothesized that astrovirus-binding and/or replication, resulted in the secretion of a cellular factor that induced EMT. Pro-inflammatory cytokines like TNF- $\alpha$ and IL-6 are known inducers of EMT. ${ }^{421-423}$ Despite previous work in the lab demonstrating a lack of cytokine or chemokine production in the first 24 hours of infection, supernatants from HAstV infected cells were collected at 18, 24, and 48 hpi. These supernatants were UVinactivated and placed on naïve Caco-2 cells to determine if a secreted factor was causing increased barrier permeability and induction of EMT. None of the collected supernatants caused a drop in TER (Figure A-1A). Supernatants from HAstV and mock infected cells were then tested for the pro-inflammatory cytokine, TNF- $\alpha$, by ELISA because it was a top upregulated hit in our microarray analysis. While, neither the supernatants from HAstV or mock infected cells had significant levels of TNF- $\alpha$, the viral stocks did contain trace levels of TNF- $\alpha$ (Figure A-1B). This discrepancy is likely due to experimental conditions. Viral stocks are prepared by infecting post-confluent Caco-2 cells in the presence of $10 \mu \mathrm{g} / \mathrm{ml}$ porcine trypsin, allowing for multiple round of infection, and collecting the supernatant 4 days later. This contrasts to our experimental studies which are conducted between 8 and 48 hpi without trypsin allowing for only a single round of viral replication. This suggests that at least TNF- $\alpha$ and potentially other cellular factors may be released during multi-cycle replication and could be present in our viral stocks. Thus, the viral stock was purified, shown to be free of TNF- $\alpha$ and EMT assays were performed. Purified viral stocks, free of detectable TNF- $\alpha$ still induced EMT (Figure A-1C), suggesting neither TNF- $\alpha$, some other secreted cytokine, nor possible stock contaminants were responsible for HAstV-induced EMT. 
Another signaling pathway shown to be upregulated by our microarray analysis was TGF- $\beta$. However, an increase in TGF- $\beta$ mRNA does not always equate to increased TGF- $\beta$ activity. When TGF- $\beta$ is produced, it is in conjunction with a latency-associated protein (LAP) that prevents TGF- $\beta$ from binding to its receptor. The LAP must be removed in order to activate TGF- $\beta$. Using the PAI biological assay, we observed an increase in active TGF- $\beta$ during HAstV infection (Figure 4-5A). Latent TGF- $\beta$ can be activated by a number of mechanisms including proteases, reactive oxygen, heat, acidic $\mathrm{pH}$, engagement with integrins and thrombospondin-1 (TSP-1). ${ }^{424}$ To determine what was activating TGF- $\beta$, conditioned media containing latent TGF- $\beta$ from Caco- 2 cells was incubated with purified HAstV as well as known activators of TGF- $\beta$ (heat, $1 \mathrm{~N} \mathrm{HCl}$, and TSP-1). We discovered that purified HAstV can directly activate latent. While HAstV is not as potent of an activator as heat or $\mathrm{HCl}$, it did activate TGF- $\beta$ above background levels (Figure B-1). Although collectively our data indicates that TGF- $\beta$ is likely not the main mechanism utilized by HAstV to induce EMT, these results suggest is still an important factor in astrovirus pathogenesis. TGF- $\beta$ is likely very important in vivo in creating an immune suppressive microenvironment. Additionally, it may be important in inducing EMT in bystander cells at later times post-infection. Future studies will examine how HAstV is directly activating TGF- $\beta$ and how TGF- $\beta$ affects astrovirus replication.

Since our data suggested TGF- $\beta$ was not the main mechanism inducing EMT, we investigated other less common inducers of EMT like miRNAs. Our lab showed several cellular miRNAs were modulated during HAstV infection, but none of these were involved in the increased barrier permeability during infection. We then identified three miRNAs encoded in the HAstV genome. MiR-487a, a miRNA encoded into the capsid portion of the HAstV genome, impacted HAstV-induced barrier permeability as well as viral replication. Interestingly, miR-487a has been reported to be involved in TGF- $\beta$ mediated EMT. ${ }^{425}$ We wanted to determine whether miR-487a played a role in HAstVinduced EMT. We screened clinical isolates, our lab has collected through longitudinal cohort studies of the St. Jude patient, for sequence homology to miR-487a and three isolates with varying degrees of similarity to miR-487a were identified. Caco- 2 cells were infected with these three isolates and the characteristics of HAstV-induced EMT were examined. One isolate in particular, CI-110, that had very little sequence homology to miR-487a, exhibited delayed barrier permeability kinetics, and higher expression of Ecadherin than the other isolates and the lab adapted HAstV-1 (Figure C-1). This finding was extremely intriguing, as other viruses have been shown to use miRNAs to induce EMT. ${ }^{391-393}$ However, more investigations are needed to validate this finding.

Following the examination of the mechanism of HAstV-induced EMT, we asked what the role of EMT is in the context of astrovirus pathogenesis. Both my studies and those previously published ${ }^{349}$ showed that occludin was the first junctional protein to be disrupted during HAstV infection (Figure 4-1). Using cells with occludin knocked down by $75 \%$ via shRNA (a generous gift from Dr. Carolyn Coyne) (Figure D-1A), we sought to determine if the disruption of occludin was necessary for HAstV replication and EMT. When occludin levels are reduced, the percent of HAstV infected cells in the monolayer increases (Figure D-1B, C). I hypothesize that in the absence of occludin, the receptor used by HAstV may be more accessible and allow a greater percentage of cells to be 
infected. Despite the difference in infection between the knockdown cells and Caco-2, there was no difference in the amount of virus released into the supernatant suggesting a possible role for occludin in viral egress. Studies have shown that occludin reorganization is mediated by phosphorylation. ${ }^{426-428}$ I hypothesize the ERK signaling cascade, which our lab has showed was activated within 30 minutes of HAstV infection, ${ }^{370}$ and which was necessary for HAstV-induced EMT (Figure 4-8), could mediate phosphorylation of occludin and its subsequent reorganization. Future work will solidify the role of occludin in astrovirus pathogenesis and EMT.

EMT may also contribute to pathogenesis by permitting astrovirus to cross the intestinal epithelium and cause systemic infection. EMT disrupts barrier integrity and creates gaps that pathogens can use to gain access to the body. Disseminated astrovirus infections have been reported in animals for decades. More recently novel HAstV genotypes were reported to be associated with cases of encephalitis and meningitis. In the past year, two St. Jude patients required treatment for HAstV-VA1-induced encephalitis. Since there have been only a few reported cases of HAstV-VA1 infection and even fewer with positive outcomes ( $60 \%$ fatality rate; Table 1-1), physicians had very little information on effective treatments. Based on our antiviral work, our clinical colleagues administered NTZ to both patients. Both survived their infections. HAstV encephalitic patients have also been administered intravenous IgG (IVIG), as this has been proposed as a treatment option for HAstV. We questioned whether commercially available IVIG had HAstV-VA1 specific antibodies and if it could confer neutralization of the virus, to determine if the treatment should be continued. ELISA assay demonstrated nine different lots of IVIG all had equal amount of HAstV-VA1 specific antibodies. Surprisingly, there were significantly more HAstV-VA1 antibodies than HAstV-1 (Figure E-1A, B). Studies of HAstV prevalence have always suggested that HAstV-1 is the most common HAstV infection. ${ }^{152,155}$ These results and more recent studies would suggest that HAstV-VA1 may be just as prevalent, or that antibodies to HAstV-VA1 are more long-lasting. When examining the neutralization of these antibodies, one lot showed some neutralization capacity at a dilution of 1:50 (Figure E-1C, D). It appears that these antibodies may be able to block entry, since there was a reduction in cell bound genome copies but not in the supernatant. Given these results, IVIG may serve as another treatment option for severe HAstV infection. However, due to the cost of IVIG treatments, it may not be feasible in all cases. Although we have not shown NTZ inhibits novel HAstV genotypes, I hypothesize it would inhibit these genotypes as well, because NTZ blocked the formation of dsRNA, a necessity among all astroviruses. We now have HAstV-VA1 propagated in the lab and will investigate this novel genotype in the future.

While EMT is clearly embedded as part of astrovirus pathogenesis, we do need to question why this process is happening and whether it is a benefit to the virus, the host, or both. As discussed in Chapter 4, EMT is induced by a handful of viruses, the majority of which are oncogenic. With these viruses, EMT is a means to create secondary metastases and spread to new areas of the body. However, in the case of the non-oncogenic viruses the need of EMT is less obvious. It has been suggested for both TGEV and rhinovirus that the induction of EMT and the associated increase in TGF- $\beta$ create an immunosuppressive microenvironment. ${ }^{340,341}$ This could be the case for HAstV since a 
lack of inflammation is a hallmark of astrovirus infection. Additionally, we observed cells that appeared to be extruded out of the cell monolayer during astrovirus infection (Figure 4-4A). This made us question if HAstV-induced EMT was part of the wound healing process. This would make sense as EMT is heavy involved in wound repair and if HAstV-induced EMT were to be categorized into one of the three types of EMT discussed in Chapter 2, it most closely resembles type II EMT. Whether EMT benefits HAstV or the host is still a difficult question to answer with the data my studies have generated. On the one hand, creating an immunosuppressive microenvironment probably benefits the virus more than host. On the other hand, closing gaps in the intestinal epithelium is more of a necessity for the host. Throughout these studies, very high MOI was used during infections to get as many cells in the monolayer infected as possible. While RNA was extracted from the entire monolayer, there was never $100 \%$ infection. This leaves the possibility of non-infected bystander cells responding to neighboring cells infected with HAstV. We saw glimpses of this in staining for SMAD3 (Figure 4-5), where SMAD3 translocated to the nucleus mainly in non-infected bystander cells. By using either cell sorting of infected cells or through single-cell transcriptomics, it would be possible to determine if transcriptional regulation of EMT was occurring in infected cells, non-infected cells, or both. I would then hypothesize if EMT is occurring in infected cells, the process is a benefit to the virus; if it is occurring in bystander cells, the process is a result of the virus but a benefit to the host.

In addition to performing cell sorting or single cell transcriptomics, further investigation into miR-487a as a possible mechanism for HAstV-induced EMT needs to be conducted. First, we need to show miR-487a is expressed during infection. Sequence homology to this miRNA was identified, but that does not mean it is actually expressed and processed in our system. Additionally, the clinical isolates used were different serotypes of HAstV, meaning there are other genetic differences in these viruses aside from their sequence homology to miR-487a. Ideally, to determine if miR-487a is involved in HAstV-induced EMT, we would use site directed mutagenesis to alter the miRNA sequence without modifying the remainder of the capsid genome or structure. Finally, we would need to identify the target of miR-487a whose down-regulation would be responsible for inducing EMT. These measures will allow us to determine the role of miR-487a in HAstV-induced EMT. Another clinically relevant aspect of this study that needs further investigation is whether the novel genotypes induce EMT. These genotypes are at the root of the most devastating HAstV disease whose prevalence is much more common than once thought and yet so little is known about them. By determining if these genotypes induce EMT, we can gain a little more insight into what separates them from other HAstV, while possibly generating more therapeutic targets to treat systemic infection.

In conclusion, the studies performed in this dissertation have provided fundamental new research on astrovirus pathogenesis as well as new treatment options. In addition, the work and outcomes outlined above provide a platform for further research to build upon and create new areas of astrovirus research. The finding that a non-oncogenic, enteric virus such as HAstV induces EMT will hopefully lead to new investigations of 
non-oncogenic viruses and novel areas of research into the pathophysiology of the gastrointestinal tract by viruses. 


\section{LIST OF REFERENCES}

1. Appleton, H. \& Higgins, P. G. Viruses and gastroenteritis in infants. The Lancet (1975) doi: 10.1016/s0140-6736(75)92581-7.

2. Madeley, C. R., Cosgrove, B. P. \& Bell, E. J. Stool viruses in babies in Glasgow: 2. Investigation of normal newborns in hospital. Epidemiol. Infect. 81, 285-294 (1978) doi: $10.1017 / \mathrm{s} 0022172400025122$.

3. Madeley, C. R. \& Cosgrove, B. P. Letter: $28 \mathrm{~nm}$ particles in faeces in infantile gastroenteritis. Lancet Lond. Engl. 2, 451-452 (1975) doi: 10.1016/s0140$\underline{6736(75) 90858-2 .}$.

4. Monroe, S. S., Jiang, B., Stine, S. E., Koopmans, M. \& Glass, R. I. Subgenomic RNA sequence of human astrovirus supports classification of Astroviridae as a new family of RNA viruses. J. Virol. 67, 3611-3614 (1993).

5. Ninth Report of the International Committee on Taxonomy of Viruses. (Elsevier, 2012).

6. Finkbeiner, S. R. et al. Human stool contains a previously unrecognized diversity of novel astroviruses. Virol. J. 6, 161 (2009) doi: 10.1186/1743-422X-6-161.

7. Finkbeiner, S. R. et al. Identification of a novel astrovirus (astrovirus VA1) associated with an outbreak of acute gastroenteritis. J. Virol. 83, 10836-10839 (2009) doi: 10.1128/JVI.00998-09.

8. Finkbeiner, S. R., Le, B. M., Holtz, L. R., Storch, G. A. \& Wang, D. Detection of newly described astrovirus MLB1 in stool samples from children. Emerg. Infect. Dis. 15, 441-444 (2009) doi: 10.3201/eid1503.081213.

9. Finkbeiner, S. R., Kirkwood, C. D. \& Wang, D. Complete genome sequence of a highly divergent astrovirus isolated from a child with acute diarrhea. Virol. J. 5, 117 (2008) doi: $\underline{10.1186 / 1743-422 X-5-117 .}$

10. Jiang, H. et al. Comparison of novel MLB-clade, VA-clade and classic human astroviruses highlights constrained evolution of the classic human astrovirus nonstructural genes. Virology 436, 8-14 (2013) doi: 10.1016/j.virol.2012.09.040.

11. Kapoor, A. et al. Multiple novel astrovirus species in human stool. J. Gen. Virol. 90, 2965-2972 (2009) doi: 10.1099/vir.0.014449-0.

12. Finkbeiner, S. R. et al. Metagenomic Analysis of Human Diarrhea: Viral Detection and Discovery. PLOS Pathog. 4, e1000011 (2008) doi: 10.1371/journal.ppat.1000011. 
13. Meyer, C. T. et al. Prevalence of classic, MLB-clade and VA-clade Astroviruses in Kenya and The Gambia. Virol. J. 12, (2015) doi: 10.1186/s12985-015-0299-z.

14. Méndez, E., Murillo, A., Velazquez, R., Burnham, A. \& Arias, C. F. Replication Cycle of Astroviruses. in Astrovirus Research: Essential Ideas, Everyday Impacts, Future Directions 19-45 (Springer Science+Business Media, 2013).

15. Willcocks, M. M. \& Carter, M. J. Identification and sequence determination of the capsid protein gene of human astrovirus serotype 1. FEMS Microbiol. Lett. 114, 1-7 (1993) doi: 10.1016/0378-1097(93)90133-m.

16. Al-Mutairy, B., Walter, J. E., Pothen, A. \& Mitchell, D. K. Genome Prediction of Putative Genome-Linked Viral Protein (VPg) of Astroviruses. Virus Genes 31, 21-30 (2005) doi: 10.1007/s11262-004-2196-1.

17. Fuentes, C., Guix, S., Bosch, A. \& Pintó, R. M. The C-Terminal nsP1a Protein of Human Astrovirus Is a Phosphoprotein That Interacts with the Viral Polymerase ${ }^{\nabla}$. J. Virol. 85, 4470-4479 (2011) doi: 10.1128/JVI.01515-10.

18. Geigenmüller, U., Chew, T., Ginzton, N. \& Matsui, S. M. Processing of Nonstructural Protein 1a of Human Astrovirus. J. Virol. 76, 2003-2008 (2002) doi: $\underline{10.1128 / j v i .76 .4 .2003-2008.2002 . ~}$

19. Guix, S., Caballero, S., Bosch, A. \& Pintó, R. M. Human astrovirus C-terminal nsP1a protein is involved in RNA replication. Virology 333, 124-131 (2005) doi: 10.1016/j.virol.2004.12.023.

20. Kiang, D. \& Matsui, S. M. Proteolytic processing of a human astrovirus nonstructural protein. J. Gen. Virol. 83, 25-34 (2002) doi: 10.1099/0022-1317$\underline{83-1-25 .}$

21. Lewis, T. L., Greenberg, H. B., Herrmann, J. E., Smith, L. S. \& Matsui, S. M. Analysis of astrovirus serotype 1 RNA, identification of the viral RNA-dependent RNA polymerase motif, and expression of a viral structural protein. J. Virol. 68, 77-83 (1994).

22. Méndez, E., Salas-Ocampo, M. P. E., Munguía, M. E. \& Arias, C. F. Protein Products of the Open Reading Frames Encoding Nonstructural Proteins of Human Astrovirus Serotype 8. J. Virol. 77, 11378-11384 (2003) doi: 10.1128/jvi.77.21.11378-11384.2003.

23. Jiang, B., Monroe, S. S., Koonin, E. V., Stine, S. E. \& Glass, R. I. RNA sequence of astrovirus: distinctive genomic organization and a putative retrovirus-like ribosomal frameshifting signal that directs the viral replicase synthesis. Proc. Natl. Acad. Sci. U. S. A. 90, 10539-10543 (1993) doi: 10.1073/pnas.90.22.10539. 
24. Marczinke, B. et al. The human astrovirus RNA-dependent RNA polymerase coding region is expressed by ribosomal frameshifting. J. Virol. 68, 5588-5595 (1994).

25. Risco, C., Carrascosa, J. L., Pedregosa, A. M., Humphrey, C. D. \& SánchezFauquier, A. Ultrastructure of human astrovirus serotype 2. J. Gen. Virol. 76 ( Pt 8), 2075-2080 (1995) doi: 10.1099/0022-1317-76-8-2075.

26. Dong, J., Dong, L., Méndez, E. \& Tao, Y. Crystal structure of the human astrovirus capsid spike. Proc. Natl. Acad. Sci. U. S. A. 108, 12681-12686 (2011) doi: $\underline{10.1073 / \text { pnas.1104834108. }}$.

27. Espinosa-Hernández, W. et al. PTB Binds to the 3' Untranslated Region of the Human Astrovirus Type 8: A Possible Role in Viral Replication. PLOS ONE 9, e113113 (2014) doi: 10.1371/journal.pone.0113113.

28. York, R. L. et al. Structural, Mechanistic, and Antigenic Characterization of the Human Astrovirus Capsid. J. Virol. 90, 2254-2263 (2016) doi: 10.1128/JVI.02666-15.

29. Bogdanoff, W. A., Perez, E. I., López, T., Arias, C. F. \& DuBois, R. M. Structural Basis for Escape of Human Astrovirus from Antibody Neutralization: Broad Implications for Rational Vaccine Design. J. Virol. 92, (2018) doi: 10.1128/JVI.01546-17.

30. DuBois, R. M. et al. Crystal structure of the avian astrovirus capsid spike. $J$. Virol. 87, 7853-7863 (2013) doi: 10.1128/JVI.03139-12.

31. Dryden, K. A. et al. Immature and mature human astrovirus: structure, conformational changes, and similarities to hepatitis E virus. J. Mol. Biol. 422, 650-658 (2012) doi: 10.1016/j.jmb.2012.06.029.

32. Baños-Lara, M. del R. \& Mendez, E. Role of individual caspases induced by astrovirus on the processing of its structural protein and its release from the cell through a non-lytic mechanism. Virology 401, 322-332 (2010) doi: 10.1016/j.virol.2010.02.028.

33. Méndez, E., Salas-Ocampo, E. \& Arias, C. F. Caspases mediate processing of the capsid precursor and cell release of human astroviruses. J. Virol. 78, 8601-8608 (2004) doi: 10.1128/JVI.78.16.8601-8608.2004.

34. Bass, D. M. \& Qiu, S. Proteolytic Processing of the Astrovirus Capsid. J. Virol. 74, 1810-1814 (2000) doi: 10.1128/jvi.74.4.1810-1814.2000.

35. Arias, C. F. \& DuBois, R. M. The Astrovirus Capsid: A Review. Viruses 9, (2017) doi: 10.3390/v9010015. 
36. Sanchez-Fauquier, A. et al. Characterization of a human astrovirus serotype 2 structural protein (VP26) that contains an epitope involved in virus neutralization. Virology 201, 312-320 (1994) doi: 10.1006/viro.1994.1296.

37. Bonaparte, R. S. et al. Human Astrovirus Coat Protein Inhibits Serum Complement Activation via C1, the First Component of the Classical Pathway. $J$. Virol. 82, 817-827 (2008) doi: 10.1128/JVI.01847-07.

38. Hair, P. S. et al. Human astrovirus coat protein binds C1q and MBL and inhibits the classical and lectin pathways of complement activation. Mol. Immunol. 47, 792-798 (2010) doi: 10.1016/j.molimm.2009.10.006.

39. Koci, M. D. et al. Astrovirus induces diarrhea in the absence of inflammation and cell death. J. Virol. 77, 11798-11808 (2003) doi: 10.1128/jvi.77.21.11798$\underline{11808.2003 .}$

40. Sebire, N. J. et al. Pathology of astrovirus associated diarrhoea in a paediatric bone marrow transplant recipient. J. Clin. Pathol. 57, 1001-1003 (2004) doi: 10.1136/jcp.2004.017178.

41. Moser, L. A., Carter, M. \& Schultz-Cherry, S. Astrovirus Increases Epithelial Barrier Permeability Independently of Viral Replication. J. Virol. 81, 1193711945 (2007) doi: 10.1128/JVI.00942-07.

42. Meliopoulos, V. A. et al. Oral Administration of Astrovirus Capsid Protein Is Sufficient To Induce Acute Diarrhea In Vivo. mBio 7, (2016) doi: 10.1128/mBio.01494-16.

43. Brinker, J. P., Blacklow, N. R. \& Herrmann, J. E. Human astrovirus isolation and propagation in multiple cell lines. Arch. Virol. 145, 1847-1856 (2000) doi: $\underline{10.1007 / \mathrm{s} 007050070060 .}$.

44. Donelli, G., Superti, F., Tinari, A. \& Marziano, M. L. Mechanism of astrovirus entry into Graham 293 cells. J. Med. Virol. 38, 271-77 (1992) doi:

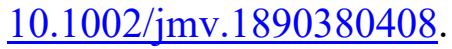

45. Méndez, E. et al. Characterization of human astrovirus cell entry. J. Virol. 88, 2452-2460 (2014) doi: 10.1128/JVI.02908-13.

46. Caballero, S., Guix, S., Ribes, E., Bosch, A. \& Pintó, R. M. Structural requirements of astrovirus virus-like particles assembled in insect cells. J. Virol. 78, 13285-13292 (2004) doi: 10.1128/JVI.78.23.13285-13292.2004.

47. Méndez, E., Aguirre-Crespo, G., Zavala, G. \& Arias, C. F. Association of the astrovirus structural protein VP90 with membranes plays a role in virus morphogenesis. J. Virol. 81, 10649-10658 (2007) doi: 10.1128/JVI.00785-07. 
48. Guix, S., Caballero, S., Bosch, A. \& Pintó, R. M. C-terminal nsP1a protein of human astrovirus colocalizes with the endoplasmic reticulum and viral RNA. $J$. Virol. 78, 13627-13636 (2004) doi: 10.1128/JVI.78.24.13627-13636.2004.

49. Gibson, C. A., Chen, J., Monroe, S. A. \& Denison, M. R. Expression and processing of nonstructural proteins of the human astroviruses. Adv. Exp. Med. Biol. 440, 387-391 (1998) doi: 10.1007/978-1-4615-5331-1_50.

50. Fuentes, C., Bosch, A., Pintó, R. M. \& Guix, S. Identification of Human Astrovirus Genome-Linked Protein (VPg) Essential for Virus Infectivity. J. Virol. 86, 10070-10078 (2012) doi: 10.1128/JVI.00797-12.

51. Velázquez-Moctezuma, R., Baños-Lara, M. del R., Acevedo, Y. \& Méndez, E. Alternative cell lines to improve the rescue of infectious human astrovirus from a cDNA clone. J. Virol. Methods 179, 295-302 (2012) doi: 10.1016/j.jviromet.2011.11.005.

52. Monroe, S. S. et al. Temporal synthesis of proteins and RNAs during human astrovirus infection of cultured cells. J. Virol. 65, 641-648 (1991).

53. Sztuba-Solińska, J., Stollar, V. \& Bujarski, J. J. Subgenomic messenger RNAs: mastering regulation of $(+)$-strand RNA virus life cycle. Virology 412, 245-255 (2011) doi: 10.1016/j.virol.2011.02.007.

54. Murillo, A., Vera-Estrella, R., Barkla, B. J., Méndez, E. \& Arias, C. F. Identification of Host Cell Factors Associated with Astrovirus Replication in Caco-2 Cells. J. Virol. 89, 10359-10370 (2015) doi: 10.1128/JVI.01225-15.

55. Moser, L. A. \& Schultz-Cherry, S. Suppression of astrovirus replication by an ERK1/2 inhibitor. J. Virol. 82, 7475-7482 (2008) doi: 10.1128/JVI.02193-07.

56. Casorla-Pérez, L. A., López, T., López, S. \& Arias, C. F. The ubiquitinproteasome system is necessary for the efficient replication of human astrovirus. J. Virol. (2017) doi:10.1128/JVI.01809-17.

57. Banos-Lara, M. del R. \& Méndez, E. Role of individual caspases induced by astrovirus on the processing of its structural protein and its release from the cell through a non-lytic mechanism. Virology 401, 322-332 (2010).

58. Marvin, S. A. et al. Type I Interferon Response Limits Astrovirus Replication and Protects against Increased Barrier Permeability In Vitro and In Vivo. J. Virol. 90, 1988-1996 (2015) doi: 10.1016/j.virol.2010.02.028. 
59. Nighot, P. K., Moeser, A., Ali, R. A., Blikslager, A. T. \& Koci, M. D. Astrovirus infection induces sodium malabsorption and redistributes sodium hydrogen exchanger expression. Virology 401, 146-154 (2010) doi: 10.1016/j.virol.2010.02.004.

60. Torres-Flores, J. M. \& Arias, C. F. Tight Junctions Go Viral! Viruses 7, 51455154 (2015) doi: 10.3390/v7092865.

61. France, M. M. \& Turner, J. R. The mucosal barrier at a glance. J. Cell Sci. 130, 307-314 (2017) doi: 10.1242/jcs.193482.

62. Gray, E. W., Angus, K. W. \& Snodgrass, D. R. Ultrastructure of the small intestine in astrovirus-infected lambs. J. Gen. Virol. 49, 71-82 (1980) doi: 10.1099/0022-1317-49-1-71.

63. Snodgrass, D. R., Angus, K. W., Gray, E. W., Menzies, J. D. \& Paul, G. Pathogenesis of diarrhoea caused by astrovirus infections in lambs. Arch. Virol. 60, 217-226 (1979) doi: 10.1007/BF01317493.

64. Woode, G. N., Pohlenz, J. F., Gourley, N. E. \& Fagerland, J. A. Astrovirus and Breda virus infections of dome cell epithelium of bovine ileum. J. Clin. Microbiol. 19, 623-630 (1984).

65. Janowski, A. B., Klein, R. S. \& Wang, D. Differential In Vitro Infection of Neural Cells by Astroviruses. mBio 10, (2019) doi: 10.1128/mBio.01455-19.

66. Janowski, A. B., Bauer, I. K., Holtz, L. R. \& Wang, D. Propagation of Astrovirus VA1, a Neurotropic Human Astrovirus, in Cell Culture. J. Virol. 91, (2017) doi: 10.1128/JVI.00740-17.

67. Vu, D.-L., Bosch, A., Pintó, R. M., Ribes, E. \& Guix, S. Human Astrovirus MLB Replication In Vitro: Persistence in Extraintestinal Cell Lines. J. Virol. 93, (2019) doi: 10.1128/JVI.00557-19.

68. Janowski, A. B. \& Wang, D. Infection and Propagation of Astrovirus VA1 in Cell Culture. Curr. Protoc. Microbiol. 52, e73 (2019) doi: 10.1002/cpmc. 73.

69. Kolawole, A. O. et al. Astrovirus replication in human intestinal enteroids reveals multi-cellular tropism and an intricate host innate immune landscape. PLoS Pathog. 15, e1008057 (2019) doi: 10.1371/journal.ppat.1008057.

70. Cortez, V. et al. Astrovirus infects actively secreting goblet cells and alters the gut mucus barrier. Nat. Commun. 11, 2097 (2020) doi: 10.1038/s41467-020-15999-y.

71. Guix, S. et al. Type I interferon response is delayed in human astrovirus infections. PloS One 10, e0123087 (2015) doi: 10.1371/journal.pone.0123087. 
72. Koci, M. D., Kelley, L. A., Larsen, D. \& Schultz-Cherry, S. Astrovirus-Induced Synthesis of Nitric Oxide Contributes to Virus Control during Infection. J. Virol. 78, 1564-1574 (2004) doi: 10.1128/jvi.78.3.1564-1574.2004.

73. Qureshi, M. A., Saif, Y. M., Heggen-Peay, C. L., Edens, F. W. \& Havenstein, G. B. Induction of functional defects in macrophages by a poult enteritis and mortality syndrome-associated turkey astrovirus. Avian Dis. 45, 853-861 (2001).

74. Meyerhoff, R. R., Nighot, P. K., Ali, R. A., Blikslager, A. T. \& Koci, M. D. Characterization of turkey inducible nitric oxide synthase and identification of its expression in the intestinal epithelium following astrovirus infection. Comp. Immunol. Microbiol. Infect. Dis. 35, 63-69 (2012) doi: 10.1016/j.cimid.2011.10.002.

75. Kurtz, J. \& Lee, T. Astrovirus gastroenteritis age distribution of antibody. Med. Microbiol. Immunol. (Berl.) 166, 227-230 (1978) doi: 10.1007/BF02121154.

76. Kurtz, J. B., Lee, T. W., Craig, J. W. \& Reed, S. E. Astrovirus infection in volunteers. J. Med. Virol. 3, 221-230 (1979) doi: 10.1002/jmv.1890030308.

77. Midthun, K. et al. Characterization and seroepidemiology of a type 5 astrovirus associated with an outbreak of gastroenteritis in Marin County, California. J. Clin. Microbiol. 31, 955-962 (1993).

78. Espinosa, R. et al. Isolation of Neutralizing Monoclonal Antibodies to Human Astrovirus and Characterization of Virus Variants That Escape Neutralization. $J$. Virol. 93, (2019) doi: 10.1128/JVI.01465-18.

79. Molberg, O. et al. CD4+ T cells with specific reactivity against astrovirus isolated from normal human small intestine. Gastroenterology 114, 115-122 (1998) doi: $\underline{10.1016 / \mathrm{s} 0016-5085(98) 70639-0 .}$.

80. Yokoyama, C. C. et al. Adaptive immunity restricts replication of novel murine astroviruses. J. Virol. 86, 12262-12270 (2012) doi: 10.1128/JVI.02018-12.

81. Glass, R. I. et al. The changing epidemiology of astrovirus-associated gastroenteritis: a review. Arch. Virol. Suppl. 12, 287-300 (1996) doi: 10.1007/978-3-7091-6553-9 31.

82. LeBaron, C. W. et al. Viral agents of gastroenteritis. Public health importance and outbreak management. MMWR Recomm. Rep. Morb. Mortal. Wkly. Rep. Recomm. Rep. 39, 1-24 (1990).

83. Mitchell, D. K. et al. Prevalence of antibodies to astrovirus types 1 and 3 in children and adolescents in Norfolk, Virginia. Pediatr. Infect. Dis. J. 18, 249-254 (1999) doi: 10.1097/00006454-199903000-00008. 
84. Méndez-Toss, M. et al. Prevalence and genetic diversity of human astroviruses in Mexican children with symptomatic and asymptomatic infections. J. Clin.

Microbiol. 42, 151-157 (2004) doi: 10.1128/jcm.42.1.151-157.2004.

85. Maldonado, Y. et al. Population-based prevalence of symptomatic and asymptomatic astrovirus infection in rural Mayan infants. J. Infect. Dis. 178, 334339 (1998) doi: $\underline{10.1086 / 515625}$.

86. Mitchell, D. K. Astrovirus gastroenteritis. Pediatr. Infect. Dis. J. 21, 1067-1069 (2002) doi: 10.1097/00006454-200211000-00018.

87. Vu, D.-L., Cordey, S., Brito, F. \& Kaiser, L. Novel human astroviruses: Novel human diseases? J. Clin. Virol. 82, 56-63 (2016) doi: 10.1016/j.jcv.2016.07.004.

88. Quan, P.-L. et al. Astrovirus Encephalitis in Boy with X-linked Agammaglobulinemia. Emerg. Infect. Dis. 16, 918-925 (2010) doi: 10.3201/eid1606.091536.

89. Wunderli, W. et al. Astrovirus Infection in Hospitalized Infants with Severe Combined Immunodeficiency after Allogeneic Hematopoietic Stem Cell Transplantation. PLOS ONE 6, e27483 (2011) doi: 10.1371/journal.pone.0027483.

90. Cordey, S. et al. Astrovirus MLB2, a New Gastroenteric Virus Associated with Meningitis and Disseminated Infection. Emerg. Infect. Dis. 22, 846-853 (2016) doi: $10.3201 /$ eid2205.151807.

91. Sato, M. et al. Acute encephalopathy in an immunocompromised boy with astrovirus-MLB1 infection detected by next generation sequencing. J. Clin. Virol. 78, 66-70 (2016) doi: 10.1016/j.jcv.2016.03.010.

92. Brown, J. R. et al. Astrovirus VA1/HMO-C: An Increasingly Recognized Neurotropic Pathogen in Immunocompromised Patients. Clin. Infect. Dis. Off. Publ. Infect. Dis. Soc. Am. 60, 881-888 (2015) doi: 10.1093/cid/ciu940.

93. Naccache, S. N. et al. Diagnosis of Neuroinvasive Astrovirus Infection in an Immunocompromised Adult With Encephalitis by Unbiased Next-Generation Sequencing. Clin. Infect. Dis. Off. Publ. Infect. Dis. Soc. Am. 60, 919-923 (2015) doi: $10.1093 / \mathrm{cid} / \mathrm{ciu} 912$.

94. Frémond, M.-L. et al. Next-Generation Sequencing for Diagnosis and Tailored Therapy: A Case Report of Astrovirus-Associated Progressive Encephalitis. $J$. Pediatr. Infect. Dis. Soc. 4, e53-e57 (2015) doi: 10.1093/jpids/piv040. 
95. Lum, S. H. et al. An emerging opportunistic infection: fatal astrovirus (VA1/HMO-C) encephalitis in a pediatric stem cell transplant recipient. Transpl. Infect. Dis. Off. J. Transplant. Soc. (2016) doi:10.1111/tid.12607.

96. Koukou, G. et al. Human astrovirus infection associated with encephalitis in an immunocompetent child: a case report. J. Med. Case Reports 13, 341 (2019) doi: $\underline{10.1186 / \mathrm{s} 13256-019-2302-6 .}$.

97. Cortez, V. et al. Persistent Infections with Diverse Co-Circulating Astroviruses in Pediatric Oncology Patients, Memphis, Tennessee, USA. Emerg. Infect. Dis. 23, 288-290 (2017) doi: 10.3201/eid2302.161436.

98. Woode, G. N. \& Bridger, J. C. Isolation of small viruses resembling astroviruses and caliciviruses from acute enteritis of calves. J. Med. Microbiol. 11, 441-452 (1978) doi: 10.1099/00222615-11-4-441.

99. Nagai, M. et al. Full genome analysis of bovine astrovirus from fecal samples of cattle in Japan: identification of possible interspecies transmission of bovine astrovirus. Arch. Virol. 160, 2491-2501 (2015) doi: 10.1007/s00705-015-2543-7.

100. Mohamed, F. F., Mansour, S. M. G., El-Araby, I. E., Mor, S. K. \& Goyal, S. M. Molecular detection of enteric viruses from diarrheic calves in Egypt. Arch. Virol. 162, 129-137 (2017) doi: 10.1007/s00705-016-3088-0.

101. McNulty, M. S., Curran, W. L. \& McFerran, J. B. Detection of astroviruses in turkey faeces by direct electron microscopy. Vet. Rec. 106, 561 (1980) doi: 10.1136/vr.106.26.561.

102. Reynolds, D. L., Saif, Y. M. \& Theil, K. W. A survey of enteric viruses of turkey poults. Avian Dis. 31, 89-98 (1987).

103. Saif, L. J., Saif, Y. M. \& Theil, K. W. Enteric viruses in diarrheic turkey poults. Avian Dis. 29, 798-811 (1985).

104. Koci, M. D. \& Schultz-Cherry, S. Avian astroviruses. Avian Pathol. J. WVPA 31, 213-227 (2002) doi: 10.1080/03079450220136521.

105. Domanska-Blicharz, K., Seroka, A. \& Minta, Z. One-year molecular survey of astrovirus infection in turkeys in Poland. Arch. Virol. 156, 1065-1072 (2011) doi: $\underline{10.1007 / \mathrm{s} 00705-011-0958-3}$.

106. Baxendale, W. \& Mebatsion, T. The isolation and characterisation of astroviruses from chickens. Avian Pathol. J. WVPA 33, 364-370 (2004) doi: $\underline{10.1080 / 0307945042000220426 .}$. 
107. Yamaguchi, S., Imada, T. \& Kawamura, H. Characterization of a picornavirus isolated from broiler chicks. Avian Dis. 23, 571-581 (1979).

108. Bridger, J. C. Detection by electron microscopy of caliciviruses, astroviruses and rotavirus-like particles in the faeces of piglets with diarrhoea. Vet. Rec. 107, 532533 (1980).

109. Williams, F. P. Astrovirus-like, coronavirus-like, and parvovirus-like particles detected in the diarrheal stools of beagle pups. Arch. Virol. 66, 215-226 (1980) doi: $10.1007 / \mathrm{BF} 01314735$.

110. De Benedictis, P., Schultz-Cherry, S., Burnham, A. \& Cattoli, G. Astrovirus infections in humans and animals - molecular biology, genetic diversity, and interspecies transmissions. Infect. Genet. Evol. J. Mol. Epidemiol. Evol. Genet. Infect. Dis. 11, 1529-1544 (2011) doi: 10.1016/j.meegid.2011.07.024.

111. Rivera, R., Nollens, H. H., Venn-Watson, S., Gulland, F. M. D. \& Wellehan, J. F. $\mathrm{X}$. Characterization of phylogenetically diverse astroviruses of marine mammals. J. Gen. Virol. 91, 166-173 (2010) doi: 10.1099/vir.0.015222-0.

112. Hoshino, Y., Zimmer, J. F., Moise, N. S. \& Scott, F. W. Detection of astroviruses in feces of a cat with diarrhea. Brief report. Arch. Virol. 70, 373-376 (1981) doi: 10.1007/BF01320252.

113. Kang, K.-I. et al. Chicken astrovirus as an aetiological agent of runting-stunting syndrome in broiler chickens. J. Gen. Virol. 99, 512-524 (2018) doi: 10.1099/jgv.0.001025.

114. Sajewicz-Krukowska, J. \& Domanska-Blicharz, K. Nearly full-length genome sequence of a novel astrovirus isolated from chickens with 'white chicks' condition. Arch. Virol. 161, 2581-2587 (2016) doi: 10.1007/s00705-016-2940-6.

115. Sajewicz-Krukowska, J. et al. Astrovirus-induced 'white chicks' condition - field observation, virus detection and preliminary characterization. Avian Pathol. $J$. WVPA 45, 2-12 (2016) doi: 10.1080/03079457.2015.1114173.

116. Smyth, V. et al. Chicken astrovirus detected in hatchability problems associated with 'white chicks'. Vet. Rec. 173, 403-404 (2013) doi: 10.1136/vr.f6393.

117. Smyth, V. J. A Review of the Strain Diversity and Pathogenesis of Chicken Astrovirus. Viruses 9, (2017) doi: 10.3390/v9020029.

118. Schultz-Cherry, S., King, D. J. \& Koci, M. D. Inactivation of an astrovirus associated with poult enteritis mortality syndrome. Avian Dis. 45, 76-82 (2001). 
119. Schultz-Cherry, S. et al. Identifying agent(s) associated with poult enteritis mortality syndrome: importance of the thymus. Avian Dis. 44, 256-265 (2000).

120. Jindal, N., Mor, S. K. \& Goyal, S. M. Enteric viruses in turkey enteritis. VirusDisease 25, 173-185 (2014) doi: 10.1007/s13337-014-0198-8.

121. Gavier-Widén, D. et al. Investigations into shaking mink syndrome: an encephalomyelitis of unknown cause in farmed mink (Mustela vison) kits in Scandinavia. J. Vet. Diagn. Investig. Off. Publ. Am. Assoc. Vet. Lab. Diagn. Inc 16, 305-312 (2004) doi: 10.1177/104063870401600408.

122. Blomström, A.-L., Widén, F., Hammer, A.-S., Belák, S. \& Berg, M. Detection of a novel astrovirus in brain tissue of mink suffering from shaking mink syndrome by use of viral metagenomics. J. Clin. Microbiol. 48, 4392-4396 (2010) doi: 10.1128/JCM.01040-10.

123. Li, L. et al. Divergent astrovirus associated with neurologic disease in cattle. Emerg. Infect. Dis. 19, 1385-1392 (2013) doi: 10.3201/eid1909.130682.

124. Bouzalas, I. G. et al. Neurotropic astrovirus in cattle with nonsuppurative encephalitis in Europe. J. Clin. Microbiol. 52, 3318-3324 (2014) doi: 10.1128/JCM.01195-14.

125. Pfaff, F. et al. A novel astrovirus associated with encephalitis and ganglionitis in domestic sheep. Transbound. Emerg. Dis. 64, 677-682 (2017) doi: $\underline{10.1111 / \text { tbed.12623. }}$.

126. Arruda, B. et al. Porcine Astrovirus Type 3 in Central Nervous System of Swine with Polioencephalomyelitis. Emerg. Infect. Dis. 23, 2097-2100 (2017) doi: 10.3201/eid2312.170703.

127. Bosch, A., Pintó, R. M. \& Guix, S. Human Astroviruses. Clin. Microbiol. Rev. 27, 1048-1074 (2014) doi: 10.1128/CMR.00013-14.

128. Meliopoulos, V. A. et al. Detection of Antibodies against Turkey Astrovirus in Humans. PLOS ONE 9, (2014) doi: 10.1371/journal.pone.0096934.

129. Karlsson, E. A. et al. Non-Human Primates Harbor Diverse Mammalian and Avian Astroviruses Including Those Associated with Human Infections. PLOS Pathog. 11, e1005225 (2015) doi: 10.1371/journal.ppat.1005225.

130. Cubitt, W. D., Mitchell, D. K., Carter, M. J., Willcocks, M. M. \& Holzel, H. Application of electronmicroscopy, enzyme immunoassay, and RT-PCR to monitor an outbreak of astrovirus type 1 in a paediatric bone marrow transplant unit. J. Med. Virol. 57, 313-321 (1999) doi: 10.1002/(sici)10969071(199903)57:3<313::aid-jmv16>3.0.co;2-a. 
131. Herrmann, J. E. et al. Diagnosis of astrovirus gastroenteritis by antigen detection with monoclonal antibodies. J. Infect. Dis. 161, 226-229 (1990) doi: 10.1093/infdis/161.2.226.

132. Moe, C. L. et al. Detection of astrovirus in pediatric stool samples by immunoassay and RNA probe. J. Clin. Microbiol. 29, 2390-2395 (1991).

133. Noel, J. S., Lee, T. W., Kurtz, J. B., Glass, R. I. \& Monroe, S. S. Typing of human astroviruses from clinical isolates by enzyme immunoassay and nucleotide sequencing. J. Clin. Microbiol. 33, 797-801 (1995).

134. Pérot, P., Lecuit, M. \& Eloit, M. Astrovirus Diagnostics. Viruses 9, 10 (2017) doi: $\underline{10.3390 / \mathrm{v} 9010010 .}$.

135. Gu, Z. et al. Comparative Evaluation of Broad-Panel PCR Assays for the Detection of Gastrointestinal Pathogens in Pediatric Oncology Patients. J. Mol. Diagn. JMD 17, 715-721 (2015) doi: 10.1016/j.jmoldx.2015.06.003.

136. Moser, L. A. \& Schultz-Cherry, S. Pathogenesis of astrovirus infection. Viral Immunol. 18, 4-10 (2005) doi: 10.1089/vim.2005.18.4.

137. Kirkwood, C. D., Clark, R., Bogdanovic-Sakran, N. \& Bishop, R. F. A 5-year study of the prevalence and genetic diversity of human caliciviruses associated with sporadic cases of acute gastroenteritis in young children admitted to hospital in Melbourne, Australia (1998-2002). J. Med. Virol. 77, 96-101 (2005) doi: 10.1002/jmv.20419.

138. Shastri, S., Doane, A. M., Gonzales, J., Upadhyayula, U. \& Bass, D. M. Prevalence of astroviruses in a children's hospital. J. Clin. Microbiol. 36, 25712574 (1998).

139. Herrmann, J. E., Taylor, D. N., Echeverria, P. \& Blacklow, N. R. Astroviruses as a cause of gastroenteritis in children. N. Engl. J. Med. 324, 1757-1760 (1991) doi: 10.1056/NEJM199106203242501.

140. Bon, F. et al. Prevalence of group A rotavirus, human calicivirus, astrovirus, and adenovirus type 40 and 41 infections among children with acute gastroenteritis in Dijon, France. J. Clin. Microbiol. 37, 3055-3058 (1999).

141. Guerrero, M. L. et al. A prospective study of astrovirus diarrhea of infancy in Mexico City. Pediatr. Infect. Dis. J. 17, 723-727 (1998) doi: 10.1097/00006454199808000-00012. 
142. Mustafa, H., Palombo, E. A. \& Bishop, R. F. Epidemiology of astrovirus infection in young children hospitalized with acute gastroenteritis in Melbourne, Australia, over a period of four consecutive years, 1995 to 1998. J. Clin. Microbiol. 38, 1058-1062 (2000).

143. Koopmans, M. P., Bijen, M. H., Monroe, S. S. \& Vinjé, J. Age-stratified seroprevalence of neutralizing antibodies to astrovirus types 1 to 7 in humans in The Netherlands. Clin. Diagn. Lab. Immunol. 5, 33-37 (1998).

144. Kriston, S., Willcocks, M. M., Carter, M. J. \& Cubitt, W. D. Seroprevalence of astrovirus types 1 and 6 in London, determined using recombinant virus antigen. Epidemiol. Infect. 117, 159-164 (1996) doi: 10.1017/s0950268800001266.

145. Gray, J. J., Wreghitt, T. G., Cubitt, W. D. \& Elliot, P. R. An outbreak of gastroenteritis in a home for the elderly associated with astrovirus type 1 and human calicivirus. J. Med. Virol. 23, 377-381 (1987) doi: $\underline{10.1002 / j m v .1890230410 .}$.

146. Cox, G. J. et al. Etiology and outcome of diarrhea after marrow transplantation: a prospective study. Gastroenterology 107, 1398-1407 (1994) doi: $\underline{10.1016 / 0016-}$ $\underline{5085(94) 90542-8 .}$.

147. Coppo, P., Scieux, C., Ferchal, F., Clauvel, J. \& Lassoued, K. Astrovirus enteritis in a chronic lymphocytic leukemia patient treated with fludarabine monophosphate. Ann. Hematol. 79, 43-45 (2000) doi: 10.1007/s002770050008.

148. Cunliffe, N. A. \& Glass, R. I. Gastrointestinal manifestations of HIV infection. Lancet Lond. Engl. 348, 1037 (1996) doi: 10.1016/S0140-6736(05)64970-7.

149. Belliot, G., Laveran, H. \& Monroe, S. S. Outbreak of gastroenteritis in military recruits associated with serotype 3 astrovirus infection. J. Med. Virol. 51, 101106 (1997) doi: 10.1002/(sici)1096-9071(199702)51:2<101::aid-jmv3>3.0.co;2-b.

150. Pager, C. T. \& Steele, A. D. Astrovirus-associated diarrhea in South African adults. Clin. Infect. Dis. Off. Publ. Infect. Dis. Soc. Am. 35, 1452-1453 (2002) doi: $\underline{10.1086 / 344456}$.

151. Oishi, I. et al. A large outbreak of acute gastroenteritis associated with astrovirus among students and teachers in Osaka, Japan. J. Infect. Dis. 170, 439-443 (1994) doi: $\underline{10.1093 / \text { infdis/170.2.439. }}$.

152. Vu, D.-L., Bosch, A., Pintó, R. M. \& Guix, S. Epidemiology of Classic and Novel Human Astrovirus: Gastroenteritis and Beyond. Viruses 9, (2017) doi: $\underline{10.3390 / \mathrm{v} 9020033 .}$. 
153. Marshall, J. A. et al. Molecular features of astrovirus associated with a gastroenteritis outbreak in an aged-care centre. Eur. J. Clin. Microbiol. Infect. Dis. Off. Publ. Eur. Soc. Clin. Microbiol. 26, 67-71 (2007) doi: 10.1007/s10096006-0250-8.

154. Jarchow-Macdonald, A. A. et al. First report of an astrovirus type 5 gastroenteritis outbreak in a residential elderly care home identified by sequencing. J. Clin. Virol. Off. Publ. Pan Am. Soc. Clin. Virol. 73, 115-119 (2015) doi: 10.1016/j.jcv.2015.11.006.

155. Bosch, A., Guix, S. \& Pintó, R. M. Epidemiology of Human Astroviruses. in Astrovirus Research: Essential Ideas, Everyday Impacts, Future Directions (ed. Schultz-Cherry, S.) 1-18 (Springer, 2013). doi:10.1007/978-1-4614-4735-1_1.

156. Reither, K. et al. Acute childhood diarrhoea in northern Ghana: epidemiological, clinical and microbiological characteristics. BMC Infect. Dis. 7, 104 (2007) doi: $\underline{10.1186 / 1471-2334-7-104 .}$.

157. Grant, L. et al. Epidemiologic and clinical features of other enteric viruses associated with acute gastroenteritis in American Indian infants. J. Pediatr. 161, 110-115.e1 (2012) doi: 10.1016/j.jpeds.2011.12.046.

158. Resque, H. R. et al. Molecular characterization of astrovirus in stool samples from children in São Paulo, Brazil. Mem. Inst. Oswaldo Cruz 102, 969-974 (2007) doi: 10.1590/s0074-02762007000800012.

159. Palombo, E. A. \& Bishop, R. F. Annual incidence, serotype distribution, and genetic diversity of human astrovirus isolates from hospitalized children in Melbourne, Australia. J. Clin. Microbiol. 34, 1750-1753 (1996).

160. Guix, S. et al. Molecular epidemiology of astrovirus infection in Barcelona, Spain. J. Clin. Microbiol. 40, 133-139 (2002) doi: 10.1128/jem.40.1.133$\underline{139.2002 .}$.

161. Gabbay, Y. B. et al. Molecular epidemiology of astrovirus type 1 in Belém, Brazil, as an agent of infantile gastroenteritis, over a period of 18 years (19822000): identification of two possible new lineages. Virus Res. 129, 166-174 (2007) doi: 10.1016/j.virusres.2007.07.006.

162. De Grazia, S. et al. Surveillance of human astrovirus circulation in Italy 20022005: emergence of lineage 2c strains. Clin. Microbiol. Infect. Off. Publ. Eur. Soc. Clin. Microbiol. Infect. Dis. 17, 97-101 (2011) doi: 10.1111/j.14690691.2010.03207.x. 
163. Liu, M.-Q. et al. Identification of new subtype of astrovirus type 3 from an infant with diarrhea in Wuhan, China. Virology 375, 301-306 (2008) doi:

10.1016/j.virol.2008.01.032.

164. Khamrin, P. et al. Multiple astrovirus MLB1, MLB2, VA2 clades, and classic human astrovirus in children with acute gastroenteritis in Japan. J. Med. Virol. 88, 356-360 (2016) doi: 10.1002/jmv.24337.

165. Holtz, L. R. et al. Seroepidemiology of astrovirus MLB1. Clin. Vaccine Immunol. CVI 21, 908-911 (2014) doi: 10.1128/CVI.00100-14.

166. Burbelo, P. D. et al. Serological studies confirm the novel astrovirus HMOAstV$\mathrm{C}$ as a highly prevalent human infectious agent. PloS One 6, e22576 (2011) doi: 10.1371/journal.pone.0022576.

167. Björkholm, M., Celsing, F., Runarsson, G. \& Waldenström, J. Successful intravenous immunoglobulin therapy for severe and persistent astrovirus gastroenteritis after fludarabine treatment in a patient with Waldenström's macroglobulinemia. Int. J. Hematol. 62, 117-120 (1995) doi: 10.1016/09255710(95)00396-a.

168. Kurtz, J. B., Lee, T. W. \& Parsons, A. J. The action of alcohols on rotavirus, astrovirus and enterovirus. J. Hosp. Infect. 1, 321-325 (1980) doi: 10.1016/0195$\underline{6701(80) 90008-0 .}$.

169. Williams, F. Jr. Electron microscopy of stool-shed viruses: retention of characteristic morphologies after long-term storage at ultralow temperatures. Journal of Medical Virology 29, 192-5 (1989) doi: 10.1002/jmv.1890290309.

170. Morsy El-Senousy, W., Guix, S., Abid, I., Pintó, R. M. \& Bosch, A. Removal of astrovirus from water and sewage treatment plants, evaluated by a competitive reverse transcription-PCR. Appl. Environ. Microbiol. 73, 164-167 (2007) doi: 10.1128/AEM.01748-06.

171. Pintó, R. M. et al. Astrovirus detection in wastewater samples. Water Sci. Technol. J. Int. Assoc. Water Pollut. Res. 43, 73-76 (2001).

172. Abad, F. X., Pintó, R. M., Villena, C., Gajardo, R. \& Bosch, A. Astrovirus survival in drinking water. Appl. Environ. Microbiol. 63, 3119-3122 (1997).

173. Gofti-Laroche, L. et al. Are waterborne astrovirus implicated in acute digestive morbidity (E.MI.R.A. study)? J. Clin. Virol. Off. Publ. Pan Am. Soc. Clin. Virol. 27, 74-82 (2003) doi: 10.1016/s1386-6532(02)00130-0. 
174. Zhou, X. et al. An Outbreak of Gastroenteritis Associated with GII.17 NorovirusContaminated Secondary Water Supply System in Wuhan, China, 2017. Food Environ. Virol. 11, 126-137 (2019) doi: 10.1007/s12560-019-09371-7.

175. Thiery, J. P. \& Sleeman, J. P. Complex networks orchestrate epithelialmesenchymal transitions. Nat. Rev. Mol. Cell Biol. 7, 131-142 (2006) doi: $\underline{10.1038 / \mathrm{nrm} 1835}$.

176. Mani, S. A. et al. The epithelial-mesenchymal transition generates cells with properties of stem cells. Cell 133, 704-715 (2008) doi: $\underline{10.1016 / \text { j.cell.2008.03.027. }}$.

177. Morel, A.-P. et al. Generation of breast cancer stem cells through epithelialmesenchymal transition. PloS One 3, e2888 (2008) doi: 10.1371/journal.pone.0002888.

178. Gal, A. et al. Sustained TGF beta exposure suppresses Smad and non-Smad signalling in mammary epithelial cells, leading to EMT and inhibition of growth arrest and apoptosis. Oncogene 27, 1218-1230 (2008) doi: 10.1038/sj.onc. 1210741.

179. Thiery, J. P., Acloque, H., Huang, R. Y. J. \& Nieto, M. A. EpithelialMesenchymal Transitions in Development and Disease. Cell 139, 871-890 (2009) doi: $\underline{10.1016 / j . c e l 1.2009 .11 .007 .}$.

180. Ansieau, S. et al. Induction of EMT by twist proteins as a collateral effect of tumor-promoting inactivation of premature senescence. Cancer Cell 14, 79-89 (2008) doi: 10.1016/j.ccr.2008.06.005.

181. Kudo-Saito, C., Shirako, H., Takeuchi, T. \& Kawakami, Y. Cancer metastasis is accelerated through immunosuppression during Snail-induced EMT of cancer cells. Cancer Cell 15, 195-206 (2009) doi: 10.1016/j.ccr.2009.01.023.

182. Gonzalez, D. M. \& Medici, D. Signaling mechanisms of the epithelialmesenchymal transition. Sci. Signal. 7, re8 (2014) doi: $\underline{10.1126 / \text { scisignal.2005189. }}$.

183. Lamouille, S., Xu, J. \& Derynck, R. Molecular mechanisms of epithelialmesenchymal transition. Nat. Rev. Mol. Cell Biol. 15, 178-196 (2014) doi: $\underline{10.1038 / \mathrm{nrm} 3758}$.

184. Cano, A. et al. The transcription factor snail controls epithelial-mesenchymal transitions by repressing E-cadherin expression. Nat. Cell Biol. 2, 76-83 (2000) doi: $10.1038 / 35000025$. 
185. Bolós, V. et al. The transcription factor Slug represses E-cadherin expression and induces epithelial to mesenchymal transitions: a comparison with Snail and E47 repressors. J. Cell Sci. 116, 499-511 (2003) doi: 10.1242/jcs.00224.

186. Batlle, E. et al. The transcription factor snail is a repressor of E-cadherin gene expression in epithelial tumour cells. Nat. Cell Biol. 2, 84-89 (2000) doi: $\underline{10.1038 / 35000034 .}$.

187. Grooteclaes, M. L. \& Frisch, S. M. Evidence for a function of CtBP in epithelial gene regulation and anoikis. Oncogene 19, 3823-3828 (2000) doi: 10.1038/sj.onc. 1203721.

188. Comijn, J. et al. The two-handed E box binding zinc finger protein SIP1 downregulates E-cadherin and induces invasion. Mol. Cell 7, 1267-1278 (2001) doi: $10.1016 / \mathrm{s} 1097-2765(01) 00260-\mathrm{x}$.

189. Yang, J. et al. Twist, a master regulator of morphogenesis, plays an essential role in tumor metastasis. Cell 117, 927-939 (2004) doi: 10.1016/j.cell.2004.06.006.

190. Jamora, C., DasGupta, R., Kocieniewski, P. \& Fuchs, E. Links between signal transduction, transcription and adhesion in epithelial bud development. Nature 422, 317-322 (2003) doi: 10.1038/nature01458.

191. Kim, K., Lu, Z. \& Hay, E. D. Direct evidence for a role of beta-catenin/LEF-1 signaling pathway in induction of EMT. Cell Biol. Int. 26, 463-476 (2002) doi: 10.1006/cbir.2002.0901.

192. Nieto, M. A. The snail superfamily of zinc-finger transcription factors. Nat. Rev. Mol. Cell Biol. 3, 155-166 (2002) doi: 10.1038/nrm757.

193. Mauhin, V., Lutz, Y., Dennefeld, C. \& Alberga, A. Definition of the DNAbinding site repertoire for the Drosophila transcription factor SNAIL. Nucleic Acids Res. 21, 3951-3957 (1993) doi: 10.1093/nar/21.17.3951.

194. Kataoka, H. et al. A novel snail-related transcription factor Smuc regulates basic helix-loop-helix transcription factor activities via specific E-box motifs. Nucleic Acids Res. 28, 626-633 (2000) doi: 10.1093/nar/28.2.626.

195. Perez-Moreno, M. A. et al. A new role for E12/E47 in the repression of Ecadherin expression and epithelial-mesenchymal transitions. J. Biol. Chem. 276, 27424-27431 (2001) doi: 10.1074/jbc.M100827200.

196. Peinado, H., Portillo, F. \& Cano, A. Transcriptional regulation of cadherins during development and carcinogenesis. Int. J. Dev. Biol. 48, 365-375 (2004) doi: 10.1387/ijdb.041794hp. 
197. Hemavathy, K., Guru, S. C., Harris, J., Chen, J. D. \& Ip, Y. T. Human Slug is a repressor that localizes to sites of active transcription. Mol. Cell. Biol. 20, 50875095 (2000) doi: 10.1128/mcb.20.14.5087-5095.2000.

198. Peinado, H., Ballestar, E., Esteller, M. \& Cano, A. Snail mediates E-cadherin repression by the recruitment of the $\operatorname{Sin} 3 \mathrm{~A} /$ histone deacetylase 1 (HDAC1)/HDAC2 complex. Mol. Cell. Biol. 24, 306-319 (2004) doi: 10.1128/mcb.24.1.306-319.2004.

199. Tripathi, M. K. et al. Regulation of BRCA2 gene expression by the SLUG repressor protein in human breast cells. J. Biol. Chem. 280, 17163-17171 (2005) doi: $\underline{10.1074 / \text { jbc.M501375200. }}$.

200. Gnemmi, V. et al. MUC1 drives epithelial-mesenchymal transition in renal carcinoma through Wnt/ $\beta$-catenin pathway and interaction with SNAIL promoter. Cancer Lett. 346, 225-236 (2014) doi: 10.1016/j.canlet.2013.12.029.

201. Guaita, S. et al. Snail induction of epithelial to mesenchymal transition in tumor cells is accompanied by MUC1 repression and ZEB1 expression. J. Biol. Chem. 277, 39209-39216 (2002) doi: 10.1074/jbc.M206400200.

202. Jung, H., Kim, B., Moon, B. I. \& Oh, E.-S. Cytokeratin 18 is necessary for initiation of TGF- $\beta 1$-induced epithelial-mesenchymal transition in breast epithelial cells. Mol. Cell. Biochem. 423, 21-28 (2016) doi: 10.1007/s11010-0162818-7.

203. Vandewalle, C. et al. SIP1/ZEB2 induces EMT by repressing genes of different epithelial cell-cell junctions. Nucleic Acids Res. 33, 6566-6578 (2005) doi: 10.1093/nar/gki965.

204. Eger, A. et al. DeltaEF1 is a transcriptional repressor of E-cadherin and regulates epithelial plasticity in breast cancer cells. Oncogene 24, 2375-2385 (2005) doi: 10.1038/sj.onc.1208429.

205. Scott, C. L. \& Omilusik, K. D. ZEBs: Novel Players in Immune Cell Development and Function. Trends Immunol. 40, 431-446 (2019) doi: 10.1016/j.it.2019.03.001.

206. Verstappen, G. et al. Atypical Mowat-Wilson patient confirms the importance of the novel association between ZFHX1B/SIP1 and NuRD corepressor complex. Hum. Mol. Genet. 17, 1175-1183 (2008) doi: 10.1093/hmg/ddn007.

207. Postigo, A. A. \& Dean, D. C. ZEB represses transcription through interaction with the corepressor CtBP. Proc. Natl. Acad. Sci. 96, 6683-6688 (1999) doi: 10.1073/pnas.96.12.6683. 
208. van Grunsven, L. A. et al. Interaction between Smad-interacting protein-1 and the corepressor C-terminal binding protein is dispensable for transcriptional repression of E-cadherin. J. Biol. Chem. 278, 26135-26145 (2003) doi: 10.1074/jbc.M300597200.

209. Miyoshi, A. et al. Snail and SIP1 increase cancer invasion by upregulating MMP family in hepatocellular carcinoma cells. Br. J. Cancer 90, 1265-1273 (2004) doi: $\underline{10.1038 / \text { sj.bjc. } 6601685 \text {. }}$

210. Yang, F. et al. SET8 promotes epithelial-mesenchymal transition and confers TWIST dual transcriptional activities. EMBO J. 31, 110-123 (2012) doi: 10.1038/emboj.2011.364.

211. Casas, E. et al. Snail2 is an essential mediator of Twist1-induced epithelialmesenchymal transition and metastasis. Cancer Res. 71, 245-254 (2011) doi: 10.1158/0008-5472.CAN-10-2330.

212. Dave, N. et al. Functional cooperation between Snaill and twist in the regulation of ZEB1 expression during epithelial to mesenchymal transition. J. Biol. Chem. 286, 12024-12032 (2011) doi: 10.1074/jbc.M110.168625.

213. Massagué, J. How cells read TGF-beta signals. Nat. Rev. Mol. Cell Biol. 1, 169178 (2000) doi: 10.1038/35043051.

214. Hata, A. \& Chen, Y.-G. TGF- $\beta$ Signaling from Receptors to Smads. Cold Spring Harb. Perspect. Biol. 8, (2016) doi: 10.1101/cshperspect.a022061.

215. Massagué, J. TGF $\beta$ signalling in context. Nat. Rev. Mol. Cell Biol. 13, 616-630 (2012) doi: $10.1038 / \mathrm{nrm} 3434$.

216. Vincent, T. et al. A SNAIL1-SMAD3/4 transcriptional repressor complex promotes TGF-beta mediated epithelial-mesenchymal transition. Nat. Cell Biol. 11, 943-950 (2009) doi: 10.1038/ncb1905.

217. Peinado, H., Quintanilla, M. \& Cano, A. Transforming growth factor beta-1 induces snail transcription factor in epithelial cell lines: mechanisms for epithelial mesenchymal transitions. J. Biol. Chem. 278, 21113-21123 (2003) doi: $\underline{10.1074 / \mathrm{jbc} . \mathrm{M} 211304200 .}$.

218. Derynck, R. \& Zhang, Y. E. Smad-dependent and Smad-independent pathways in TGF- $\beta$ family signalling. Nature 425, 577-584 (2003) doi: 10.1038/nature02006.

219. Jaffe, A. B. \& Hall, A. Rho GTPases: biochemistry and biology. Annu. Rev. Cell Dev. Biol. 21, 247-269 (2005) doi: 10.1146/annurev.cellbio.21.020604.150721. 
220. Wang, Y.-K. et al. Bone morphogenetic protein-2-induced signaling and osteogenesis is regulated by cell shape, RhoA/ROCK, and cytoskeletal tension. Stem Cells Dev. 21, 1176-1186 (2012) doi: 10.1089/scd.2011.0293.

221. Edlund, S., Landström, M., Heldin, C.-H. \& Aspenström, P. Transforming growth factor-beta-induced mobilization of actin cytoskeleton requires signaling by small GTPases Cdc42 and RhoA. Mol. Biol. Cell 13, 902-914 (2002) doi: 10.1091/mbc.01-08-0398.

222. Bhowmick, N. A. et al. Transforming growth factor-betal mediates epithelial to mesenchymal transdifferentiation through a RhoA-dependent mechanism. Mol. Biol. Cell 12, 27-36 (2001) doi: 10.1091/mbc.12.1.27.

223. Ozdamar, B. et al. Regulation of the polarity protein Par6 by TGFbeta receptors controls epithelial cell plasticity. Science 307, 1603-1609 (2005) doi: $\underline{10.1126 / \text { science. } 1105718 \text {. }}$.

224. Ridley, A. J. Life at the leading edge. Cell 145, 1012-1022 (2011) doi: $\underline{10.1016 / \text { j.cell.2011.06.010. }}$.

225. Wilkes, M. C., Murphy, S. J., Garamszegi, N. \& Leof, E. B. Cell-Type-Specific Activation of PAK2 by Transforming Growth Factor $\beta$ Independent of Smad 2 and Smad3. Mol. Cell. Biol. 23, 8878-8889 (2003) doi: $10.1128 / \mathrm{mcb} .23 .23 .8878-$ $\underline{8889.2003 .}$.

226. Olsson, N., Piek, E., Sundström, M., ten Dijke, P. \& Nilsson, G. Transforming growth factor-beta-mediated mast cell migration depends on mitogen-activated protein kinase activity. Cell. Signal. 13, 483-490 (2001) doi: 10.1016/s0898$\underline{6568(01) 00176-0 .}$.

227. Yu, L., Hébert, M. C. \& Zhang, Y. E. TGF-beta receptor-activated p38 MAP kinase mediates Smad-independent TGF-beta responses. EMBO J. 21, 3749-3759 (2002) doi: $10.1093 / \mathrm{emboj} / \mathrm{cdf366}$.

228. Frey, R. S. \& Mulder, K. M. Involvement of extracellular signal-regulated kinase 2 and stress-activated protein kinase/Jun N-terminal kinase activation by transforming growth factor beta in the negative growth control of breast cancer cells. Cancer Res. 57, 628-633 (1997).

229. Hocevar, B. A., Brown, T. L. \& Howe, P. H. TGF-beta induces fibronectin synthesis through a c-Jun N-terminal kinase-dependent, Smad4-independent pathway. EMBO J. 18, 1345-1356 (1999) doi: 10.1093/emboj/18.5.1345.

230. Hanafusa, H. et al. Involvement of the p38 mitogen-activated protein kinase pathway in transforming growth factor-beta-induced gene expression. J. Biol. Chem. 274, 27161-27167 (1999) doi: 10.1074/jbc.274.38.27161. 
231. Bakin, A. V., Tomlinson, A. K., Bhowmick, N. A., Moses, H. L. \& Arteaga, C. L. Phosphatidylinositol 3-kinase function is required for transforming growth factor beta-mediated epithelial to mesenchymal transition and cell migration. J. Biol. Chem. 275, 36803-36810 (2000) doi: 10.1074/jbc.M005912200.

232. Lamouille, S. \& Derynck, R. Cell size and invasion in TGF-beta-induced epithelial to mesenchymal transition is regulated by activation of the mTOR pathway. J. Cell Biol. 178, 437-451 (2007) doi: 10.1083/jcb.200611146.

233. Gamell, C. et al. BMP2 induction of actin cytoskeleton reorganization and cell migration requires PI3-kinase and Cdc42 activity. J. Cell Sci. 121, 3960-3970 (2008) doi: $\underline{10.1242 / \text { jcs.031286. }}$.

234. Zhang, Y. E. Non-Smad Signaling Pathways of the TGF- $\beta$ Family. Cold Spring Harb. Perspect. Biol. 9, (2017) doi: 10.1101/cshperspect.a022129.

235. Hubbard, S. R. Structural analysis of receptor tyrosine kinases. Prog. Biophys. Mol. Biol. 71, 343-358 (1999) doi: 10.1016/s0079-6107(98)00047-9.

236. Ahmed, N. et al. Molecular pathways regulating EGF-induced epitheliomesenchymal transition in human ovarian surface epithelium. Am. J. Physiol. Cell Physiol. 290, C1532-1542 (2006) doi: 10.1152/ajpcell.00478.2005.

237. Lu, Z., Ghosh, S., Wang, Z. \& Hunter, T. Downregulation of caveolin-1 function by EGF leads to the loss of E-cadherin, increased transcriptional activity of betacatenin, and enhanced tumor cell invasion. Cancer Cell 4, 499-515 (2003) doi: $\underline{10.1016 / \mathrm{s} 1535-6108(03) 00304-0 .}$.

238. Lo, H.-W. et al. Epidermal growth factor receptor cooperates with signal transducer and activator of transcription 3 to induce epithelial-mesenchymal transition in cancer cells via up-regulation of TWIST gene expression. Cancer Res. 67, 9066-9076 (2007) doi: 10.1158/0008-5472.CAN-07-0575.

239. Strutz, F. et al. Role of basic fibroblast growth factor- 2 in epithelial-mesenchymal transformation. Kidney Int. 61, 1714-1728 (2002) doi: 10.1046/j.15231755.2002.00333.x.

240. Lee, J. G. \& Kay, E. P. Cross-talk among Rho GTPases acting downstream of PI 3-kinase induces mesenchymal transformation of corneal endothelial cells mediated by FGF-2. Invest. Ophthalmol. Vis. Sci. 47, 2358-2368 (2006) doi: 10.1167/iovs.05-1490. 
241. Kim, H.-J. et al. Constitutively active type I insulin-like growth factor receptor causes transformation and xenograft growth of immortalized mammary epithelial cells and is accompanied by an epithelial-to-mesenchymal transition mediated by NF-kappaB and snail. Mol. Cell. Biol. 27, 3165-3175 (2007) doi: 10.1128/MCB.01315-06.

242. Graham, T. R. et al. Insulin-like growth factor-I-dependent up-regulation of ZEB1 drives epithelial-to-mesenchymal transition in human prostate cancer cells. Cancer Res. 68, 2479-2488 (2008) doi: 10.1158/0008-5472.CAN-07-2559.

243. Irie, H. Y. et al. Distinct roles of Akt1 and Akt2 in regulating cell migration and epithelial-mesenchymal transition. J. Cell Biol. 171, 1023-1034 (2005) doi: 10.1083/jcb.200505087.

244. Wanami, L. S., Chen, H.-Y., Peiró, S., García de Herreros, A. \& Bachelder, R. E. Vascular endothelial growth factor-A stimulates Snail expression in breast tumor cells: implications for tumor progression. Exp. Cell Res. 314, 2448-2453 (2008) doi: $10.1016 /$ j.yexcr.2008.05.004.

245. Park, J. A., Kim, D. Y., Kim, Y.-M., Lee, I.-K. \& Kwon, Y.-G. Endothelial Snail Regulates Capillary Branching Morphogenesis via Vascular Endothelial Growth Factor Receptor 3 Expression. PLOS Genet. 11, e1005324 (2015) doi: 10.1371/journal.pgen.1005324.

246. Peinado, H. et al. Snail and E47 repressors of E-cadherin induce distinct invasive and angiogenic properties in vivo. J. Cell Sci. 117, 2827-2839 (2004) doi: $10.1242 /$ jes. 01145 .

247. Yang, X., Chrisman, H. \& Weijer, C. J. PDGF signalling controls the migration of mesoderm cells during chick gastrulation by regulating N-cadherin expression. Dev. Camb. Engl. 135, 3521-3530 (2008) doi: 10.1242/dev.023416.

248. Das, V., Bhattacharya, S., Chikkaputtaiah, C., Hazra, S. \& Pal, M. The basics of epithelial-mesenchymal transition (EMT): A study from a structure, dynamics, and functional perspective. J. Cell. Physiol. 234, 14535-14555 (2019) doi:

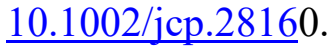

249. Niehrs, C. The complex world of WNT receptor signalling. Nat. Rev. Mol. Cell Biol. 13, 767-779 (2012) doi: 10.1038/nrm3470.

250. MacDonald, B. T., Tamai, K. \& He, X. Wnt/ß-catenin signaling: components, mechanisms, and diseases. Dev. Cell 17, 9-26 (2009) doi: 10.1016/j.devcel.2009.06.016.

251. Clevers, H. Wnt/beta-catenin signaling in development and disease. Cell 127, 469-480 (2006) doi: 10.1016/j.cell.2006.10.018. 
252. Zhou, B. P. et al. Dual regulation of Snail by GSK-3beta-mediated phosphorylation in control of epithelial-mesenchymal transition. Nat. Cell Biol. 6, 931-940 (2004) doi: $10.1038 / \mathrm{ncb} 1173$.

253. García-Castro, M. I., Marcelle, C. \& Bronner-Fraser, M. Ectodermal Wnt function as a neural crest inducer. Science 297, 848-851 (2002).

254. Liu, P. et al. Requirement for Wnt3 in vertebrate axis formation. Nat. Genet. 22, 361-365 (1999) doi: 10.1038/11932.

255. Verras, M. \& Sun, Z. Roles and regulation of Wnt signaling and beta-catenin in prostate cancer. Cancer Lett. 237, 22-32 (2006) doi: 10.1016/j.canlet.2005.06.004.

256. Mukherjee, N. \& Panda, C. K. Wnt/ß-Catenin Signaling Pathway as Chemotherapeutic Target in Breast Cancer: An Update on Pros and Cons. Clin. Breast Cancer (2020) doi:10.1016/j.clbc.2020.04.004.

257. Brabletz, T. et al. Variable beta-catenin expression in colorectal cancers indicates tumor progression driven by the tumor environment. Proc. Natl. Acad. Sci. U. S. A. 98, 10356-10361 (2001) doi: 10.1073/pnas.171610498.

258. Wei, Y. et al. Activation of $\beta$-catenin in epithelial and mesenchymal hepatoblastomas. Oncogene 19, 498-504 (2000) doi: 10.1038/sj.onc.1203356.

259. Bray, S. J. Notch signalling: a simple pathway becomes complex. Nat. Rev. Mol. Cell Biol. 7, 678-689 (2006) doi: 10.1038/nrm2009.

260. Sahlgren, C., Gustafsson, M. V., Jin, S., Poellinger, L. \& Lendahl, U. Notch signaling mediates hypoxia-induced tumor cell migration and invasion. Proc. Natl. Acad. Sci. U. S. A. 105, 6392-6397 (2008) doi: 10.1073/pnas.0802047105.

261. Leong, K. G. et al. Jagged1-mediated Notch activation induces epithelial-tomesenchymal transition through Slug-induced repression of E-cadherin. J. Exp. Med. 204, 2935-2948 (2007) doi: 10.1084/jem.20071082.

262. Niessen, K. et al. Slug is a direct Notch target required for initiation of cardiac cushion cellularization. J. Cell Biol. 182, 315-325 (2008) doi: 10.1083/jcb.200710067.

263. Xie, M. et al. Activation of Notch-1 enhances epithelial-mesenchymal transition in gefitinib-acquired resistant lung cancer cells. J. Cell. Biochem. 113, 1501-1513 (2012) doi: 10.1002/jcb.24019.

264. Katoh, Y. \& Katoh, M. Hedgehog signaling, epithelial-to-mesenchymal transition and miRNA (review). Int. J. Mol. Med. 22, 271-275 (2008). 
265. Li, X. et al. Snail induction is an early response to Glil that determines the efficiency of epithelial transformation. Oncogene 25, 609-621 (2006) doi: 10.1038/sj.onc.1209077.

266. Yoo, Y. A., Kang, M. H., Kim, J. S. \& Oh, S. C. Sonic hedgehog signaling promotes motility and invasiveness of gastric cancer cells through TGF-betamediated activation of the ALK5-Smad 3 pathway. Carcinogenesis 29, 480-490 (2008) doi: 10.1093/carcin/bgm281.

267. Monsoro-Burq, A.-H. Sclerotome development and morphogenesis: when experimental embryology meets genetics. Int. J. Dev. Biol. 49, 301-308 (2005) doi: $10.1387 / \mathrm{ijdb} .041953 \mathrm{am}$.

268. Teillet, M. et al. Sonic hedgehog is required for survival of both myogenic and chondrogenic somitic lineages. Dev. Camb. Engl. 125, 2019-2030 (1998).

269. Abba, M. L., Patil, N., Leupold, J. H. \& Allgayer, H. MicroRNA Regulation of Epithelial to Mesenchymal Transition. J. Clin. Med. 5, (2016) doi: 10.3390/jcm5010008.

270. Musavi Shenas, M. H. et al. MicroRNAs and signaling networks involved in epithelial-mesenchymal transition. J. Cell. Physiol. 234, 5775-5785 (2019) doi:

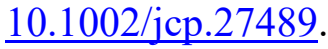

271. Zhang, J. \& Ma, L. MicroRNA control of epithelial-mesenchymal transition and metastasis. Cancer Metastasis Rev. 31, 653-662 (2012) doi: 10.1007/s10555-0129368-6.

272. Alidadiani, N., Ghaderi, S., Dilaver, N., Bakhshamin, S. \& Bayat, M. Epithelial mesenchymal transition Transcription Factor (TF): The structure, function and microRNA feedback loop. Gene 674, 115-120 (2018) doi: 10.1016/j.gene.2018.06.049.

273. Gregory, P. A. et al. The miR-200 family and miR-205 regulate epithelial to mesenchymal transition by targeting ZEB1 and SIP1. Nat. Cell Biol. 10, 593-601 (2008) doi: $10.1038 /$ ncb1722.

274. Park, S.-M., Gaur, A. B., Lengyel, E. \& Peter, M. E. The miR-200 family determines the epithelial phenotype of cancer cells by targeting the E-cadherin repressors ZEB1 and ZEB2. Genes Dev. 22, 894-907 (2008) doi: 10.1101/gad.1640608.

275. Bracken, C. P. et al. A double-negative feedback loop between ZEB1-SIP1 and the microRNA-200 family regulates epithelial-mesenchymal transition. Cancer Res. 68, 7846-7854 (2008) doi: 10.1158/0008-5472.CAN-08-1942. 
276. Korpal, M., Lee, E. S., Hu, G. \& Kang, Y. The miR-200 family inhibits epithelialmesenchymal transition and cancer cell migration by direct targeting of Ecadherin transcriptional repressors ZEB1 and ZEB2. J. Biol. Chem. 283, 1491014914 (2008) doi: 10.1074/jbc.C800074200.

277. Kumarswamy, R. et al. MicroRNA-30a inhibits epithelial-to-mesenchymal transition by targeting Snail and is downregulated in non-small cell lung cancer. Int. J. Cancer 130, 2044-2053 (2012) doi: 10.1002/ijc.26218.

278. Ding, X., Park, S. I., McCauley, L. K. \& Wang, C.-Y. Signaling between transforming growth factor $\beta$ (TGF- $\beta$ ) and transcription factor SNAI2 represses expression of microRNA miR-203 to promote epithelial-mesenchymal transition and tumor metastasis. J. Biol. Chem. 288, 10241-10253 (2013) doi: $\underline{10.1074 / \mathrm{jbc} . \mathrm{M} 112.443655 .}$.

279. Zhang, Z. et al. Epigenetic Silencing of miR-203 Upregulates SNAI2 and Contributes to the Invasiveness of Malignant Breast Cancer Cells. Genes Cancer 2, 782-791 (2011) doi: 10.1177/1947601911429743.

280. Siemens, H. et al. miR-34 and SNAIL form a double-negative feedback loop to regulate epithelial-mesenchymal transitions. Cell Cycle 10, 4256-4271 (2011) doi: $\underline{10.4161 / \mathrm{cc} \cdot 10.24 .18552}$.

281. Wang, H. et al. TGF- $\beta 1$-induced epithelial-mesenchymal transition in lung cancer cells involves upregulation of miR-9 and downregulation of its target, E-cadherin. Cell. Mol. Biol. Lett. 22, 22 (2017) doi: 10.1186/s11658-017-0053-1.

282. Sui, X., Jiao, Y.-N., Yang, L.-H. \& Liu, J. MiR-9 accelerates epithelialmesenchymal transition of ovarian cancer cells via inhibiting e-cadherin. Eur. Rev. Med. Pharmacol. Sci. 23, 209-216 (2019) doi: 10.26355/eurrev_201908_18649.

283. $\mathrm{Xu}, \mathrm{X}$.-Z. et al. MiR-9 promotes synovial sarcoma cell migration and invasion by directly targeting CDH1. Int. J. Biochem. Cell Biol. 112, 61-71 (2019) doi: $\underline{10.1016 / \text { j.biocel.2019.04.001. }}$.

284. Huang, Q. et al. The microRNAs miR-373 and miR-520c promote tumour invasion and metastasis. Nat. Cell Biol. 10, 202-210 (2008) doi: $\underline{10.1038 / \mathrm{ncb} 1681 .}$.

285. Zeisberg, M. \& Neilson, E. G. Biomarkers for epithelial-mesenchymal transitions. J. Clin. Invest. 119, 1429-1437 (2009) doi: 10.1172/JCI36183.

286. Hay, E. D. Role of cell-matrix contacts in cell migration and epithelialmesenchymal transformation. Cell Differ. Dev. 32, 367-375 (1990) doi: $\underline{10.1016 / 0922-3371(90) 90052-\mathrm{x}}$. 
287. Thomas, P., Brickman, J., Pöpperl, H., Krumlauf, R. \& Beddington, R. S. Axis Duplication and Anterior Identity in the Mouse Embryo. Cold Spring Harbor symposia on quantitative biology vol. 62 https://pubmed.ncbi.nlm.nih.gov/9598343/ (1997).

288. Pöpperl, H. et al. Misexpression of Cwnt8C in the Mouse Induces an Ectopic Embryonic Axis and Causes a Truncation of the Anterior Neuroectoderm. Development (Cambridge, England) vol. 124 https://pubmed.ncbi.nlm.nih.gov/9247341/ (1997).

289. Ciruna, B. \& Rossant, J. FGF Signaling Regulates Mesoderm Cell Fate Specification and Morphogenetic Movement at the Primitive Streak. Dev. Cell 1, 37-49 (2001) doi: 10.1016/s1534-5807(01)00017-x.

290. Sela-Donenfeld, D. \& Kalcheim, C. Localized BMP4-Noggin Interactions Generate the Dynamic Patterning of Noggin Expression in Somites. Dev. Biol. 246, 311-328 (2002) doi: 10.1006/dbio.2002.0672.

291. Burstyn-Cohen, T. Canonical Wnt activity regulates trunk neural crest delamination linking BMP/noggin signaling with G1/S transition. Development 131, 5327-5339 (2004) doi: 10.1242/dev.01424.

292. Kalluri, R. \& Weinberg, R. A. The basics of epithelial-mesenchymal transition. $J$. Clin. Invest. 119, 1420-1428 (2009) doi: 10.1172/JCI39104.

293. Zeisberg, E. M. et al. Endothelial-to-mesenchymal transition contributes to cardiac fibrosis. Nat. Med. 13, 952-961 (2007) doi: 10.1038/nm1613.

294. Zeisberg, M. \& Kalluri, R. Cellular Mechanisms of Tissue Fibrosis. 1. Common and organ-specific mechanisms associated with tissue fibrosis. Am. J. Physiol. Cell Physiol. 304, C216-C225 (2013) doi: 10.1152/ajpcell.00328.2012.

295. Zeisberg, M. et al. BMP-7 counteracts TGF- $\beta 1$-induced epithelial-tomesenchymal transition and reverses chronic renal injury. Nat. Med. 9, 964-968 (2003) doi: $\underline{10.1038 / \mathrm{nm} 888}$.

296. Luo, D. D., Phillips, A. \& Fraser, D. Bone Morphogenetic Protein-7 Inhibits Proximal Tubular Epithelial Cell Smad3 Signaling via Increased SnoN Expression. Am. J. Pathol. 176, 1139-1147 (2010) doi: 10.2353/ajpath.2010.090459.

297. Morrissey, J. et al. Bone Morphogenetic protein-7 Improves Renal Fibrosis and Accelerates the Return of Renal Function. Journal of the American Society of Nephrology: JASN vol. 13 Suppl 1 https://pubmed.ncbi.nlm.nih.gov/11792757/ (2002). 
298. Zeisberg, M. et al. Bone morphogenic protein-7 inhibits progression of chronic renal fibrosis associated with two genetic mouse models. Am. J. Physiol.-Ren. Physiol. 285, F1060-F1067 (2003) doi: 10.1152/ajprenal.00191.2002.

299. Kalluri, R. EMT: When epithelial cells decide to become mesenchymal-like cells. J. Clin. Invest. 119, 1417-1419 (2009) doi: 10.1172/JCI39675.

300. Aiello, N. M. \& Kang, Y. Context-dependent EMT programs in cancer metastasis. J. Exp. Med. 216, 1016-1026 (2019) doi: 10.1084/jem.20181827.

301. Tsai, J. H., Donaher, J. L., Murphy, D. A., Chau, S. \& Yang, J. Spatiotemporal regulation of epithelial-mesenchymal transition is essential for squamous cell carcinoma metastasis. Cancer Cell 22, 725-736 (2012) doi: $\underline{10.1016 / j . c c r .2012 .09 .02}$

302. Khoo, B. L. et al. Short-term expansion of breast circulating cancer cells predicts response to anti-cancer therapy. Oncotarget 6, 15578-15593 (2015) doi: 10.18632/oncotarget.3903.

303. Yu, M. et al. Circulating Breast Tumor Cells Exhibit Dynamic Changes in Epithelial and Mesenchymal Composition. 339, 7 (2013) doi: 10.1126/science. 1228522 .

304. Acloque, H., Adams, M. S., Fishwick, K., Bronner-Fraser, M. \& Nieto, M. A. Epithelial-mesenchymal transitions: the importance of changing cell state in development and disease. J. Clin. Invest. 119, 1438-1449 (2009) doi: 10.1172/JCI38019.

305. Yang, A. D. et al. Chronic oxaliplatin resistance induces epithelial-tomesenchymal transition in colorectal cancer cell lines. Clin. Cancer Res. Off. J. Am. Assoc. Cancer Res. 12, 4147-4153 (2006) doi: 10.1158/1078-0432.CCR-06$\underline{0038}$.

306. Kajiyama, H. et al. Chemoresistance to paclitaxel induces epithelial-mesenchymal transition and enhances metastatic potential for epithelial ovarian carcinoma cells. Int. J. Oncol. 31, 277-283 (2007).

307. Liu, Y., El-Naggar, S., Darling, D. S., Higashi, Y. \& Dean, D. C. Zeb1 links epithelial-mesenchymal transition and cellular senescence. Dev. Camb. Engl. 135, 579-588 (2008) doi: 10.1242/dev.007047.

308. Gupta, P. B., Chaffer, C. L. \& Weinberg, R. A. Cancer stem cells: mirage or reality? Nat. Med. 15, 1010-1012 (2009) doi: 10.1038/nm0909-1010. 
309. Li, W. et al. Unraveling the roles of CD44/CD24 and ALDH1 as cancer stem cell markers in tumorigenesis and metastasis. Sci. Rep. 7, 13856 (2017) doi: 10.1038/s41598-017-14364-2.

310. Saitoh, M. Involvement of partial EMT in cancer progression. J. Biochem. (Tokyo) 164, 257-264 (2018) doi: 10.1093/jb/mvy047.

311. Shibue, T. \& Weinberg, R. A. EMT, CSCs, and drug resistance: the mechanistic link and clinical implications. Nat. Rev. Clin. Oncol. 14, 611-629 (2017) doi: $\underline{10.1038 / \text { nrclinonc.2017.44. }}$.

312. Nieto, M. A., Huang, R. Y.-J., Jackson, R. A. \& Thiery, J. P. EMT: 2016. Cell 166, 21-45 (2016) doi: 10.1016/j.cell.2016.06.028.

313. Grigore, A. D., Jolly, M. K., Jia, D., Farach-Carson, M. C. \& Levine, H. Tumor Budding: The Name is EMT. Partial EMT. J. Clin. Med. 5, (2016) doi: 10.3390/jcm5050051.

314. Arnoux, V., Nassour, M., L'Helgoualc'h, A., Hipskind, R. A. \& Savagner, P. Erk5 Controls Slug Expression and Keratinocyte Activation during Wound Healing. Mol. Biol. Cell 19, $4738-4749$ (2008) doi: 10.1091/mbc.e07-10-1078.

315. Grande, M. T. et al. Snaill-induced partial epithelial-to-mesenchymal transition drives renal fibrosis in mice and can be targeted to reverse established disease. Nat. Med. 21, 989-997 (2015) doi: 10.1038/nm.3901.

316. Blanco, M. J. et al. Snailla and Snail $1 \mathrm{~b}$ cooperate in the anterior migration of the axial mesendoderm in the zebrafish embryo. Dev. Camb. Engl. 134, 4073-4081 (2007) doi: $10.1242 / \mathrm{dev} .006858$.

317. Friedl, P., Locker, J., Sahai, E. \& Segall, J. E. Classifying collective cancer cell invasion. Nat. Cell Biol. 14, 777-783 (2012) doi: 10.1038/ncb2548.

318. Clark, A. G. \& Vignjevic, D. M. Modes of cancer cell invasion and the role of the microenvironment. Curr. Opin. Cell Biol. 36, 13-22 (2015) doi: 10.1016/j.ceb.2015.06.004.

319. Shin Kim, S., Yeom, S., Kwak, J., Ahn, H.-J. \& Lib Jang, K. Hepatitis B virus X protein induces epithelial-mesenchymal transition by repressing E-cadherin expression via upregulation of E12/E47. J. Gen. Virol. 97, 134-143 (2016) doi: 10.1099/jgv.0.000324.

320. Bose, S. K., Meyer, K., Di Bisceglie, A. M., Ray, R. B. \& Ray, R. Hepatitis C virus induces epithelial-mesenchymal transition in primary human hepatocytes. $J$. Virol. 86, 13621-13628 (2012) doi: 10.1128/JVI.02016-12. 
321. Chamulitrat, W., Sattayakhom, A., Herold-Mende, C., Herold-Mended, C. \& Stremmel, W. Human papillomavirus 16 E6/E7-immortalized human gingival keratinocytes with epithelial mesenchymal transition acquire increased expression of cIAP-1, Bclx and p27(Kip1). Exp. Dermatol. 18, 1067-1069 (2009) doi: 10.1111/j.1600-0625.2009.00888.x.

322. Kim, S.-M. et al. Epstein-Barr virus-encoded latent membrane protein 1 induces epithelial to mesenchymal transition by inducing V-set Ig domain containing 4 (VSIG4) expression via NF-kB in renal tubular epithelial HK-2 cells. Biochem. Biophys. Res. Commun. 492, 316-322 (2017) doi: 10.1016/j.bbrc.2017.08.116.

323. Gaur, N., Tikla, T. \& Kaul, R. Kaposi sarcoma-associated herpes virus (KSHV) latent protein LANA modulates cellular genes associated with epithelial-tomesenchymal transition. Arch. Virol. 164, 91-104 (2019) doi: 10.1007/s00705018-4060-y.

324. Shimamura, M., Murphy-Ullrich, J. E. \& Britt, W. J. Human cytomegalovirus induces TGF- $\beta 1$ activation in renal tubular epithelial cells after epithelial-tomesenchymal transition. PLoS Pathog. 6, e1001170 (2010) doi: $\underline{10.1371 / \text { journal.ppat.1001170. }}$.

325. Teo, W. H., Chen, H.-P., Huang, J. C. \& Chan, Y.-J. Human cytomegalovirus infection enhances cell proliferation, migration and upregulation of EMT markers in colorectal cancer-derived stem cell-like cells. Int. J. Oncol. 51, 1415-1426 (2017) doi: 10.3892/ijo.2017.4135.

326. Chen, X., Bode, A. M., Dong, Z. \& Cao, Y. The epithelial-mesenchymal transition (EMT) is regulated by oncoviruses in cancer. FASEB J. Off. Publ. Fed. Am. Soc. Exp. Biol. 30, 3001-3010 (2016) doi: 10.1096/fj.201600388R.

327. McLaughlin-Drubin, M. E. \& Munger, K. Viruses associated with human cancer. Biochim. Biophys. Acta 1782, 127-150 (2008) doi: 10.1016/j.bbadis.2007.12.005.

328. Zha, Y., Yao, Q., Liu, J.-S., Wang, Y.-Y. \& Sun, W.-M. Hepatitis B virus X protein promotes epithelial-mesenchymal transition and metastasis in hepatocellular carcinoma cell line HCCLM3 by targeting HMGA2. Oncol. Lett. 16, 5709-5714 (2018) doi: 10.3892/ol.2018.9359.

329. Jin, Y. et al. Hepatitis B virus x protein induces epithelial-mesenchymal transition of hepatocellular carcinoma cells by regulating long non-coding RNA. Virol. $J$. 14, (2017) doi: 10.1186/s12985-017-0903-5.

330. Li, M. et al. Hepatitis B virus X protein promotes renal epithelial-mesenchymal transition in human renal proximal tubule epithelial cells through the activation of NF-kB. Int. J. Mol. Med. 38, 513-520 (2016) doi: 10.3892/ijmm.2016.2637. 
331. $\mathrm{Hu}, \mathrm{B}$. et al. Hepatitis $\mathrm{C}$ virus NS4B protein induces epithelial-mesenchymal transition by upregulation of Snail. Virol. J. 14, (2017) doi: 10.1186/s12985-017$\underline{0737-1 .}$.

332. Tiwari, I., Yoon, M.-H., Park, B.-J. \& Jang, K. L. Hepatitis C virus core protein induces epithelial-mesenchymal transition in human hepatocytes by upregulating E12/E47 levels. Cancer Lett. 362, 131-138 (2015) doi: 10.1016/j.canlet.2015.03.032.

333. Zhou, J.-J. et al. Hepatitis C virus core protein increases Snail expression and induces epithelial-mesenchymal transition through the signal transducer and activator of transcription 3 pathway in hepatoma cells. Hepatol. Res. 47, 574-583 (2017) doi: $\underline{10.1111 / \text { hepr.12771. }}$

334. Liu, D. et al. Downregulation of miRNA-30c and miR-203a is associated with hepatitis $\mathrm{C}$ virus core protein-induced epithelial-mesenchymal transition in normal hepatocytes and hepatocellular carcinoma cells. Biochem. Biophys. Res. Commun. 464, 1215-1221 (2015) doi: 10.1016/j.bbrc.2015.07.107.

335. Ranieri, D., Belleudi, F., Magenta, A. \& Torrisi, M. R. HPV16 E5 expression induces switching from FGFR2b to FGFR2c and epithelial-mesenchymal transition. Int. J. Cancer 137, 61-72 (2015) doi: 10.1002/ijc.29373.

336. Jung, Y.-S., Kato, I. \& Kim, H.-R. C. A novel function of HPV16-E6/E7 in epithelial-mesenchymal transition. Biochem. Biophys. Res. Commun. 435, 339344 (2013) doi: $\underline{10.1016 / j . b b r c .2013 .04 .060}$.

337. Gaur, N., Gandhi, J., Robertson, E. S., Verma, S. C. \& Kaul, R. Epstein-Barr virus latent antigens EBNA3C and EBNA1 modulate epithelial to mesenchymal transition of cancer cells associated with tumor metastasis. Tumour Biol. J. Int. Soc. Oncodevelopmental Biol. Med. 36, 3051-3060 (2015) doi: 10.1007/s13277014-2941-6.

338. Morris, M. A. et al. The EBV-Encoded Oncoprotein, LMP1, Induces an Epithelial-to-Mesenchymal Transition (EMT) via Its CTAR1 Domain through Integrin-Mediated ERK-MAPK Signalling. Cancers 10, (2018) doi: $\underline{10.3390 / \text { cancers } 10050130 \text {. }}$.

339. Wasil, L. R. \& Shair, K. H. Y. Epstein-Barr virus LMP1 induces focal adhesions and epithelial cell migration through effects on integrin- $\alpha 5$ and N-cadherin. Oncogenesis 4, e171 (2015) doi: 10.1038/oncsis.2015.31.

340. Minor, D. M. \& Proud, D. Role of human rhinovirus in triggering human airway epithelial-mesenchymal transition. Respir. Res. 18, 110 (2017) doi: 10.1186/s12931-017-0595-9. 
341. Xia, L., Dai, L., Yu, Q. \& Yang, Q. Persistent TGEV infection enhances ETEC $\mathrm{K} 88$ adhesion by promoting epithelial-mesenchymal transition in intestinal epithelial cells. J. Virol. (2017) doi:10.1128/JVI.01256-17.

342. Hargest, V., Sharp, B., Livingston, B., Cortez, V. \& Schultz-Cherry, S. Astrovirus Replication Is Inhibited by Nitazoxanide In Vitro and In Vivo. J. Virol. 94, (2020) doi: $\underline{10.1128 / J V I .01706-19}$.

343. Marvin, S., Meliopoulos, V. \& Schultz-Cherry, S. Human Astrovirus Propagation, Purification and Quantification. BIO-Protoc. 4, (2014).

344. Abe, M. et al. An assay for transforming growth factor-beta using cells transfected with a plasminogen activator inhibitor-1 promoter-luciferase construct. Anal. Biochem. 216, 276-284 (1994) doi: 10.1006/abio.1994.1042.

345. Weber, F., Wagner, V., Rasmussen, S. B., Hartmann, R. \& Paludan, S. R. DoubleStranded RNA Is Produced by Positive-Strand RNA Viruses and DNA Viruses but Not in Detectable Amounts by Negative-Strand RNA Viruses. J. Virol. 80, 5059-5064 (2006) doi: 10.1128/JVI.80.10.5059-5064.2006.

346. Johnson, C., Hargest, V., Cortez, V., Meliopoulos, V. A. \& Schultz-Cherry, S. Astrovirus Pathogenesis. Viruses 9, (2017) doi: 10.3390/v9010022.

347. Ulluwishewa, D. et al. Regulation of tight junction permeability by intestinal bacteria and dietary components. J. Nutr. 141, 769-776 (2011) doi: $\underline{10.3945 / \text { jn. } 110.135657 .}$.

348. Odenwald, M. A. \& Turner, J. R. Intestinal permeability defects: is it time to treat? Clin. Gastroenterol. Hepatol. Off. Clin. Pract. J. Am. Gastroenterol. Assoc. 11, 1075-1083 (2013) doi: 10.1016/j.cgh.2013.07.001.

349. Moser, L. A., Carter, M. \& Schultz-Cherry, S. Astrovirus increases epithelial barrier permeability independently of viral replication. J. Virol. 81, 11937-11945 (2007) doi: 10.1128/JVI.00942-07.

350. Meliopoulos, V. A. et al. Oral Administration of Astrovirus Capsid Protein Is Sufficient To Induce Acute Diarrhea In Vivo. mBio 7, (2016) doi: $\underline{10.1128 / \mathrm{mBio} .01494-16}$.

351. Gonzalez, D. M. \& Medici, D. Signaling mechanisms of the epithelialmesenchymal transition. Sci. Signal. 7, re8 (2014) doi: 10.1126/scisignal.2005189.

352. Moustakas, A. \& Heldin, C.-H. Signaling networks guiding epithelialmesenchymal transitions during embryogenesis and cancer progression. Cancer Sci. 98, 1512-1520 (2007) doi: 10.1111/j.1349-7006.2007.00550.x. 
353. Tsubakihara, Y. \& Moustakas, A. Epithelial-Mesenchymal Transition and Metastasis under the Control of Transforming Growth Factor $\beta$. Int. J. Mol. Sci. 19, (2018) doi: 10.3390/ijms19113672.

354. Shin Kim, S., Yeom, S., Kwak, J., Ahn, H.-J. \& Lib Jang, K. Hepatitis B virus X protein induces epithelial-mesenchymal transition by repressing E-cadherin expression via upregulation of E12/E47. J. Gen. Virol. 97, 134-143 (2016) doi: 10.1099/jgv.0.000324.

355. Bose, S. K., Meyer, K., Di Bisceglie, A. M., Ray, R. B. \& Ray, R. Hepatitis C virus induces epithelial-mesenchymal transition in primary human hepatocytes. $J$. Virol. 86, 13621-13628 (2012) doi: 10.1128/JVI.02016-12.

356. Chamulitrat, W., Sattayakhom, A., Herold-Mende, C., Herold-Mended, C. \& Stremmel, W. Human papillomavirus 16 E6/E7-immortalized human gingival keratinocytes with epithelial mesenchymal transition acquire increased expression of cIAP-1, Bclx and p27(Kip1). Exp. Dermatol. 18, 1067-1069 (2009) doi: 10.1111/j.1600-0625.2009.00888.x.

357. Horikawa, T. et al. Twist and epithelial-mesenchymal transition are induced by the EBV oncoprotein latent membrane protein 1 and are associated with metastatic nasopharyngeal carcinoma. Cancer Res. 67, 1970-1978 (2007) doi: 10.1158/0008-5472.CAN-06-3933.

358. Shimamura, M., Murphy-Ullrich, J. E. \& Britt, W. J. Human cytomegalovirus induces TGF- $\beta 1$ activation in renal tubular epithelial cells after epithelial-tomesenchymal transition. PLoS Pathog. 6, e1001170 (2010) doi: 10.1371/journal.ppat.1001170.

359. Teo, W. H., Chen, H.-P., Huang, J. C. \& Chan, Y.-J. Human cytomegalovirus infection enhances cell proliferation, migration and upregulation of EMT markers in colorectal cancer-derived stem cell-like cells. Int. J. Oncol. 51, 1415-1426 (2017) doi: 10.3892/ijo.2017.4135.

360. Minor, D. M. \& Proud, D. Role of human rhinovirus in triggering human airway epithelial-mesenchymal transition. Respir. Res. 18, 110 (2017) doi: 10.1186/s12931-017-0595-9.

361. Faris, A. N. et al. Rhinovirus Delays Cell Repolarization in a Model of Injured/Regenerating Human Airway Epithelium. Am. J. Respir. Cell Mol. Biol. 55, 487-499 (2016) doi: 10.1165/rcmb.2015-0243OC.

362. van Roy, F. \& Berx, G. The cell-cell adhesion molecule E-cadherin. Cell. Mol. Life Sci. 65, 3756-3788 (2008) doi: 10.1007/s00018-008-8281-1. 
363. Gumbiner, B., Stevenson, B. \& Grimaldi, A. The role of the cell adhesion molecule uvomorulin in the formation and maintenance of the epithelial junctional complex. J. Cell Biol. 107, 1575-1587 (1988) doi: 10.1083/jcb.107.4.1575.

364. Lewis, J. E. et al. Cross-talk between adherens junctions and desmosomes depends on plakoglobin. J. Cell Biol. 136, 919-934 (1997) doi: $\underline{10.1083 / \mathrm{jcb} .136 .4 .919 .}$.

365. Moreno-Bueno, G., Portillo, F. \& Cano, A. Transcriptional regulation of cell polarity in EMT and cancer. Oncogene 27, 6958-6969 (2008) doi: 10.1038/onc.2008.346.

366. Thiery, J. P. Epithelial-mesenchymal transitions in development and pathologies. Curr. Opin. Cell Biol. 15, 740-746 (2003) doi: 10.1016/j.ceb.2003.10.006.

367. Pelaseyed, T. \& Bretscher, A. Regulation of actin-based apical structures on epithelial cells. J. Cell Sci. 131, (2018) doi: 10.1242/jcs.221853.

368. Bystriansky, J. S. \& Kaplan, J. H. Sodium pump localization in epithelia. $J$. Bioenerg. Biomembr. 39, 373-378 (2007) doi: 10.1007/s10863-007-9100-3.

369. Inman, G. J. et al. SB-431542 is a potent and specific inhibitor of transforming growth factor-beta superfamily type I activin receptor-like kinase (ALK) receptors ALK4, ALK5, and ALK7. Mol. Pharmacol. 62, 65-74 (2002) doi: $\underline{10.1124 / \mathrm{mol} .62 .1 .65}$.

370. Moser, L. A. \& Schultz-Cherry, S. Suppression of astrovirus replication by an ERK1/2 inhibitor. J. Virol. 82, 7475-7482 (2008) doi: 10.1128/JVI.02193-07.

371. Arango Duque, G. \& Acevedo Ospina, H. A. Understanding TGEV-ETEC Coinfection through the Lens of Proteomics: A Tale of Porcine Diarrhea. Proteomics Clin. Appl. 12, (2018) doi: 10.1002/prca.201700143.

372. Xia, L., Dai, L., Zhu, L., Hu, W. \& Yang, Q. Proteomic Analysis of IPEC-J2 Cells in Response to Coinfection by Porcine Transmissible Gastroenteritis Virus and Enterotoxigenic Escherichia coli K88. PROTEOMICS - Clin. Appl. 11, 1600137 (2017) doi: 10.1002/prca.201600137.

373. Cortez, V. et al. Astrovirus Biology and Pathogenesis. Annu. Rev. Virol. (2017) doi:10.1146/annurev-virology-101416-041742.

374. Guix, S., Caballero, S., Bosch, A. \& Pintó, R. M. Human astrovirus C-terminal nsP1a protein is involved in RNA replication. Virology 333, 124-131 (2005) doi: 10.1016/j.virol.2004.12.023. 
375. Guix, S., Caballero, S., Fuentes, C., Bosch, A. \& Pintó, R. M. Genetic analysis of the hypervariable region of the human astrovirus nsP 1 a coding region: design of a new RFLP typing method. J. Med. Virol. 80, 306-315 (2008) doi: 10.1002/jmv.21058.

376. Zha, Y., Yao, Q., Liu, J.-S., Wang, Y.-Y. \& Sun, W.-M. Hepatitis B virus X protein promotes epithelial-mesenchymal transition and metastasis in hepatocellular carcinoma cell line HCCLM3 by targeting HMGA2. Oncol. Lett. 16, 5709-5714 (2018) doi: 10.3892/ol.2018.9359.

377. Jin, Y. et al. Hepatitis B virus $\mathrm{x}$ protein induces epithelial-mesenchymal transition of hepatocellular carcinoma cells by regulating long non-coding RNA. Virol. $J$. 14, 238 (2017) doi: 10.1186/s12985-017-0903-5.

378. Li, M. et al. Hepatitis B virus X protein promotes renal epithelial-mesenchymal transition in human renal proximal tubule epithelial cells through the activation of NF-kB. Int. J. Mol. Med. 38, 513-520 (2016) doi: 10.3892/ijmm.2016.2637.

379. Hu, B. et al. Hepatitis C virus NS4B protein induces epithelial-mesenchymal transition by upregulation of Snail. Virol. J. 14, 83 (2017) doi: 10.1186/s12985$\underline{017-0737-1 .}$

380. Tiwari, I., Yoon, M.-H., Park, B.-J. \& Jang, K. L. Hepatitis C virus core protein induces epithelial-mesenchymal transition in human hepatocytes by upregulating E12/E47 levels. Cancer Lett. 362, 131-138 (2015) doi: 10.1016/j.canlet.2015.03.032.

381. Zhou, J.-J. et al. Hepatitis C virus core protein increases Snail expression and induces epithelial-mesenchymal transition through the signal transducer and activator of transcription 3 pathway in hepatoma cells. Hepatol. Res. Off. J. Jpn. Soc. Hepatol. 47, 574-583 (2017) doi: 10.1111/hepr.12771.

382. Liu, D. et al. Downregulation of miRNA-30c and miR-203a is associated with hepatitis $\mathrm{C}$ virus core protein-induced epithelial-mesenchymal transition in normal hepatocytes and hepatocellular carcinoma cells. Biochem. Biophys. Res. Commun. 464, 1215-1221 (2015) doi: 10.1016/j.bbrc.2015.07.107.

383. Ranieri, D., Belleudi, F., Magenta, A. \& Torrisi, M. R. HPV16 E5 expression induces switching from FGFR2b to FGFR2 $c$ and epithelial-mesenchymal transition. Int. J. Cancer 137, 61-72 (2015) doi: 10.1002/ijc.29373.

384. Zhang, W. et al. Overexpression of Human Papillomavirus Type 16 Oncoproteins Enhances Epithelial-Mesenchymal Transition via STAT3 Signaling Pathway in Non-Small Cell Lung Cancer Cells. Oncol. Res. 25, 843-852 (2017) doi: $\underline{10.3727 / 096504016 \times 14813880882288 .}$. 
385. Jung, Y.-S., Kato, I. \& Kim, H.-R. C. A novel function of HPV16-E6/E7 in epithelial-mesenchymal transition. Biochem. Biophys. Res. Commun. 435, 339344 (2013) doi: 10.1016/j.bbrc.2013.04.060.

386. Gaur, N., Gandhi, J., Robertson, E. S., Verma, S. C. \& Kaul, R. Epstein-Barr virus latent antigens EBNA3C and EBNA1 modulate epithelial to mesenchymal transition of cancer cells associated with tumor metastasis. Tumour Biol. J. Int. Soc. Oncodevelopmental Biol. Med. 36, 3051-3060 (2015) doi: 10.1007/s13277014-2941-6.

387. Morris, M. A. et al. The EBV-Encoded Oncoprotein, LMP1, Induces an Epithelial-to-Mesenchymal Transition (EMT) via Its CTAR1 Domain through Integrin-Mediated ERK-MAPK Signalling. Cancers 10, (2018) doi: $\underline{10.3390 / \text { cancers } 10050130 .}$.

388. Kim, S.-M. et al. Epstein-Barr virus-encoded latent membrane protein 1 induces epithelial to mesenchymal transition by inducing V-set Ig domain containing 4 (VSIG4) expression via NF-kB in renal tubular epithelial HK-2 cells. Biochem. Biophys. Res. Commun. 492, 316-322 (2017) doi: 10.1016/j.bbrc.2017.08.116.

389. Wasil, L. R. \& Shair, K. H. Y. Epstein-Barr virus LMP1 induces focal adhesions and epithelial cell migration through effects on integrin- $\alpha 5$ and N-cadherin. Oncogenesis 4, e171 (2015) doi: 10.1038/oncsis.2015.31.

390. Ma, L., Teruya-Feldstein, J. \& Weinberg, R. A. Tumour invasion and metastasis initiated by microRNA-10b in breast cancer. Nature 449, 682-688 (2007) doi: $\underline{10.1038 / \text { nature } 06174 .}$.

391. Cardin, S.-E. \& Borchert, G. M. Viral MicroRNAs, Host MicroRNAs Regulating Viruses, and Bacterial MicroRNA-Like RNAs. in Bioinformatics in MicroRNA Research (eds. Huang, J. et al.) 39-56 (Springer, 2017). doi:10.1007/978-1-49397046-9 3.

392. Lin, C. et al. EBV-miR-BART8-3p induces epithelial-mesenchymal transition and promotes metastasis of nasopharyngeal carcinoma cells through activating NF-kB and Erk1/2 pathways. J. Exp. Clin. Cancer Res. CR 37, 283 (2018) doi: 10.1186/s13046-018-0953-6.

393. Yan, Q. et al. EBV-miR-BART10-3p facilitates epithelial-mesenchymal transition and promotes metastasis of nasopharyngeal carcinoma by targeting BTRC. Oncotarget 6, 41766-41782 (2015) doi: 10.18632/oncotarget.6155.

394. Tange, S., Zhou, Y., Nagakui-Noguchi, Y., Imai, T. \& Nakanishi, A. Initiation of human astrovirus type 1 infection was blocked by inhibitors of phosphoinositide 3-kinase. Virol. J. 10, 153 (2013) doi: 10.1186/1743-422X-10-153. 
395. Xu, W., Yang, Z. \& Lu, N. A new role for the PI3K/Akt signaling pathway in the epithelial-mesenchymal transition. Cell Adhes. Migr. 9, 317-324 (2015) doi: 10.1080/19336918.2015.1016686.

396. McCartney-Francis, N. L., Frazier-Jessen, M. \& Wahl, S. M. TGF- $\beta$ : A Balancing Act. Int. Rev. Immunol. 16, 553-580 (1998) doi: 10.3109/08830189809043009.

397. World Health Organization. Diarrhoeal disease fact sheet. (2017) https://www.who.int/news-room/fact-sheets/detail/diarrhoeal-disease.

398. Lin, H.-C. et al. Astrovirus Gastroenteritis in Children in Taipei. J. Formos. Med. Assoc. 107, 295-303 (2008) doi: 10.1016/S0929-6646(08)60090-X.

399. Tseng, W.-C. et al. Astrovirus gastroenteritis in hospitalized children of less than 5 years of age in Taiwan, 2009. J. Microbiol. Immunol. Infect. 45, 311-317 (2012) doi: 10.1016/j.jmii.2011.12.017.

400. Amaral, M. S. C. et al. The prevalence of norovirus, astrovirus and adenovirus infections among hospitalised children with acute gastroenteritis in Porto Velho, state of Rondônia, western Brazilian Amazon. Mem. Inst. Oswaldo Cruz 110, 215-221 (2015) doi: 10.1590/0074-02760140381.

401. Cordey, S., Zanella, M.-C., Wagner, N., Turin, L. \& Kaiser, L. Novel human astroviruses in pediatric respiratory samples: A one-year survey in a Swiss tertiary care hospital. J. Med. Virol. 90, 1775-1778 (2018) doi: 10.1002/jmv.25246.

402. Trabattoni, D. et al. Thiazolides Elicit Anti-Viral Innate Immunity and Reduce HIV Replication. Sci. Rep. 6, 1-10 (2016) doi: 10.1038/srep27148.

403. Hong, S. K. et al. Nitazoxanide suppresses IL-6 production in LPS-stimulated mouse macrophages and TG-injected mice. Int. Immunopharmacol. 13, 23-27 (2012) doi: 10.1016/j.intimp.2012.03.002.

404. Shi, Z. et al. Nitazoxanide inhibits the replication of Japanese encephalitis virus in cultured cells and in a mouse model. Virol. J. 11, 10 (2014) doi: $\underline{10.1186 / 1743-}$ 422X-11-10.

405. El-Kowrany, S. I., El Ghaffar, A. E.-S. A., Shoheib, Z. S., Mady, R. F. \& Gamea, G. A. M. Evaluation of nitazoxanide as a novel drug for the treatment of acute and chronic toxoplasmosis. Acta Trop. 195, 145-154 (2019) doi: 10.1016/j.actatropica.2019.04.013.

406. Rossignol, J.-F. Nitazoxanide: A first-in-class broad-spectrum antiviral agent. Antiviral Res. 110, 94-103 (2014) doi: 10.1016/j.antiviral.2014.07.014. 
407. Rossignol, J. F., La Frazia, S., Chiappa, L., Ciucci, A. \& Santoro, M. G. Thiazolides, a new class of anti-influenza molecules targeting viral hemagglutinin at the post-translational level. J. Biol. Chem. 284, 29798-29808 (2009) doi: 10.1074/jbc.M109.029470.

408. Rossignol, J.-F., Abu-Zekry, M., Hussein, A. \& Santoro, M. G. Effect of nitazoxanide for treatment of severe rotavirus diarrhoea: randomised double-blind placebo-controlled trial. Lancet Lond. Engl. 368, 124-129 (2006) doi: 10.1016/S0140-6736(06)68852-1.

409. Rossignol, J.-F. \& El-Gohary, Y. M. Nitazoxanide in the treatment of viral gastroenteritis: a randomized double-blind placebo-controlled clinical trial. Aliment. Pharmacol. Ther. 24, 1423-1430 (2006) doi: 10.1111/j.13652036.2006.03128.x.

410. Perelygina, L. et al. Inhibition of rubella virus replication by the broad-spectrum drug nitazoxanide in cell culture and in a patient with a primary immune deficiency. Antiviral Res. 147, 58-66 (2017) doi: 10.1016/j.antiviral.2017.09.019.

411. Li, Z. et al. Existing drugs as broad-spectrum and potent inhibitors for Zika virus by targeting NS2B-NS3 interaction. Cell Res. 27, 1046-1064 (2017) doi: 10.1038/cr.2017.88.

412. Rossignol, J., Elfert, A., El-Gohary, Y. \& Keeffe, E. B. Improved Virologic Response in Chronic Hepatitis C Genotype 4 Treated With Nitazoxanide, Peginterferon, and Ribavirin. Gastroenterology 136, 856-862 (2009) doi: 10.1053/j.gastro.2008.11.037.

413. Korba, B. E. et al. Nitazoxanide, tizoxanide and other thiazolides are potent inhibitors of hepatitis B virus and hepatitis C virus replication. Antiviral Res. 77, 56-63 (2008) doi: 10.1016/j.antiviral.2007.08.005.

414. Haffizulla, J. et al. Effect of nitazoxanide in adults and adolescents with acute uncomplicated influenza: a double-blind, randomised, placebo-controlled, phase 2b/3 trial. Lancet Infect. Dis. 14, 609-618 (2014) doi: 10.1016/S1473$\underline{3099(14) 70717-0 .}$.

415. Teran, C. G., Teran-Escalera, C. N. \& Villarroel, P. Nitazoxanide vs. probiotics for the treatment of acute rotavirus diarrhea in children: a randomized, singleblind, controlled trial in Bolivian children. Int. J. Infect. Dis. 13, 518-523 (2009) doi: $10.1016 / \mathrm{j} . \mathrm{ijid} .2008 .09 .014$.

416. Siddiq, D. M., Koo, H. L., Adachi, J. A. \& Viola, G. M. Norovirus gastroenteritis successfully treated with nitazoxanide. J. Infect. 63, 394-397 (2011) doi: 10.1016/j.jinf.2011.08.002. 
417. Rossignol, J.-F. \& Keeffe, E. B. Thiazolides: a new class of drugs for the treatment of chronic hepatitis B and C. Future Microbiol. 3, 539-545 (2008) doi: $\underline{10.2217 / 17460913.3 .5 .539 .}$.

418. Rossignol, J.-F., Elfert, A. \& Keeffe, E. B. Treatment of chronic hepatitis C using a 4-week lead-in with nitazoxanide before peginterferon plus nitazoxanide. $J$. Clin. Gastroenterol. 44, 504-509 (2010) doi: 10.1097/MCG.0b013e3181bf9b15.

419. Hoffman, P. S. et al. Antiparasitic drug nitazoxanide inhibits the pyruvate oxidoreductases of Helicobacter pylori, selected anaerobic bacteria and parasites, and Campylobacter jejuni. Antimicrob. Agents Chemother. 51, 868-876 (2007) doi: 10.1128/AAC.01159-06.

420. Caballero, S. et al. Persistent gastroenteritis in children infected with astrovirus: association with serotype-3 strains. J. Med. Virol. 71, 245-250 (2003) doi: $\underline{10.1002 / j m v .10476 .}$.

421. Yoshimatsu, Y. et al. TNF- $\alpha$ enhances TGF- $\beta$-induced endothelial-tomesenchymal transition via TGF- $\beta$ signal augmentation. Cancer Sci. (2020) doi: $10.1111 /$ cas. 14455 .

422. Shrestha, R., Bridle, K. R., Crawford, D. H. G. \& Jayachandran, A. TNF- $\alpha$-mediated epithelial-to-mesenchymal transition regulates expression of immune checkpoint molecules in hepatocellular carcinoma. Mol. Med. Rep. 21, 1849-1860 (2020) doi: 10.3892/mmr.2020.10991.

423. Liu, W. et al. IL-6 promotes metastasis of non-small-cell lung cancer by upregulating TIM-4 via NF-кB. Cell Prolif. 53, e12776 (2020) doi: $\underline{10.1111 / \text { cpr.12776. }}$.

424. Annes, J. P. Making sense of latent TGFbeta activation. J. Cell Sci. 116, 217-224 (2003) doi: $10.1242 /$ jcs.00229.

425. Ma, M. et al. MiR-487a Promotes TGF- $\beta 1$-induced EMT, the Migration and Invasion of Breast Cancer Cells by Directly Targeting MAGI2. Int. J. Biol. Sci. 12, 397-408 (2016) doi: 10.7150/ijbs.13475.

426. Rao, R. Occludin phosphorylation in regulation of epithelial tight junctions. Ann. N. Y. Acad. Sci. 1165, 62-68 (2009) doi: 10.1111/j.1749-6632.2009.04054.x.

427. Elias, B. C. et al. Phosphorylation of Tyr-398 and Tyr-402 in occludin prevents its interaction with ZO-1 and destabilizes its assembly at the tight junctions. $J$. Biol. Chem. 284, 1559-1569 (2009) doi: 10.1074/jbc.M804783200. 
428. Manda, B. et al. Phosphorylation hotspot in the C-terminal domain of occludin regulates the dynamics of epithelial junctional complexes. J. Cell Sci. 131, (2018) doi: $10.1242 /$ jcs. 206789 . 


\section{APPENDIX A. PRO-INFLAMMATORY CYTOKINES ARE NOT RESPONSIBLE FOR HASTV-INDUCED EMT}

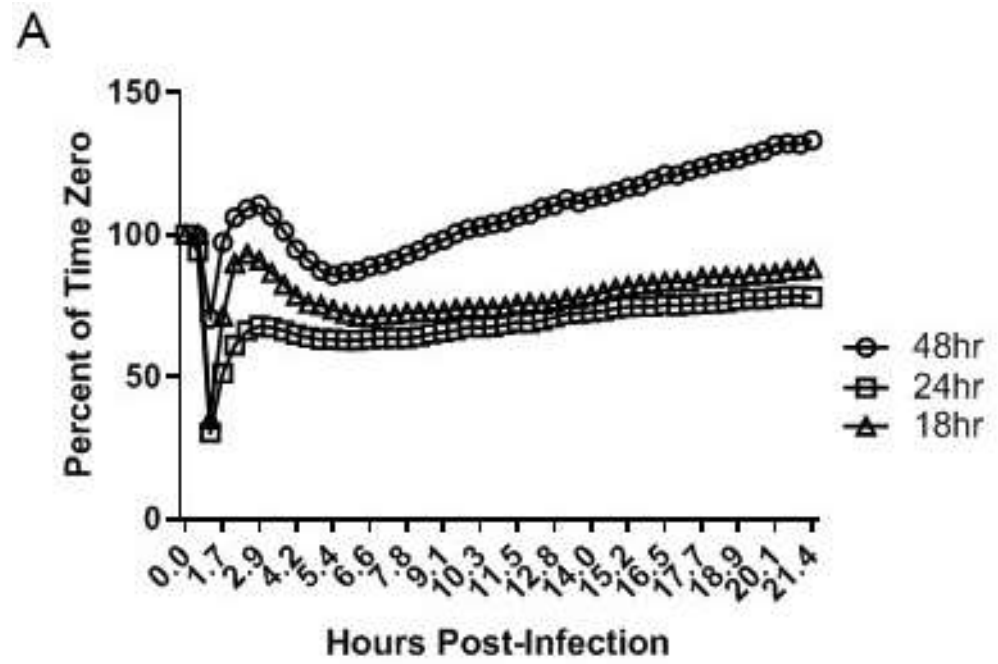

B
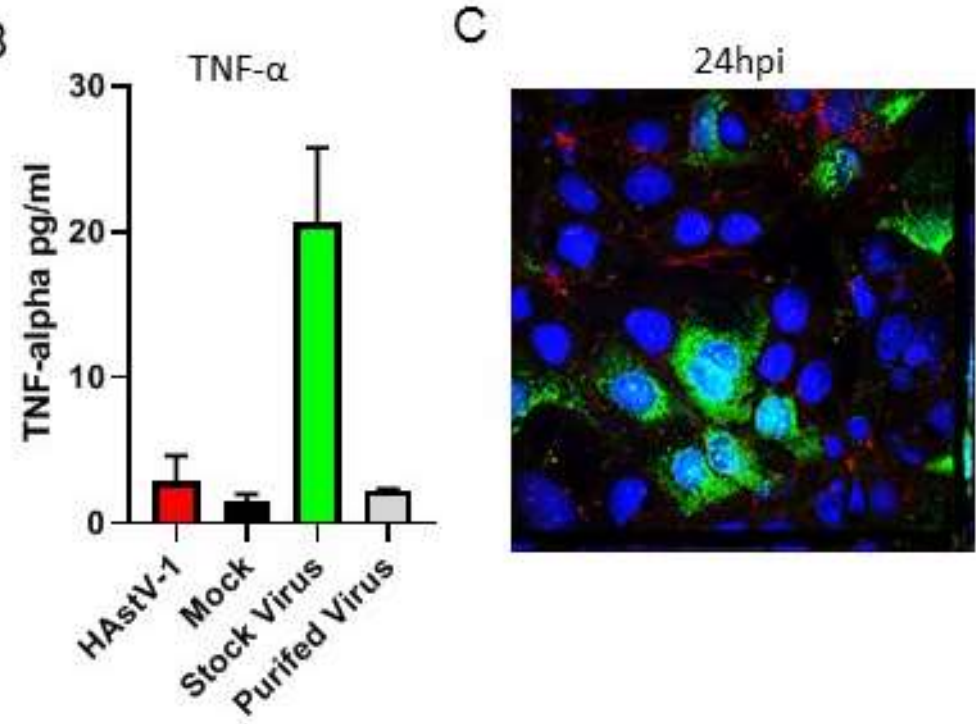

Figure A-1. Pro-Inflammatory Cytokines Are Not Responsible for HAstV-Induced EMT.

(A) Supernatants collected from HAstV-1 infected Caco-2 cells at 18, 24, and 48 hpi were UV-inactivated and place onto naïve Caco-2 cells. TER was measured every 15 min for 21 hours following inoculation with UV supernatants. (B) TNF- $\alpha$ levels were measured in supernatants from HAstV-1 and mock infected cells (24hpi0, as well as stock virus and purified virus. (C) Caco-2 cells were infected with purified HAstV-1 (MOI 10) and stained for HAstV capsid (green), E-cadherin (red), and DAPI (blue) at 24 hpi. 


\section{APPENDIX B. HASTV DIRECTLY ACTIVATES LATENT TGF-B}

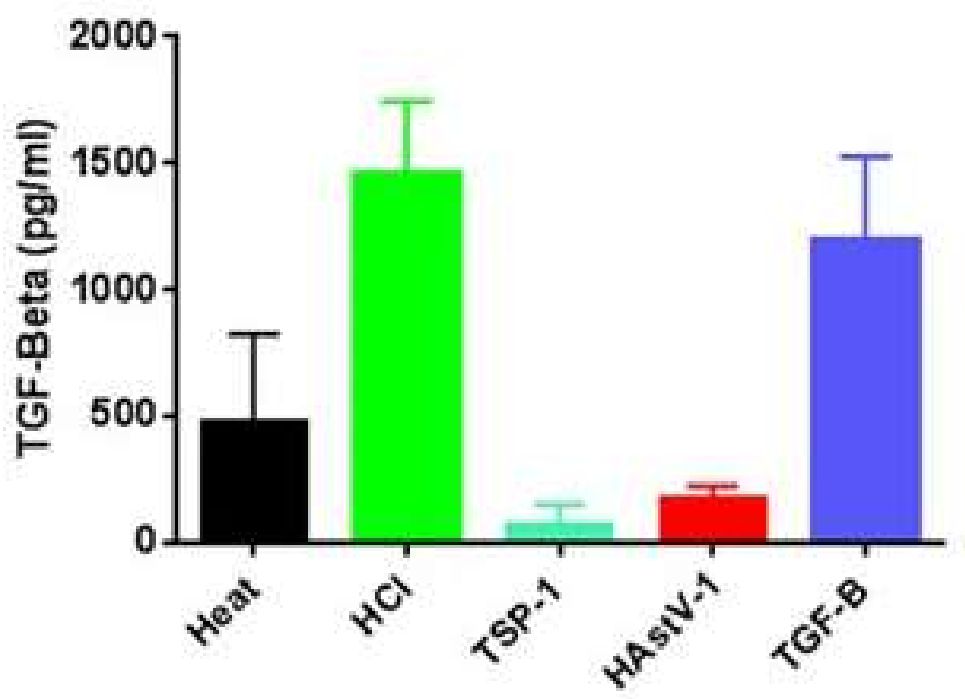

Figure B-1. HAstV Directly Activates Latent TGF- $\beta$.

(A) Conditioned media containing latent TGF- $\beta$ from Caco- 2 cells was treated with heat $\left(100^{\circ} \mathrm{C}\right.$ for $10 \mathrm{~min}$ ), $1 \mathrm{~N} \mathrm{HCl}$, thrombospondin-1 (TSP-1; $4 \mu \mathrm{g} / \mathrm{ml}$ ), or purified HAstV-1 ( $10 \%$ by volume). Active TGF- $\beta$ was included as a positive control. Samples were then assayed for active TGF- $\beta$ using the PAI assay as described previously. ${ }^{344}$ Error bars indicate standard deviations. 


\section{APPENDIX C. CLINICAL ISOLATE WITHOUT SEQUENCE HOMOLOGY TO MIR-487A SHOWS REDUCED EMT CHARACTERISTICS}

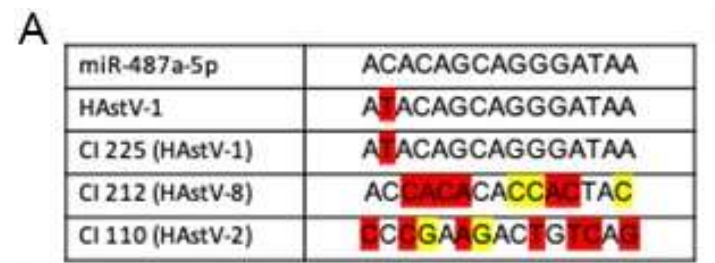

B

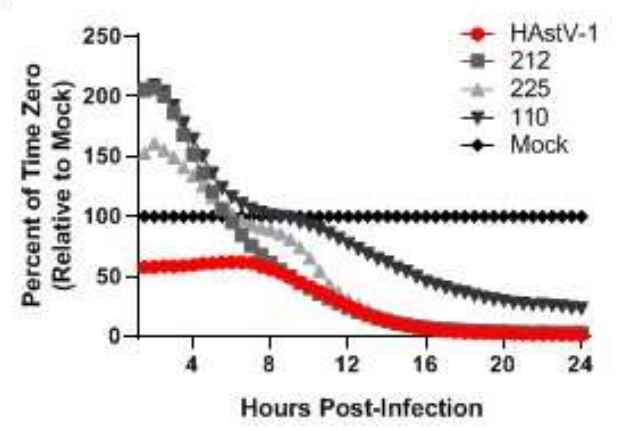

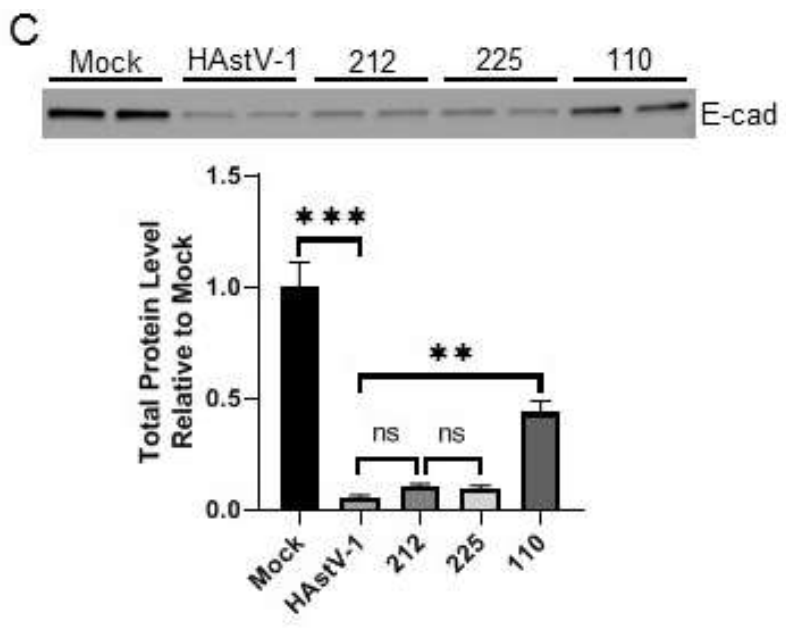

Figure C-1. Clinical Isolate Without Sequence Homology to miR-487a Shows Reduced EMT Characteristics.

(A) Sequence homology of lab adapted HAstV-1, and clinical isolates 225, 212, and 110 to miR-487a. Highlighted in red are unacceptable substitutions and yellow are acceptable substitutions. (B) TER measurements from 0 to $24 \mathrm{hpi}$ with lab adapted HAstV-1 and clinical isolates 225, 212, and 110. (C) Expression of epithelial marker, E-cadherin, was quantified by immunoblot of HAstV-1, clinical isolates 225, 212, and 110, or mock infected Caco- 2 cell lysates. Bands were then quantified by densitometry and normalized to total protein then compared to mock-infection. Error bars indicate standard deviations, and asterisks show statistical significance as measured by two-way ANOVA followed by Tukey's multiple comparisons test as follows: *, $\mathrm{P}<0.05$; **, $\mathrm{P}<0.01$; ***, $\mathrm{P}<0.001$. 


\section{APPENDIX D. THE ROLE OF OCCLUDIN IN HASTV INFECTION}

A

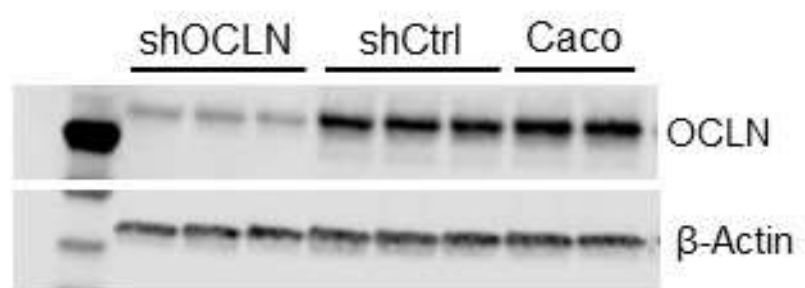

B

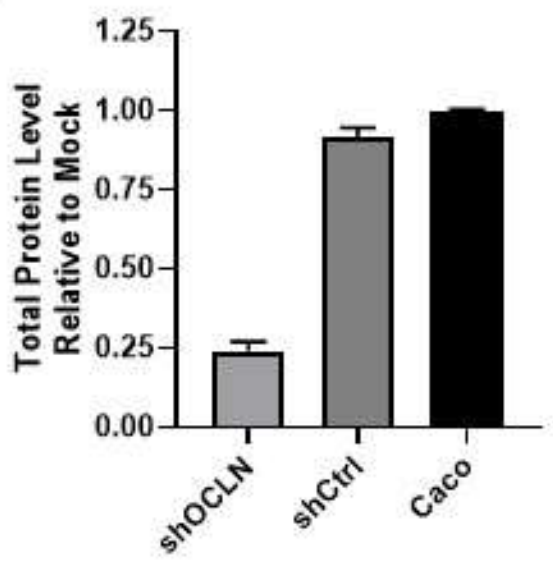

Figure D-1. Occludin Knockdown Caco-2 Cells.

Caco-2 cells stably transfected with shRNA directed to occludin show decreased expression of occludin as compared to scramble shRNA (shCtrl) and wildtype Caco-2 cells. (A) Expression of occludin was quantified by immunoblot of shOCLN, shCtrl, and Caco- 2 cell lysates. (B) Bands were then quantified by densitometry and normalized to $\beta$ actin. Error bars indicate standard deviations. 
A

shCtrl
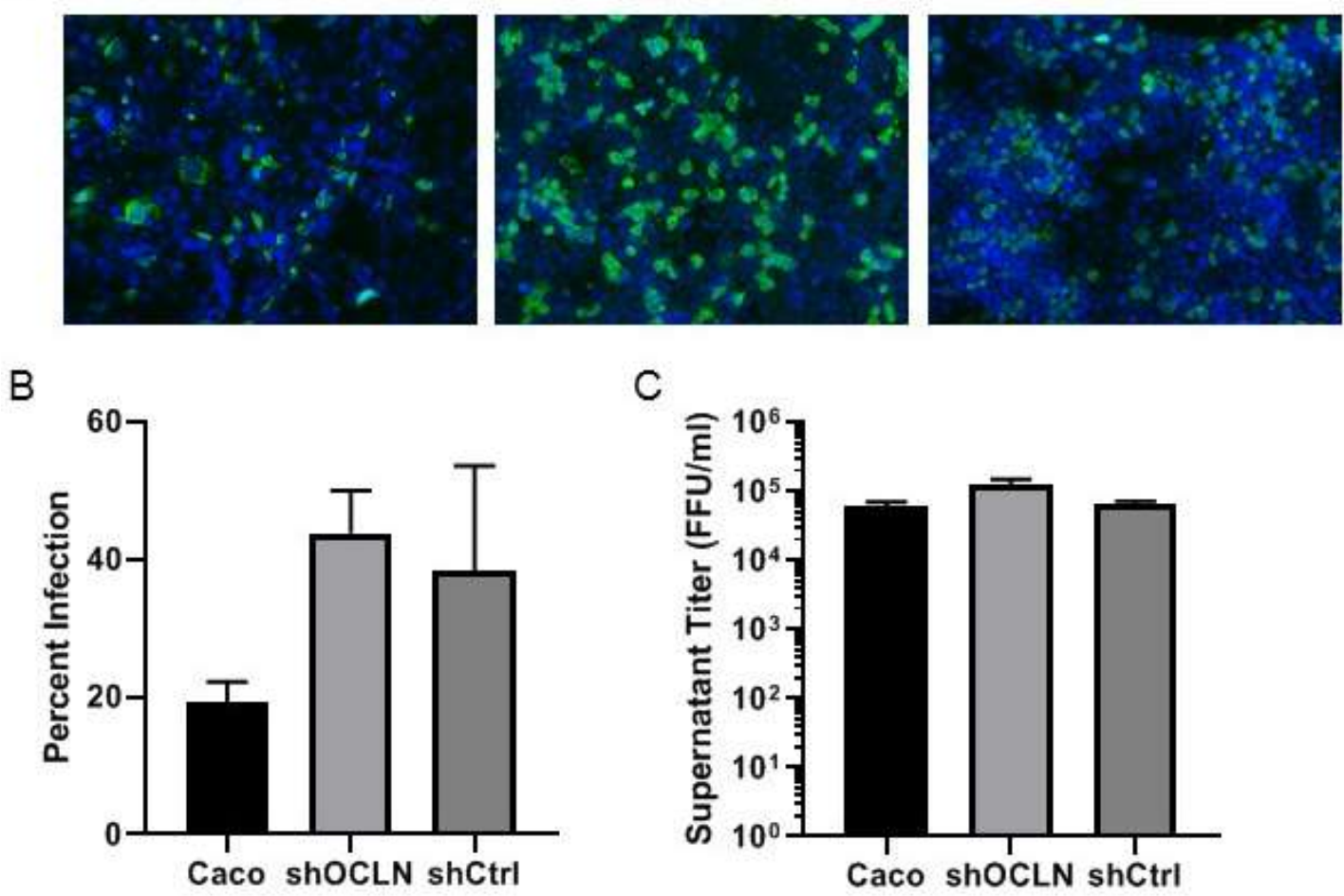

C

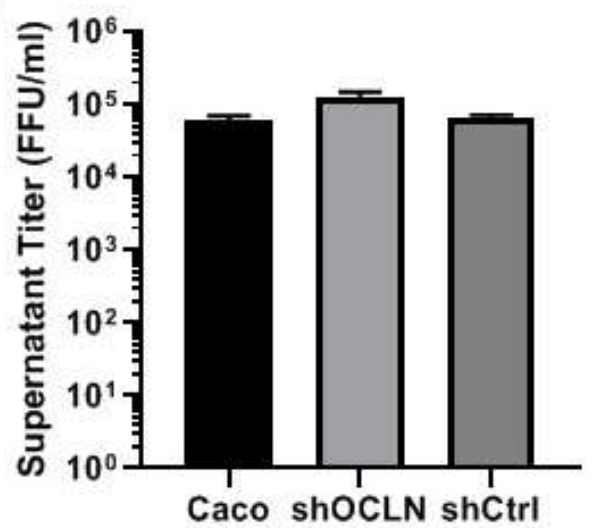

Figure D-2. Occludin Knockdown Cells Exhibit Increased Infection.

(A) Caco-2, shOCLN, and shCtrl cells were infected with HAstV-1 (MOI 5). At 24hpi, the cells were fixed in $100 \%$ ice-cold methanol and stained for HAstV capsid (green) and DAPI (blue). (B) The percentage of infected cells was quantified for each cell type. (C) Supernatants collected at $24 \mathrm{hpi}$ from HAstV-1-infected Caco-2, shOCLN, and shCtrl cells were titered, showing no significant difference between the cell types. 


\section{APPENDIX E. HASTV-VA1 ANTIBODIES ARE MORE ABUNDANT THAN HASTV-1}

A

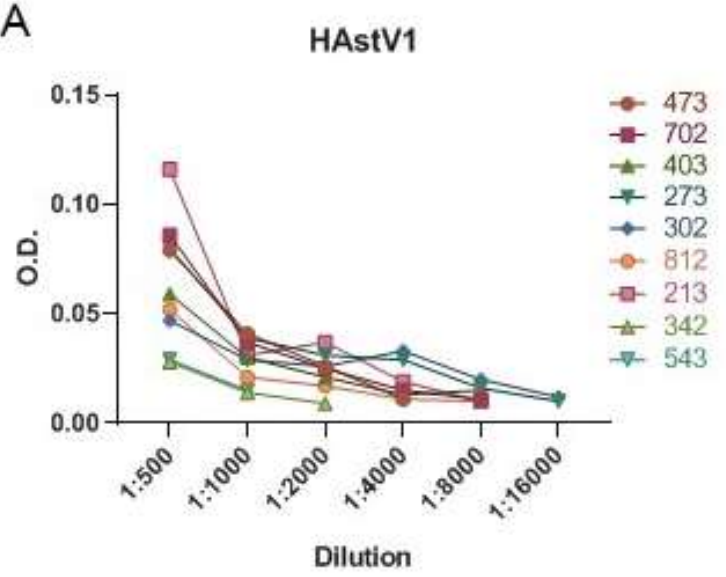

C

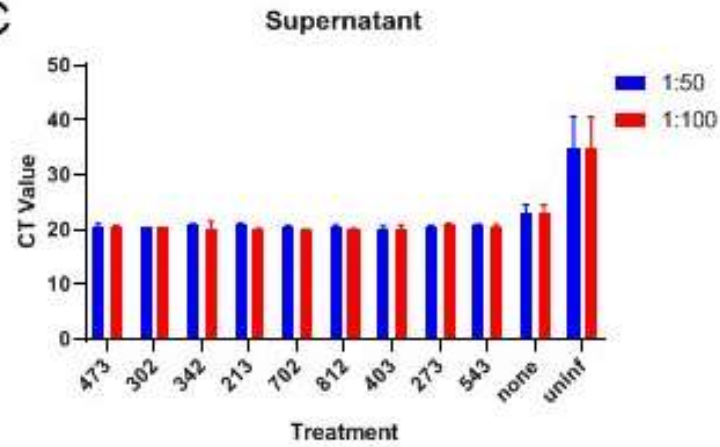

B

VA1

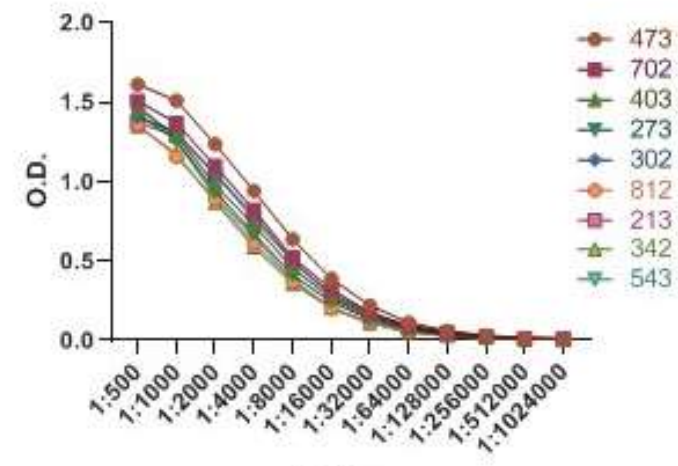

Dilution

D

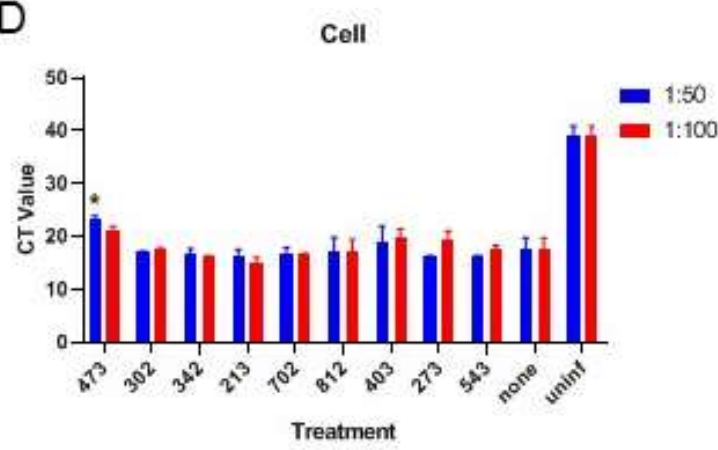

Figure E-1. HAstV-VA1 Antibodies Are More Abundant than HAstV-1. Nine lots of commercially available IVIG were tested by ELISA for specificity to HAstV-1 (A) and HAstV-VA1 (B). Viral titers were analyzed via RT-PCR following neutralization assay in the supernatant (C) and cell lysate (D) of HAstV-VA1 infected cells. 


\section{VITA}

Virginia Hargest was born in Charleston, South Carolina in 1992. She obtained Bachelor of Science degree with an emphasis in Microbiology with honors in May 2014 from the University of Tennessee, Knoxville. She matriculated into the Integrated Program in Biomedical Science (IPBS), the Microbiology, Immunology, and Biochemistry track for doctoral studies at University of Tennessee Health Science Center, Memphis, TN, USA in August 2015. She joined the Schultz-Cherry Lab at St. Jude Children's Research Hospital in January of 2016. She received her doctoral degree in June 2020. 